\title{
Multidimensional Access Methods
}

\author{
VOLKER GAEDE \\ IC-Parc, Imperial College, London
}

AND
OLIVER GÜNTHER

Humboldt-Universität, Berlin

\begin{abstract}
Search operations in databases require special support at the physical level. This is true for conventional databases as well as spatial databases, where typical search operations include the point query (find all objects that contain a given search point) and the region query (find all objects that overlap a given search region). More than ten years of spatial database research have resulted in a great variety of multidimensional access methods to support such operations. We give an overview of that work. After a brief survey of spatial data management in general, we first present the class of point access methods, which are used to search sets of points in two or more dimensions. The second part of the paper is devoted to spatial access methods to handle extended objects, such as rectangles or polyhedra. We conclude with a discussion of theoretical and experimental results concerning the relative performance of various approaches.
\end{abstract}

Categories and Subject Descriptors: H.2.2 [Database Management]: Physical Design-access methods; H.2.4 [Database Management]: Systems; H.2.8

[Database Management]: Database Applications-spatial databases and GIS; H.3.3 [Information Storage and Retrieval]: Information Search and Retrievalsearch process, selection process

General Terms: Design, Experimentation, Performance

Additional Key Words and Phrases: Data structures, multidimensional access methods

\section{INTRODUCTION}

With an increasing number of computer applications that rely heavily on multidimensional data, the database community has recently devoted considerable attention to spatial data management. Although the main motivation origi- nated in the geosciences and mechanical CAD, the range of possible applications has expanded to areas such as robotics, visual perception, autonomous navigation, environmental protection, and medical imaging [Günther and Buchmann 1990].

The range of interpretation given to

This work was partially supported by the German Research Society (DFG/SFB 373) and by the ESPRIT Working Group CONTESSA (8666).

Authors' address: Institut für Wirtschaftsinformatik, Humboldt-Universität zu Berlin, Spandauer Str. 1, 10178 Berlin, Germany; email: 〈gaede,guenther\}@wiwi.hu-berlin.de).

Permission to make digital / hard copy of part or all of this work for personal or classroom use is granted without fee provided that the copies are not made or distributed for profit or commercial advantage, the copyright notice, the title of the publication, and its date appear, and notice is given that copying is by permission of the ACM, Inc. To copy otherwise, to republish, to post on servers, or to redistribute to lists, requires prior specific permission and/or a fee.

(c) 1998 ACM 0360-0300/98/0600-0170 \$05.00 


\section{CONTENTS}

1. INTRODUCTION

2. ORGANIZATION OF SPATIAL DATA

2.1 What Is Special About Spatial?

2.2 Definitions and Queries

3. BASIC DATA STRUCTURES

3.1 One-Dimensional Access Methods

3.2 Main Memory Structures

4. POINT ACCESS METHODS

4.1 Multidimensional Hashing

4.2 Hierarchical Access Methods

4.3 Space-Filling Curves for Point Data

5. SPATIAL ACCESS METHODS

5.1 Transformation

5.2 Overlapping Regions

5.3 Clipping

5.4 Multiple Layers

6. COMPARATIVE STUDIES

7. CONCLUSIONS

the term spatial data management is just as broad as the range of applications. In VLSI CAD and cartography, this term refers to applications that rely mostly on two-dimensional or layered two-dimensional data. VLSI data are usually represented by rectilinear polylines or polygons whose edges are isooriented, that is, parallel to the coordinate axes. Typical operations include intersection and geometric routing [Shekhar and Liu 1995]. Cartographic data are also two-dimensional with points, lines, and regions as basic primitives. In contrast to VLSI CAD, however, the shapes are often characterized by extreme irregularities. Common operations include spatial searches and map overlay, as well as distance-related operations. In mechanical CAD, on the other hand, data objects are usually three-dimensional solids. They may be represented in a variety of data formats, including cell decomposition schemes, constructive solid geometry (CSG), and boundary representations [Kemper and Wallrath 1987]. Yet other applications emphasize the processing of unanalyzed images, such as X-rays and satellite imagery, from which features are extracted. In those areas, the terms spa- tial database and image database are sometimes even used interchangeably.

Strictly speaking, however, spatial databases contain multidimensional data with explicit knowledge about objects, their extent, and their position in space. The objects are usually represented in some vector-based format, and their relative positions may be explicit or implicit (i.e., derivable from the internal representation of their absolute positions). I mage databases often place less emphasis on data analysis. They provide storage and retrieval for unanalyzed pictorial data, which are typically represented in some raster format. Techniques developed for the storage and manipulation of image data can be applied to other media as well, such as infrared sensor signals or sound.

In this survey we assume that the goal is to manipulate analyzed multidimensional data and that unanalyzed images are handled only as the source from which spatial data can be derived. The challenge for the developers of a spatial database system lies not so much in providing yet another collection of special-purpose data structures. Rather, one has to find abstractions and architectures to implement generic systems, that is, to build systems with generic spatial data-management capabilities that can be tailored to the requirements of a particular application domain. Important issues in this context include the handling of spatial representations and data models, multidimensional access methods, and pictorial or spatial query languages and their optimization.

This article is a survey of multidimensional access methods to support search operations in spatial databases. Figure 1, which was inspired by a similar graph by Lu and Ooi [1993], gives a first overview of the diversity of existing multidimensional access methods. The goal is not to describe all of these structures, but to discuss the most prominent ones, to present possible taxonomies, and to establish references to other literature. 


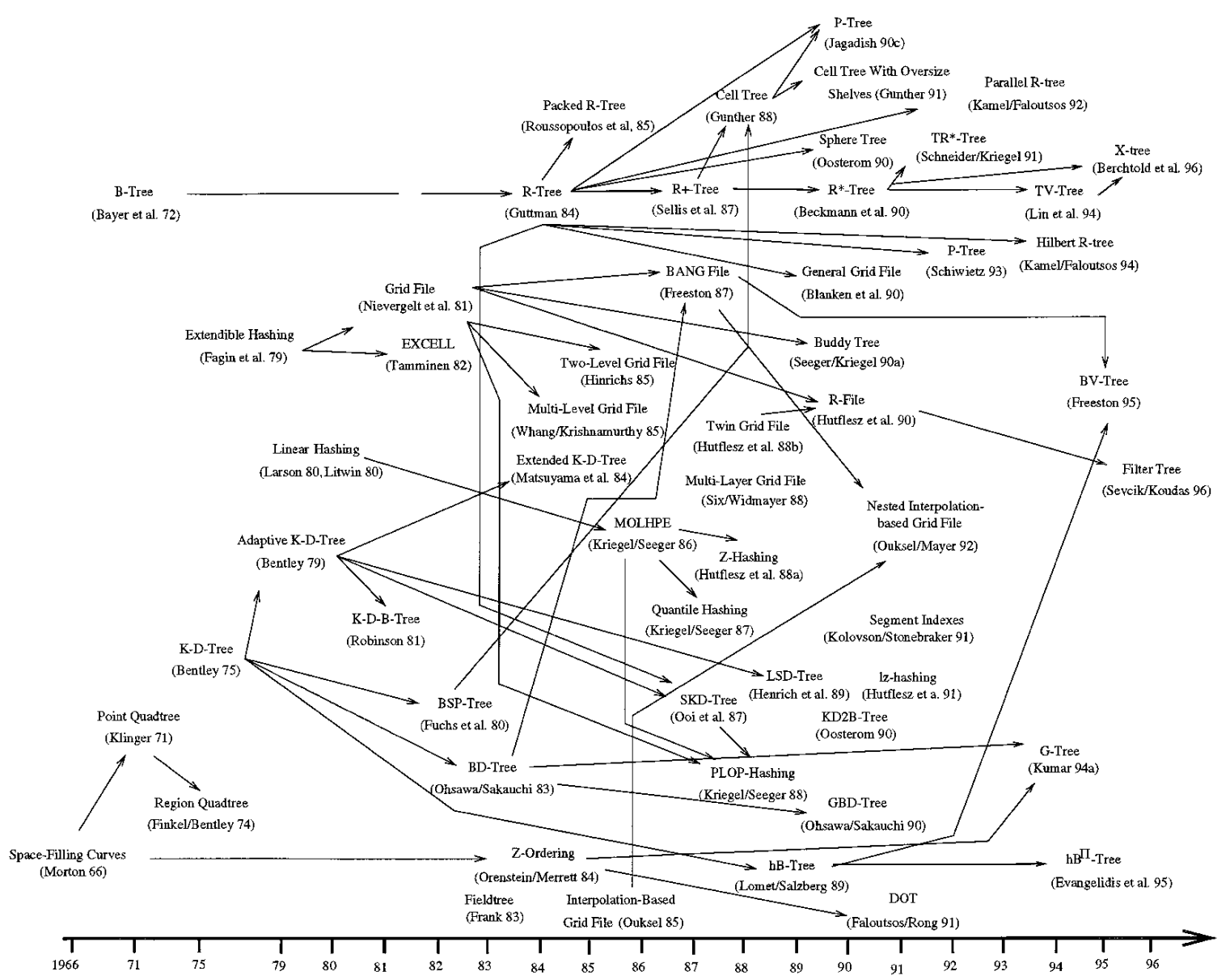

Figure 1. History of multidimensional access methods.

Several shorter surveys have been published previously in various Ph.D. theses such as Ooi [1990], Kolovson [1990], Oosterom [1990], and Schiwietz [1993]. Widmayer [1991] gives an overview of work published before 1991. Like the thesis by Schiwietz, however, his survey is available only in German. Samet's books [1989, 1990] present the state of the art until 1989. However, they primarily cover quadtrees and related data structures. Lomet [1991] discusses the field from a systems-oriented point of view.

The remainder of the article is organized as follows. Section 2 discusses some basic properties of spatial data and their implications for the design and implementation of spatial databases. Section 3 gives an overview of some traditional data structures that had an impact on the design of multidimensional access methods. Sections 4 and 5 form the core of this survey, presenting a variety of point access methods (PAMs) and spatial access methods (SAMs), respectively. Some remarks about theoretical and experimental analyses are contained in Section 6, and Section 7 concludes the article.

\section{ORGANIZATION OF SPATIAL DATA}

\subsection{What Is Special About Spatial?}

To obtain a better understanding of the requirements in spatial database systems, we first discuss some basic properties of spatial data. First, spatial data have a complex structure. A spatial data object may be composed of a single point or several thousands of polygons, 
arbitrarily distributed across space. It is usually not possible to store collections of such objects in a single relational table with a fixed tuple size. Second, spatial data are often dynamic. Insertions and deletions are interleaved with updates, and data structures used in this context have to support this dynamic behavior without deteriorating over time. Third, spatial databases tend to be large. Geographic maps, for example, typically occupy several gigabytes of storage. The integration of secondary and tertiary memory is therefore essential for efficient processing [Chen et al. 1995]. Fourth, there is no standard algebra defined on spatial data, although several proposals have been made in the past [Egenhofer 1989; Güting 1989; Scholl and Voisard 1989; Güting and Schneider 1993]. This means in particular that there is no standardized set of base operators. The set of operators depends heavily on the given application domain, although some operators (such as intersection) are more common than others. Fifth, many spatial operators are not closed. The intersection of two polygons, for example, may return any number of single points, dangling edges, or disjoint polygons. This is particularly relevant when operators are applied consecutively. Sixth, although the computational costs vary among spatial database operators, they are generally more expensive than standard relational operators.

An important class of geometric operators that needs special support at the physical level is the class of spatial search operators. Retrieval and update of spatial data are usually based not only on the value of certain alphanumeric attributes but also on the spatial location of a data object. A retrieval query on a spatial database often requires the fast execution of a geometric search operation such as a point or region query. Both operations require fast access to those data objects in the database that occupy a given location in space.

To support such search operations, one needs special multidimensional access methods. The main problem in the design of such methods, however, is that there exists no total ordering among spatial objects that preserves spatial proximity. In other words, there is no mapping from two- or higher-dimensional space into one-dimensional space such that any two objects that are spatially close in the higher-dimensional space are also close to each other in the one-dimensional sorted sequence.

This makes the design of efficient access methods in the spatial domain much more difficult than in traditional databases, where a broad range of efficient and well-understood access methods is available. Examples for such onedimensional access methods (also called single key structures, although that term is somewhat misleading) include the B-tree [Bayer and McCreight 1972] and extendible hashing [Fagin et al. 1979]; see Section 3.1 for a brief discussion. A popular approach to handling multidimensional search queries consists of the consecutive application of such single key structures, one per dimension. Unfortunately, this approach can be very inefficient [Kriegel 1984]. Since each index is traversed independently of the others, we cannot exploit the possibly high selectivity in one dimension to narrow down the search in the remaining dimensions. In general, there is no easy and obvious way to extend single key structures in order to handle multidimensional data.

There is a variety of requirements that multidimensional access methods should meet, based on the properties of spatial data and their applications [Robinson 1981; Lomet and Salzberg 1989; Nievergelt 1989]:

(1) Dynamics. As data objects are inserted and deleted from the database in any given order, access methods should continuously keep track of the changes.

(2) Secondary/tertiary storage management. Despite growing main memories, it is often not possible to 
hold the complete database in main memory. Therefore, access methods need to integrate secondary and tertiary storage in a seamless manner.

(3) Broad range of supported operations. Access methods should not support just one particular type of operation (such as retrieval) at the expense of other tasks (such as deletion).

(4) Independence of the input data and insertion sequence. Access methods should maintain their efficiency even when input data are highly skewed or the insertion sequence is changed. This point is especially important for data that are distributed differently along the various dimensions.

(5) Simplicity. Intricate access methods with many special cases are often error-prone to implement and thus not sufficiently robust for large-scale applications.

(6) Scalability. Access methods should adapt well to database growth.

(7) Time efficiency. Spatial searches should be fast. A major design goal is to meet the performance characteristics of one-dimensional B-trees: first, access methods should guarantee a logarithmic worst-case search performance for all possible input data distributions regardless of the insertion sequence and second, this worst-case performance should hold for any combination of the $d$ attributes.

(8) Space efficiency. An index should be small in size compared to the data to be addressed and therefore guarantee a certain storage utilization.

(9) Concurrency and recovery. In modern databases where multiple users concurrently update, retrieve, and insert data, access methods should provide robust techniques for transaction management without significant performance penalties.
(10) Minimum impact. The integration of an access method into a database system should have minimum impact on existing parts of the system.

\subsection{Definitions and Queries}

We have already introduced the term multidimensional access methods to denote the large class of access methods that support searches in spatial databases and are the subject of this survey. Within this class, we distinguish between point access methods (PAMs) and spatial access methods (SAMs). Point access methods have primarily been designed to perform spatial searches on point databases (i.e., databases that store only points). The points may be embedded in two or more dimensions, but they do not have a spatial extension. Spatial access methods, however, can manage extended objects, such as lines, polygons, or even higher-dimensional polyhedra. In the literature, one often finds the term spatial access method referring to what we call multidimensional access method. Other terms used for this purpose include spatial index or spatial index structure.

We generally assume that the given objects are embedded in d-dimensional Euclidean space $\mathrm{E}^{\mathrm{d}}$ or a suitable subspace thereof. In this article, this space is also referred to as the universe or original space. Any point object stored in a spatial database has a unique location in the universe, defined by its $d$ coordinates. Unless the distinction is essential, we use the term point both for locations in space and for point objects stored in the database. Note, however, that any point in space can be occupied by several point objects stored in the database.

A (convex) d-dimensional polytope $P$ in $E^{d}$ is defined to be the intersection of some finite number of closed halfspaces in $\mathrm{E}^{\mathrm{d}}$, such that the dimension of the smallest affine subspace containing $P$ is d. If $a \in E^{d}-\{0\}$ and $c \in E^{1}$ then the $(d-1)$-dimensional set $H(a, c)=\{x \in$ 


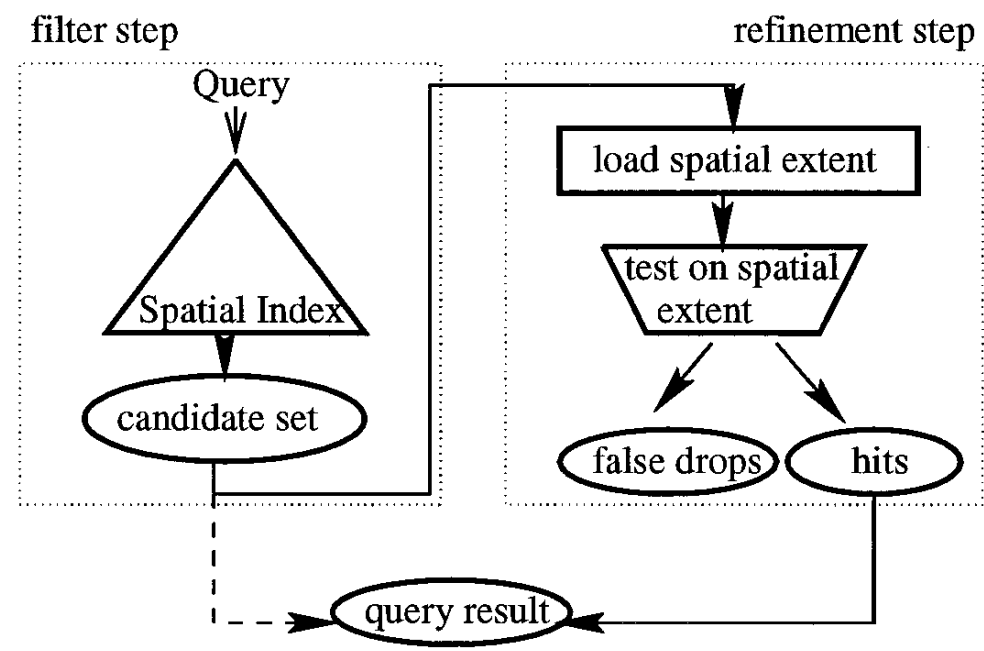

Figure 2. Multistep spatial query processing [Brinkhoff et al. 1994].

$\left.E^{d}: x \cdot a=c\right\}$ defines a hyperplane in $E^{d}$. A hyperplane $\mathrm{H}(\mathrm{a}, \mathrm{c})$ defines two closed halfspaces: the positive halfspace $1 \cdot H(a, c)=\left\{x \in E^{d}: x \cdot a \geq c\right\}$, and the negative halfspace $-1 \cdot H(a, c)=$ $\left\{x \in E^{d}: x \cdot a \leq c\right\}$. A hyperplane $\mathrm{H}(\mathrm{a}, \mathrm{c})$ supports a polytope $\mathrm{P}$ if $\mathrm{H}(\mathrm{a}, \mathrm{c})$ $\cap P \neq \emptyset$ and $P \subseteq 1 \cdot H(a, c)$, that is, if $\mathrm{H}(\mathrm{a}, \mathrm{c})$ embeds parts of P's boundary. If $\mathrm{H}(\mathrm{a}, \mathrm{c})$ is any hyperplane supporting $\mathrm{P}$ then $\mathrm{P} \cap \mathrm{H}(\mathrm{a}, \mathrm{c})$ is a face of $\mathrm{P}$. The faces of dimension 1 are called edges; those of dimension 0 vertices.

By forming the union of some finite number of polytopes $\mathrm{Q}_{1}, \ldots, \mathrm{Q}_{\mathrm{n}}$, we obtain a (d-dimensional) polyhedron Q in $E^{d}$ that is not necessarily convex. Following the intuitive understanding of polyhedra, we require that the $\mathrm{Q}_{\mathrm{i}}(\mathrm{i}=$ $1, \ldots, n)$ be connected. Note that this still allows for polyhedra with holes. Each face of $Q$ is either the face of some $Q_{i}$, or a fraction thereof, or the result of the intersection of two or more $Q_{i}$. Each polyhedron $P$ divides the points in space into three subsets that are mutually disjoint: its interior, its boundary, and its exterior.

Following usual conventions, we use the terms line and polyline to denote a one-dimensional polyhedron and the terms polygon and region to denote a two-dimensional polyhedron. We further assume that for each $k(0 \leq k \leq d)$, the set of k-dimensional polyhedra forms a data type, which leads us to the common collection of spatial data types \{Point, Line, Region, ... \}. Combined types sometimes also occur. An object o in a spatial database is usually defined by several nonspatial attributes and one attribute of some spatial data type. This spatial attribute describes the object's spatial extent o.G. In the spatial database literature, the terms geometry, shape, and spatial extension are often used instead of spatial extent. For the description of o.G one finds the terms shape descriptor, shape description, shape information, and geometric description, among others.

Indices often perform more efficiently when handling simple entries of the same size. One therefore often abstracts from the actual shape of a spatial object before inserting it into an index. This can be achieved by approximating the original data object with a simpler shape, such as a bounding box or a sphere. Given a minimum bounding interval $\mathrm{I}_{\mathrm{i}}(\mathrm{o})=\left[\mathrm{I}_{\mathrm{i}}, \mathrm{u}_{\mathrm{i}}\right]\left(\mathrm{I}_{\mathrm{i}}, \mathrm{u}_{\mathrm{i}} \in \mathrm{E}^{1}\right) \mathrm{de}-$ scribing the extent of the spatial object $o$ along dimension $i$, the d-dimensional minimum bounding box (MBB) is defined by $\mathrm{I}^{\mathrm{d}}(0)=\mathrm{I}_{1}(0) \times \mathrm{I}_{2}(0) \times \ldots \times$ $I_{d}(0)$.

An index may administer only the MBB of each object, together with a 
pointer to the object's database entry (object ID or object reference). With this design, the index produces only a set of candidate solutions (Figure 2). For each candidate obtained during this filter step, we have to decide whether the MBB is sufficient to guarantee that the object itself satisfies the search predicate. In those cases, the object can be added directly to the query result (dashed line). However, there are often cases where the MBB does not prove sufficient. In a refinement step we then must retrieve the exact shape information from secondary memory and test it against the predicate. If the predicate evaluates to true, the object is added to the query result; otherwise we have a false drop.

Another way of obtaining simple index entries is to represent the shape of each data object as the geometric union of simpler shapes (e.g., convex polygons with a bounded number of vertices). This approach is called decomposition.

We have mentioned the term efficiency several times so far without giving a formal definition. In the case of space efficiency, this can easily be done: the goal is to minimize the number of bytes occupied by the index. For time efficiency the situation is not so clear. Elapsed time is obviously what the user cares about, but one should keep in mind that the corresponding measurements greatly depend on implementation details, hardware utilization, and other external factors. In the literature, one therefore often finds a seemingly more objective performance measure: the number of disk accesses performed during a search. This approach, which has become popular with the B-tree, is based on the assumption that most searches are I/O-bound rather than CPU-bound-an assumption that is not always true in spatial data management, however. In applications where objects have complex shapes, the refinement step can incur major CPU costs and change the balance with I/O [Gaede 1995b; Hoel and Samet 1995]. Of course, one should keep the minimiza-

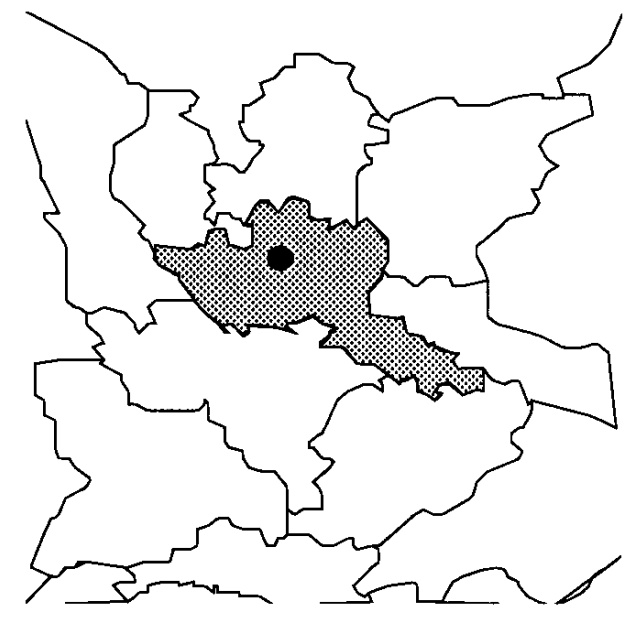

Figure 3. Point query.

tion of the number of disk accesses in mind as one design goal. Practical evaluations, however, should always give some information on elapsed times and the conditions under which they were achieved.

As noted previously, in contrast to relational databases, there exists neither a standard spatial algebra nor a standard spatial query language. The set of operators strongly depends on the given application domain, although some operators (such as intersection) are generally more common than others. Queries are often expressed by some extension of SQL that allows abstract data types to represent spatial objects and their associated operators [Roussopoulos and Leifker 1984; Egenhofer 1994]. The result of a query is usually a set of spatial data objects. In the remainder of this section, we give a formal definition of several of the more common spatial database operators. Figures 3 through 8 give some concrete examples.

Query 1 (Exact Match Query EMQ, Object Query). Given an object o' with spatial extent $\mathrm{O}^{\prime} . \mathrm{G} \subseteq \mathrm{E}^{\mathrm{d}}$, find all objects o with the same spatial extent as o':

$$
E M Q\left(O^{\prime}\right)=\left\{0 \mid O^{\prime} \cdot G=0 . G\right\} .
$$

Query 2 (Point Query PQ). Given a point $p \in E^{d}$, find all objects o overlap- 


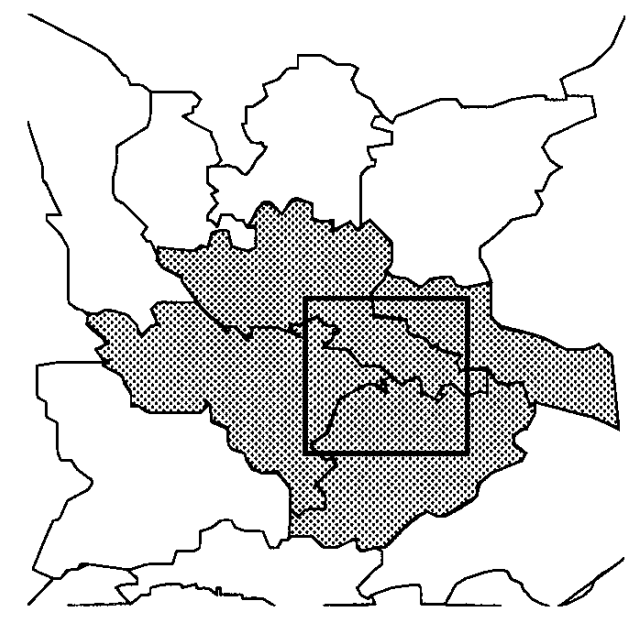

Figure 4. Window query.

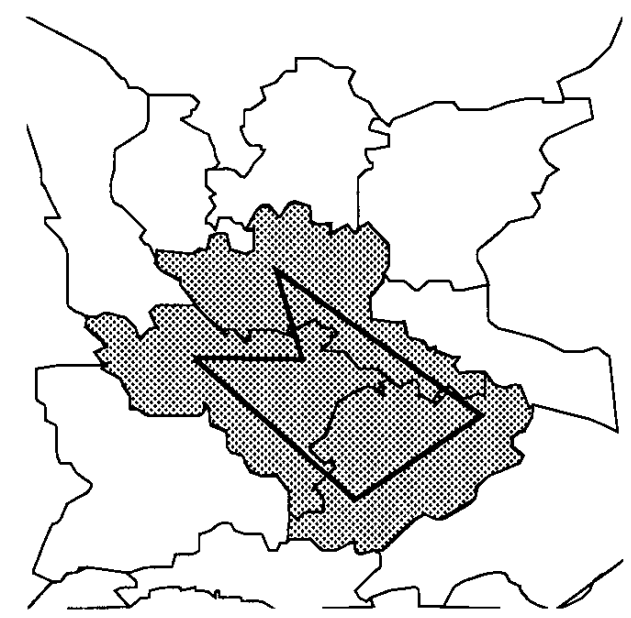

Figure 5. Intersection query.

ping $\mathrm{p}$ :

$$
P Q(p)=\{o \mid p \cap o . G=p\} .
$$

The point query can be regarded as a special case of several of the following queries, such as the intersection query, the window query, or the enclosure query.

Query 3 (Window Query WQ, Range Query). Given a d-dimensional interval $I^{d}=\left[I_{1}, u_{1}\right] \times\left[I_{2}, u_{2}\right] \times \ldots \times\left[I_{d}, u_{d}\right]$, find all objects 0 having at least one point in common with $\mathrm{I}^{\mathrm{d}}$ :

$$
W Q\left(I^{d}\right)=\left\{0 \mid I^{d} \cap 0 . G \neq \emptyset\right\} .
$$

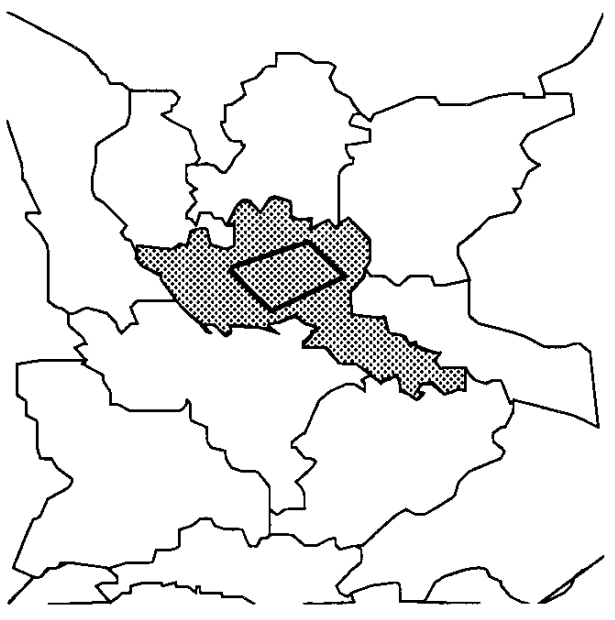

Figure 6. Enclosure query.

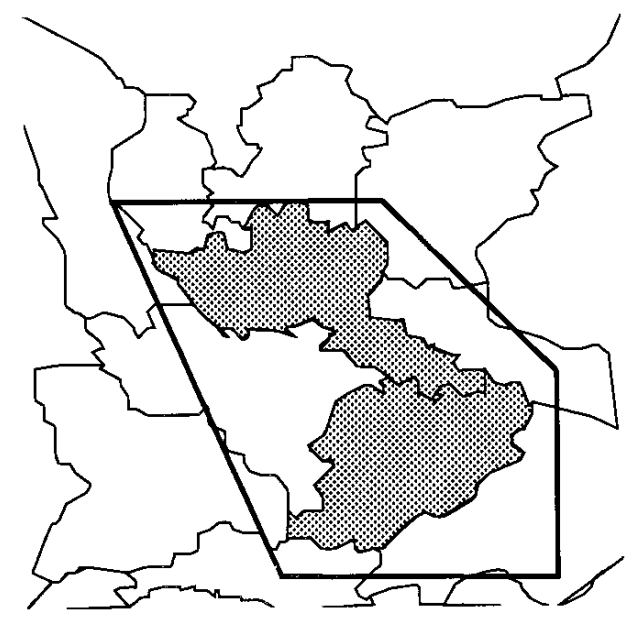

Figure 7. Containment query.

The query implies that the window is iso-oriented; that is, its faces are parallel to the coordinate axes. A more general variant is the region query that permits search regions to have arbitrary orientations and shapes.

Query 4 (Intersection Query IQ, Region Query, Overlap Query). Given an object $o^{\prime}$ with spatial extent $\mathrm{O}^{\prime} . \mathrm{G} \subseteq \mathrm{E}^{\mathrm{d}}$, find all objects $O$ having at least one point in common with $\mathrm{o}^{\prime}$ :

$$
\mathrm{IQ}\left(\mathrm{o}^{\prime}\right)=\left\{\mathrm{o} \mid \mathrm{O}^{\prime} . \mathrm{G} \cap \mathrm{o.G} \neq \emptyset\right\} .
$$

Query 5 (Encl osure Query EQ). Given an object $\mathrm{O}^{\prime}$ with spatial extent $\mathrm{O}^{\prime} . \mathrm{G} \subseteq$ 


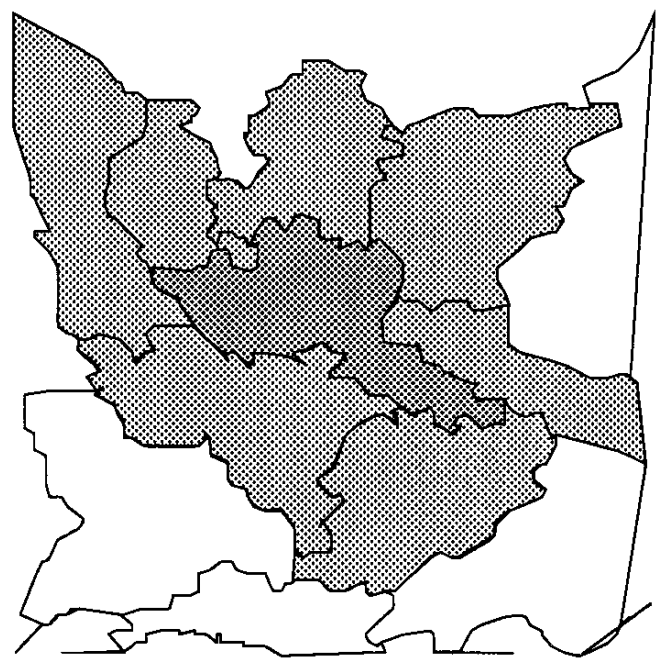

Figure 8. Adjacency query.

$E^{d}$, find all objects o enclosing $\mathrm{O}^{\prime}$ :

$$
E Q\left(o^{\prime}\right)=\left\{0 \mid\left(o^{\prime} . G \cap O . G\right)=o^{\prime} . G\right\} \text {. }
$$

Query 6 (Containment Query CQ). Given an object $\mathrm{O}^{\prime}$ with spatial extent $\mathrm{O}^{\prime} . \mathrm{G} \subseteq \mathrm{E}^{\mathrm{d}}$, find all objects o enclosed by $\mathrm{O}^{\prime}$ :

$$
\mathrm{CQ}\left(\mathrm{o}^{\prime}\right)=\left\{\mathrm{O} \mid\left(\mathrm{o}^{\prime} . \mathrm{G} \cap \mathrm{O} . \mathrm{G}\right)=\mathrm{o.G}\right\} .
$$

The enclosure and the containment query are duals of each other. They are both more restrictive formulations of the intersection query by specifying the result of the intersection to be one of the two inputs.

Query 7 (Adjacency Query AQ). Given an object $o^{\prime}$ with spatial extent $\mathrm{o}^{\prime} \cdot G \subseteq$ $E^{d}$, find all objects $\mathrm{o}$ adjacent to $\mathrm{o}^{\prime}$ :

$$
\begin{aligned}
A Q\left(o^{\prime}\right)=\{0 \mid 0 . G & \cap o^{\prime} . G \neq \emptyset \\
& \left.\wedge 0^{\prime} . G^{\circ} \cap 0 . G^{\circ}=\emptyset\right\} .
\end{aligned}
$$

Here $\mathrm{O}^{\prime} . \mathrm{G}^{\circ}$ and $\mathrm{O} . \mathrm{G}^{\circ}$ denote the interiors of the spatial extents $0^{\prime} . G$ and o.G, respectively.

Query 8 (Nearest-Neighbor Query NNQ). Given an object o' with spatial extent $\mathrm{O}^{\prime} . \mathrm{G} \subseteq \mathrm{E}^{\mathrm{d}}$, find all objects $\mathrm{O}$ having a minimum distance from o':

$$
\begin{aligned}
N N Q\left(o^{\prime}\right)=\left\{0 \mid \forall 0^{\prime \prime}\right. & : \operatorname{dist}\left(o^{\prime} . G, o . G\right) \\
& \left.\leq \operatorname{dist}\left(o^{\prime} . G, o^{\prime \prime} . G\right)\right\} .
\end{aligned}
$$

The distance between extended spatial data objects is usually defined as the distance between their closest points. Common distance functions for points include the Euclidean and the Manhattan distance.

Besides spatial selections, as exemplified by Queries 1 through 8, the spatial join is one of the most important spatial operations and can be defined as follows [Günther 1993]:

Query 9 (Spatial J oin). Given two collections $\mathrm{R}$ and $\mathrm{S}$ of spatial objects and a spatial predicate $\theta$, find all pairs of objects $\left(0, o^{\prime}\right) \in R \times S$ where $\theta\left(0 . G, o^{\prime} . G\right)$ evaluates to true:

$$
\begin{aligned}
\mathrm{R} \bowtie_{\theta} \mathrm{S}=\left\{\left(\mathrm{o}, \mathrm{o}^{\prime}\right) \mid \mathrm{o}\right. & \in \mathrm{R} \wedge \mathrm{o}^{\prime} \\
& \left.\in \mathrm{S} \wedge \theta\left(\mathrm{o} . \mathrm{G}, \mathrm{o}^{\prime} . \mathrm{G}\right)\right\} .
\end{aligned}
$$

As for the spatial predicate $\theta$, a brief survey of the literature ${ }^{1}$ yields a wide variety of possibilities, including

intersects $(\cdot)$

contains $(\cdot)$

is_enclosed_by (.)

distance $(\cdot) \mathrm{q}$, with $\Theta \in\{=, \leq,<, \geq,>\}$ and $\mathrm{q} \in \mathrm{E}^{1}$

northwest $(\cdot)$

$\operatorname{adjacent}(\cdot)$.

A closer inspection of these spatial predicates shows that the intersection join $R \triangleright{ }_{\text {intersects }} S$ plays a crucial role for the computation in virtually all these cases [Gaede and Riekert 1994]. For predicates such as contains, encloses, or adjacent, for example, the intersection join is an efficient filter that yields a set of candidate solutions typically much

\footnotetext{
${ }^{1}$ See Orenstein [1986], Becker [1992], Rotem [1991], Günther [1993], Brinkhoff et al. [1993a], Gaede and Riekert [1994], Brinkhoff [1994], Lo and Ravishankar [1994], Aref and Samet [1994], Papadias et al. [1995] and Günther et al. [1998].
} 
smaller than the Cartesian product $\mathrm{R} \times \mathrm{S}$.

\section{BASIC DATA STRUCTURES}

\subsection{One-Dimensional Access Methods}

Classical one-dimensional access methods are an important foundation for almost all multidimensional access methods. Although the related surveys by Knott [1975] and Comer [1979] are somewhat dated, they represent good coverage of the different approaches. In practice, the most common one-dimensional structures include linear hashing [Litwin 1980; Larson 1980], extendible hashing [Fagin et al. 1979], and the B-tree [Bayer and McCreight 1972]. $\mathrm{Hi}$ erarchical access methods such as the B-tree are scalable and behave well in the case of skewed input; they are nearly independent of the distribution of the input data. This is not necessarily true for hashing techniques, whose performance may degenerate depending on the given input data and hash function. This problem is aggravated by the use of order-preserving hash functions [Orenstein 1983; Garg and Gotlieb 1986] that try to preserve neighborhood relationships between data items in order to support range queries. As a result, highly skewed data keep accumulating at a few selected locations in image space.

3.1.1 Linear Hashing [Larson 1980; Litwin 1980]. Linear hashing divides the universe [A, B ) of possible hash values into binary intervals of size $(B-$ A) $/ 2^{k}$ or $(B-A) / 2^{k+1}$ for some $k \geq 0$. Each interval corresponds to a bucket, that is, a collection of records stored on a disk page. $t \in[A, B)$ is a pointer that separates the smaller intervals from the larger ones: all intervals of size $(B-$ A) $/ 2^{\mathrm{k}}$ are to the left of $t$ and all intervals of size $(B-A) / 2^{k+1}$ are to the right of $t$. If a bucket reaches its capacity due to an insertion, the interval $[t, t+(B-$ A) $/ 2^{\mathrm{k}}$ ) is split into two subintervals of equal size, and $t$ is advanced to the next large interval remaining. Note that the split interval need not be the same interval as the one that caused the split; consequently, there is no guarantee that the split relieves the bucket in question from its overload. If an interval contains more objects than bucket capacity permits, the overload is stored on an overflow page, which is linked to the original page. When $t=B$, the file has doubled and all intervals have the same length $(B-A) / 2^{k+1}$. In this case we reset the pointer $t$ to $A$ and resume the split procedure for the smaller intervals.

3.1.2 Extendible Hashing [Fagin et al. 1979]. As does linear hashing, extendible hashing organizes the data in binary intervals, here called cells. Overflow pages are avoided in extendible hashing by using a central directory. Each cell has an index entry in that directory; it initially corresponds to one bucket. If during an insertion a bucket at maximal depth exceeds its maximum capacity, all cells are split into two. New index entries are created and the directory doubles in size. Since each bucket was not at full capacity before the split, it may now be possible to fit more than one cell in the same bucket. In that case, adjacent cells are regrouped in data regions and stored on the same disk page. In the case of skewed data this may lead to a situation where numerous directory entries exist for the same data region (and therefore the same disk page). Even in the case of uniformly distributed data, the average directory size is $\Theta\left(n^{1+1 / b}\right)$ and therefore superlinear [Flajolet 1983]. Here b denotes the bucket size and $\mathrm{n}$ is the number of index entries. Exact match searches take no more than two page accesses: one for the directory and one for the bucket with the data. This is more than the best-case performance of linear hashing, but better than the worst case.

Besides the potentially poor space utilization of the index, extendible hashing also suffers from a nonincremental 
growth of the index due to the doubling steps. To address these problems, Lomet [1983] proposed a technique called bounded-index extendible hashing. In this proposal, the index grows as in extendible hashing until its size reaches a predetermined maximum; that is, the index size is bounded. Once this limit is reached while inserting new items, bounded-index extendible hashing starts doubling the data bucket size rather than the index size.

3.1.3 The B-Tree [Bayer and McCreight 1972]. Other than hashing schemes, the B-tree and its variants [Comer 1979] organize the data in a hierarchical manner. B-trees are balanced trees that correspond to a nesting of intervals. Each node $\nu$ corresponds to a disk page $\mathrm{D}(\nu)$ and an interval I $(\nu)$. If $\nu$ is an interior node then the intervals $\mathrm{I}\left(\nu_{\mathrm{i}}\right)$ corresponding to the immediate descendants of $\nu$ are mutually disjoint subsets of $\mathrm{I}(\nu)$. Leaf nodes contain pointers to data items; depending on the type of B-tree, interior nodes may do so as well. B-trees have an upper and lower bound for the number of descendants of a node. The lower bound prevents the degeneration of trees and leads to an efficient storage utilization. Nodes whose number of descendants drops bel ow the lower bound are del eted and their contents distributed among the adjacent nodes at the same tree level. The upper bound follows from the fact that each tree node corresponds to exactly one disk page. If during an insertion a node reaches its capacity, it is split in two. Splits may propagate up the tree. As the size of the intervals depends on the given data (and the insertion sequence), the B-tree is an adaptive data structure. For uniformly distributed data, however, extendible as well as linear hashing outperform the B-tree on the average for exact match queries, insertions, and deletions.

\subsection{Main Memory Structures}

Early multidimensional access methods did not take into account paged second- ary memory and are therefore less suited for large spatial databases. In this section, we review several of these fundamental data structures, which are adapted and incorporated in numerous multidimensional access methods. To illustrate the methods, we introduce a small scenario that we use as a running example throughout this survey. The scenario, depicted in Figure 9, contains 10 points pi and 10 polygons ri, randomly distributed in a finite two-dimensional universe. To represent polygons, we often use their centroids ci (not pictured) or their minimum bounding boxes (MBBs) mi. Note that the quality of the MBB approximation varies considerably. The MBB m8, for example, provides a fairly tight fit, whereas $r 5$ is only about half as large as its MBB m5.

3.2.1 The k-d-Tree [Bentley 1975, 1979]. One of the most prominent $d$ dimensional data structures is the k-dtree. The k-d-tree is a binary search tree that represents a recursive subdivision of the universe into subspaces by means of (d - 1)-dimensional hyperplanes. The hyperplanes are iso-oriented, and their direction alternates among the $d$ possibilities. For $d=3$, for example, splitting hyperplanes are alternately perpendicular to the $x-, y-$, and $z$-axes. Each splitting hyperplane has to contain at least one data point, which is used for its representation in the tree. Interior nodes have one or two descendants each and function as discriminators to guide the search. Searching and insertion of new points are straightforward operations. Deletion is somewhat more complicated and may cause a reorganization of the subtree bel ow the data point to be deleted.

Figure 10 shows a k-d-tree for the running example. Because the tree can only handle points, we represent the polygons by their centroids ci. The first splitting line is the vertical line crossing c3. We therefore store c3 in the root of the corresponding k-d-tree. The next splits occur along horizontal lines cross- 


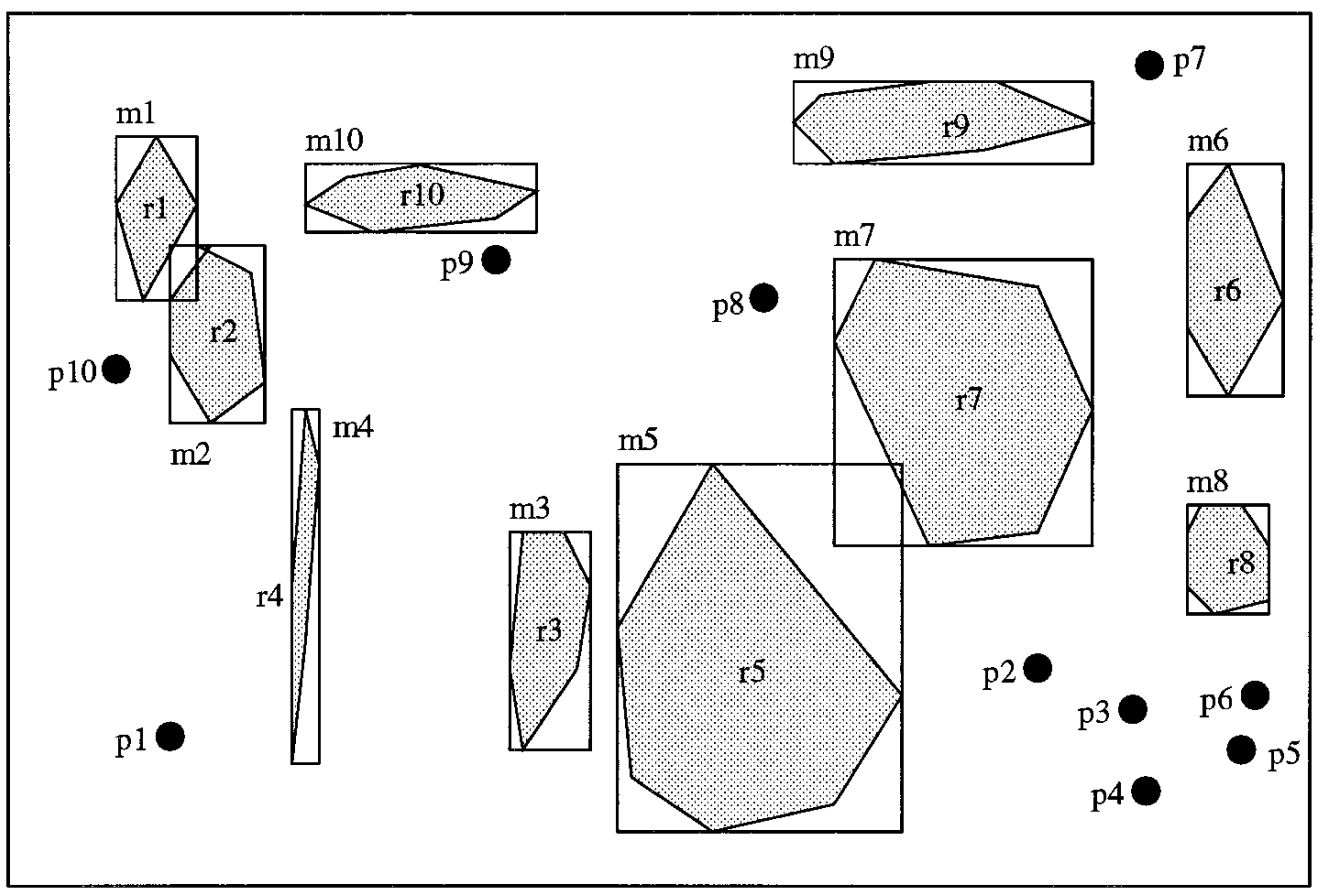

Figure 9. Running example.
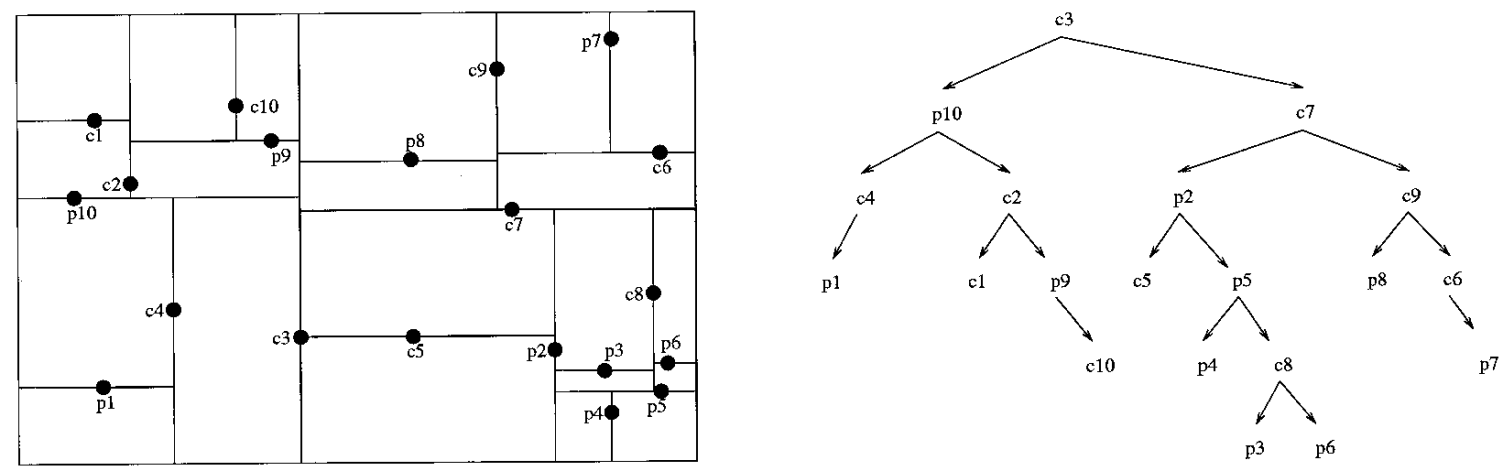

Figure 10. k-d-tree.

ing p10 (for the left subtree) and c7 (for the right subtree), and so on.

One disadvantage of the $\mathrm{k}$-d-tree is that the structure is sensitive to the order in which the points are inserted. Another one is that data points are scattered all over the tree. The adaptive k-d-tree [Bentley and Friedman 1979] mitigates these problems by choosing a split such that one finds about the same number of elements on both sides. Although the splitting hyperplanes are still parallel to the axes, they need not contain a data point and their directions need not be strictly alternating anymore. As a result, the split points are not part of the input data; all data points are stored in the leaves. Interior nodes contain the dimension (e.g., $\mathrm{x}$ or y) and the coordinate of the corresponding split. Splitting is continued recursively until each subspace contains only a certain number of points. The adaptive k-d-tree is a rather static structure; it is obviously difficult to keep the tree balanced in the presence of frequent insertions and deletions. The structure works best if all the data are known a 

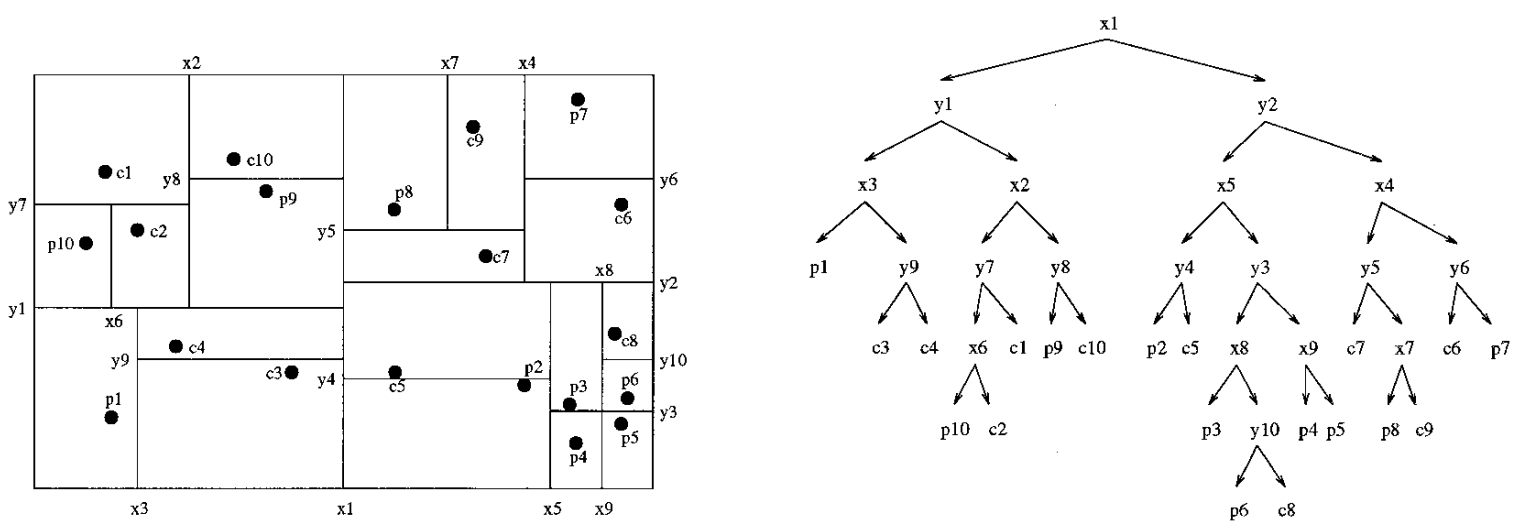

Figure 11. Adaptive k-d-tree.

priori and if updates are rare. Figure 11 shows an adaptive k-d-tree for the running example. Note that the tree still depends on the order of insertion.

Another variant of the k-d-tree is the bintree [Tamminen 1984]. This structure partitions the universe recursively into d-dimensional boxes of equal size until each one contains only a certain number of points. Even though this kind of partitioning is less adaptive, it has several advantages, such as the implicit knowledge of the partitioning hyperplanes. In the remainder of this article, we encounter several other structures based on this kind of partitioning.

A disadvantage common to all $\mathrm{k}$-dtrees is that for certain distributions no hyperplane can be found that splits the data points evenly [Lomet and Salzberg 1989]. By introducing a more flexible partitioning scheme, the following BSPtree avoids this problem completely.

3.2.2 The BSP-Tree [Fuchs et al. 1980, 1983]. Splitting the universe only along iso-oriented hyperplanes is a severe restriction in the schemes presented so far. Allowing arbitrary orientations gives more flexibility to find a hyperplane that is well suited for the split. A well-known example for such a method is the binary space partitioning (BSP)-tree. Like k-d-trees, BSP-trees are binary trees that represent a recursive subdivision of the universe into subspaces by means of $(d-1)$-dimensional hyperplanes. Each subspace is subdivided independently of its history and of the other subspaces. The choice of the partitioning hyperplanes depends on the distribution of the data objects in a given subspace. The decomposition usually continues until the number of objects in each subspace is below a given threshold.

The resulting partition of the universe can be represented by a BSP-tree in which each hyperplane corresponds to an interior node of the tree and each subspace corresponds to a leaf. Each leaf stores references to those objects that are contained in the corresponding subspace. Figure 12 shows a BSP-tree for the running example with no more than two objects per subspace.

In order to perform a point query, we insert the search point into the root of the tree and determine on which side of the corresponding hyperplane it is located. N ext, we insert the point into the corresponding subtree and proceed recursively until we reach a leaf of the tree. Finally, we examine the data objects in the corresponding subspace to see whether they contain the search point. The range search algorithm is a straightforward generalization.

BSP-trees can adapt well to different data distributions. However, they are typically not balanced and may have very deep subtrees, which has a nega- 


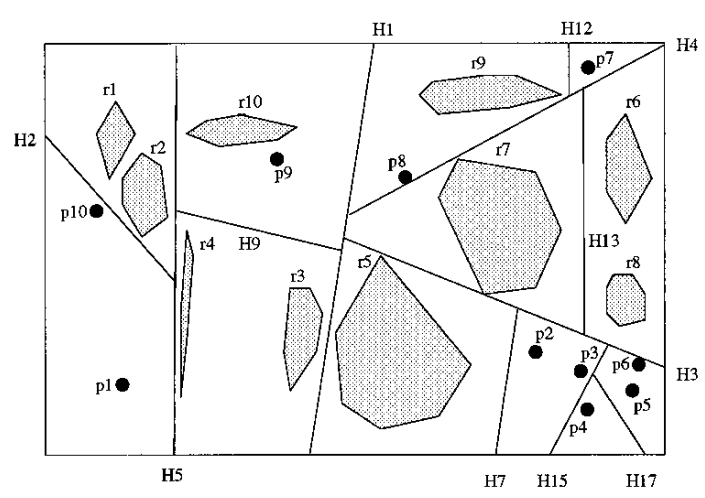

Figure 12. BSP-tree.

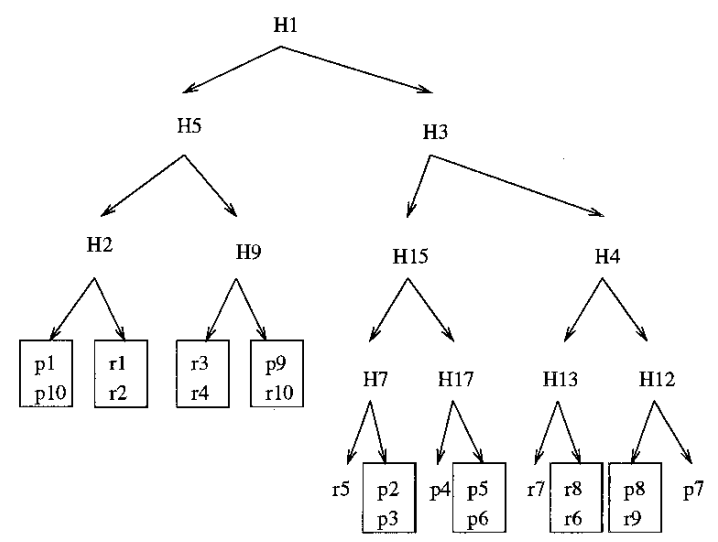

tive impact on the tree performance. BSP-trees also have higher space requirements, since storing an arbitrary hyperplane per split occupies more storage space than a simple discriminator, which is typically just a real number.

3.2.3 The BD-Tree [Ohsawa and Sakauchi 1983]. The BD-tree is a binary tree representing a subdivision of the data space into interval-shaped regions. Each of those regions is encoded in a bit string and associated with one of the BD-tree nodes. Here, these bit strings are called DZ-expressions; they are also known as Peano codes, ST MortonNumber, or z-values (cf. Section 5.1.2).

Given a region $\mathrm{R}$, one computes the corresponding DZ-expression as follows. For simplicity we restrict this presentation to the two-dimensional case; we also assume that the first subdividing hyperplane is a vertical line. If $\mathrm{R}$ lies to the left of that line, the first bit of the corresponding DZ-expression is 0; otherwise it is 1 . In the next step, we subdivide the subspace containing $R$ by $a$ horizontal line. If $\mathrm{R}$ lies bel ow that line, the second bit of the DZ-expression is 0 , otherwise it is 1 . As this decomposition progresses, we obtain one bit per splitting line. Bits at odd positions refer to vertical lines and bits at even positions to horizontal lines, which explains why this scheme is often referred to as bit interleaving.
To avoid the storage utilization problems that are often associated with a strictly regular partitioning, the BDtree employs a more flexible splitting policy. Here one can split a node by making an interval-shaped excision from the corresponding region. The two child nodes of the node to be split will then have different interpretations: one represents the excision; the other one represents the remainder of the original region. Note that the remaining region is no longer interval-shaped. With this policy, the BD-tree can guarantee that, after node splitting, each of the data buckets contains at least one third of the original entries.

Figure 13 shows a BD-tree for the running example. An excision is always represented by the left child of the node that was split.

For an exact match we first compute the full bit-interleaved prefix of the search record. Starting from the root, we recursively compare this prefix with the stored DZ-expressions of each internal node. If it matches, we follow the corresponding link; otherwise we follow the other link until we reach the leaf level of the BD-tree. More sophisticated algorithms were proposed later by Dandamudi and Sorenson [1986, 1991].

3.2.4 The Quadtree. The quadtree with its many variants is closely related to the k-d-tree. For an extensive discussion of this structure, see Samet [1984, 

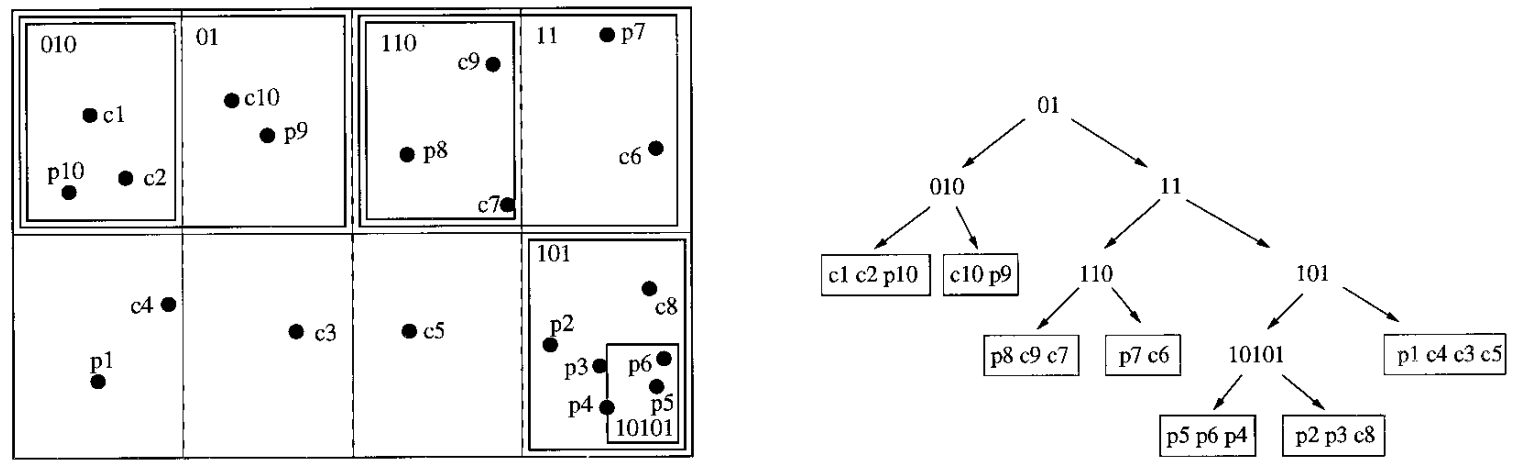

Figure 13. BD-tree.

1990a,b]. Although the term quadtree usually refers to the two-dimensional variant, the basic idea applies to an arbitrary $d$. Like the k-d-tree, the quadtree decomposes the universe by means of iso-oriented hyperplanes. An important difference, however, is the fact that quadtrees are not binary trees anymore. In d dimensions, the interior nodes of a quadtree have $2^{\mathrm{d}}$ descendants, each corresponding to an interval-shaped partition of the given subspace. These partitions do not have to be of equal size, although that is often the case. For $d=2$, for example, each interior node has four descendants, each corresponding to a rectangle. These rectangles are typically referred to as the NW, NE, SW, and SE (northwest, etc.) quadrants. The decomposition into subspaces is usually continued until the number of objects in each partition is bel ow a given threshold. Quadtrees are therefore not necessarily balanced; subtrees corresponding to densely populated regions may be deeper than others.

Searching in a quadtree is similar to searching in an ordinary binary search tree. At each level, one has to decide which of the four subtrees need be included in the future search. In the case of a point query, typically only one subtree qualifies, whereas for range queries there are often several. We repeat this search step recursively until we reach the leaves of the tree.

Finkel and Bentley [1974] proposed one of the first quadtree variants: the point quadtree, essentially a multidimensional binary search tree. The point quadtree is constructed consecutively by inserting the data points one by one. For each point, we first perform a point search. If we do not find the point in the tree, we insert it into the leaf node where the search has terminated. The corresponding partition is divided into $2^{d}$ subspaces with the new point at the center. The deletion of a point requires the restructuring of the subtree below the corresponding quadtree node. A simple way to achieve this is to reinsert all points into the subtree. Figure 14 shows a two-dimensional point quadtree for the running example.

Another popular variant is the region quadtree [Samet 1984]. Region quadtrees are based on a regular decomposition of the universe; that is, the $2^{\mathrm{d}}$ subspaces resulting from a partition are always of equal size. This greatly facilitates searches. For the running example, Figure 15 shows how region quadtrees can be used to represent sets of points. Here the threshold for the number of points in any given subspace was set to one. In more complex versions of the region quadtree, such as the PM quadtree [Samet and Webber 1985], it is also possible to store polygonal data directly. PM quadtrees divide the quadtree regions (and the data objects in them) until they contain only a small number of polygon edges or vertices. These edges or vertices (which together form 

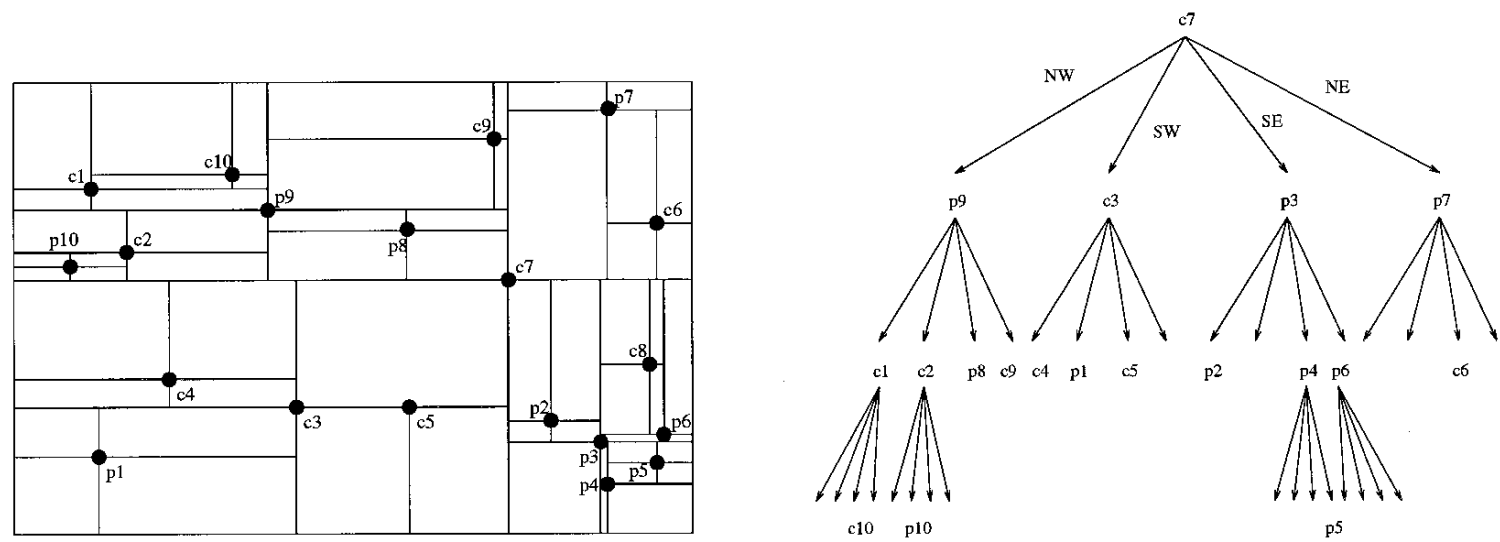

Figure 14. Point quadtree.
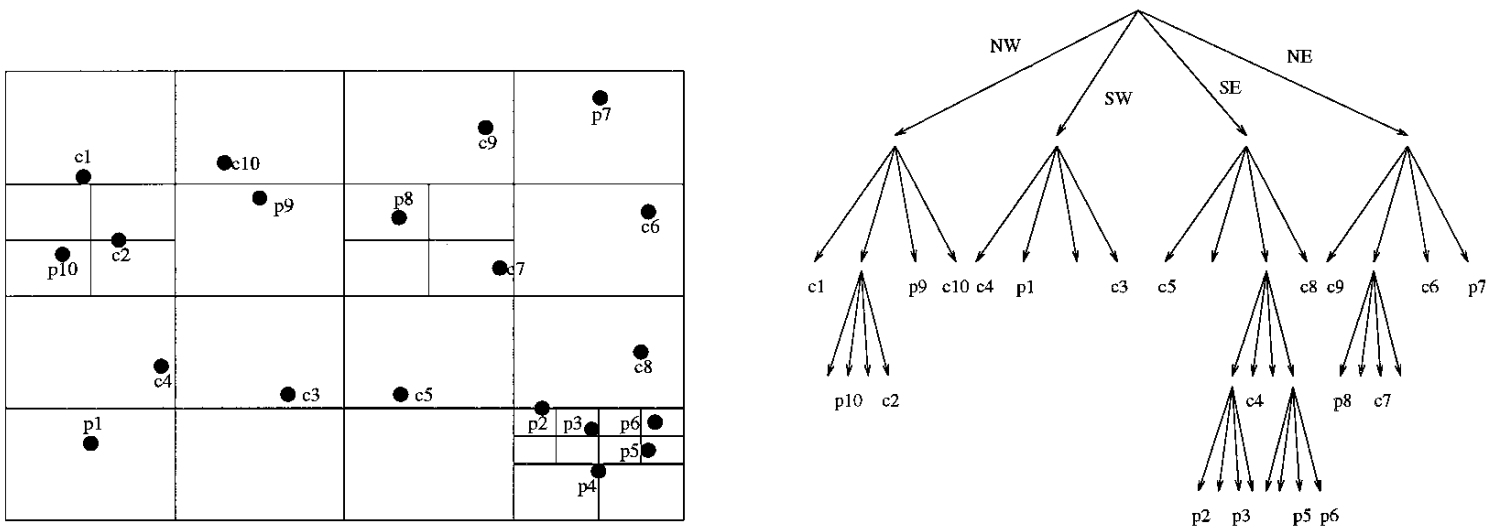

Figure 15. Region quadtree.

an exact description of the data objects) are then attached to the leaves of the tree. Another class of quadtree structures has been designed for the management of collections of rectangles; see Samet [1988] for a survey.

\section{POINT ACCESS METHODS}

The multidimensional data structures presented in the previous section do not take secondary storage management into account explicitly. They were originally designed for main memory applications where all the data are available without accessing the disk. Despite growing main memories, this is of course not always the case. In many spatial database applications, such as geography, the amount of data to be managed is notoriously large. One can certainly use main memory structures for data that reside on disk, but their performance is often considerably below the optimum because there is no control over how the operating system performs the disk accesses. The access methods presented in this and the following section have been designed with secondary storage management in mind. Their operations are closely coordinated with the operating system to ensure that overall performance is optimized.

As mentioned before, we first present a selection of point access methods. Usually, the points in the database are organized in a number of buckets, each of which corresponds to a disk page and to some subspace of the universe. The subspaces (often called data regions, bucket regions, or simply regions, even 
Table 1. Classification of PAMs Following Seeger and Kriegel [1990]

\begin{tabular}{|c|c|c|c|}
\hline \multicolumn{3}{|c|}{ property } & \multirow{2}{*}{ point access method } \\
\hline intervals & complete & disjoint & \\
\hline$x$ & $x$ & $x$ & $\begin{array}{l}\text { quadtree [Finkel and Bentley 1974; Samet 1984], k-d-B tree } \\
\text { [Robinson 1981], EXCELL [Tamminen 1982], interpolation } \\
\text { hashing [Burkhard 1983], multidimensional extendible hashing } \\
\text { [Otoo 1984], grid file [Nievergelt et al. 1984], balanced multi- } \\
\text { dimensional two-level grid file [Hinrichs 1985], interpolation- } \\
\text { based grid file [Ouksel 1985], extendible hash tree [Otoo 1986], } \\
\text { MOLHPE [Kriegel and Seeger 1986], PLOP-hashing [Kriegel } \\
\text { and Seeger 1988], quantile hashing [Kriegel and Seeger 1989], } \\
\text { LSD-tree [Henrich et al. 1989] }\end{array}$ \\
\hline $\bar{x}$ & $\bar{x}$ & & twin grid file [Hutflesz et al. 1988] \\
\hline $\bar{x}$ & & $\bar{x}$ & $\begin{array}{l}\text { multilevel grid file [Whang and Krishnamurthy 1985], buddy } \\
\text { tree [Seeger and Kriegel 1990] }\end{array}$ \\
\hline & $x$ & $x$ & $\begin{array}{l}\text { BSP-tree [Fuchs et al. 1980], BD-tree [Ohsawa and Sakauchi } \\
\text { 1983], BANG file [Freeston 1987], hB-tree [Lomet and } \\
\text { Salzberg 1989] }\end{array}$ \\
\hline
\end{tabular}

though their dimension may be greater than two) need not be rectilinear, although they often are. The buckets are accessed by means of a search tree or some d-dimensional hash function.

The grid file [Nievergelt et al. 1984], for example, uses a directory and a gridlike partition of the universe to answer an exact match query with exactly two disk accesses. Furthermore, there are multidimensional hashing schemes [Tamminen 1982; Kriegel and Seeger 1986, 1988], multilevel grid files [Whang and Krishnamurthy 1985; Hutflesz et al. 1988b], and hash trees [Ouksel 1985; Otoo 1985], which organize the directory as a tree structure. Tree-based access methods are usually a generalization of the B-tree to higher dimensions, such as the k-d-B-tree [Robinson 1981] or the hB-tree [Lomet and Salzberg 1989].

In the remainder of this section, we first discuss the approaches based on hashing, followed by hierarchical (treebased) methods, and space-filling curves. This classification is hardly unambiguous, especially in the presence of an increasing number of hybrid approaches that attempt to combine the advantages of several different techniques. Our approach resembles the classification of Samet [1990], who dis- tinguishes between hierarchical methods (point/region quadtrees, k-d-trees, range trees) and bucket methods (grid file, EXCELL). His discussion of the former is primarily in the context of main memory applications. Our presentation focuses throughout on structures that take secondary storage management into account.

Another interesting taxonomy has been proposed by Seeger and Kriegel [1990], who classify point access methods by the properties of the bucket regions (Table 1). First, they may be pairwise disjoint or they may have mutual overlaps. Second, they may have the shape of an interval (box) or be of some arbitrary polyhedral shape. Third, they may cover the complete universe or just those parts that contain some data objects. This taxonomy results in eight classes, four of which are populated by existing access methods.

\subsection{Multidimensional Hashing}

Although there is no total order for objects in two and higher-dimensional space that completely preserves spatial proximity, there have been numerous attempts to construct hashing functions that preserve proximity at least to some extent. The goal of all these heuristics is 


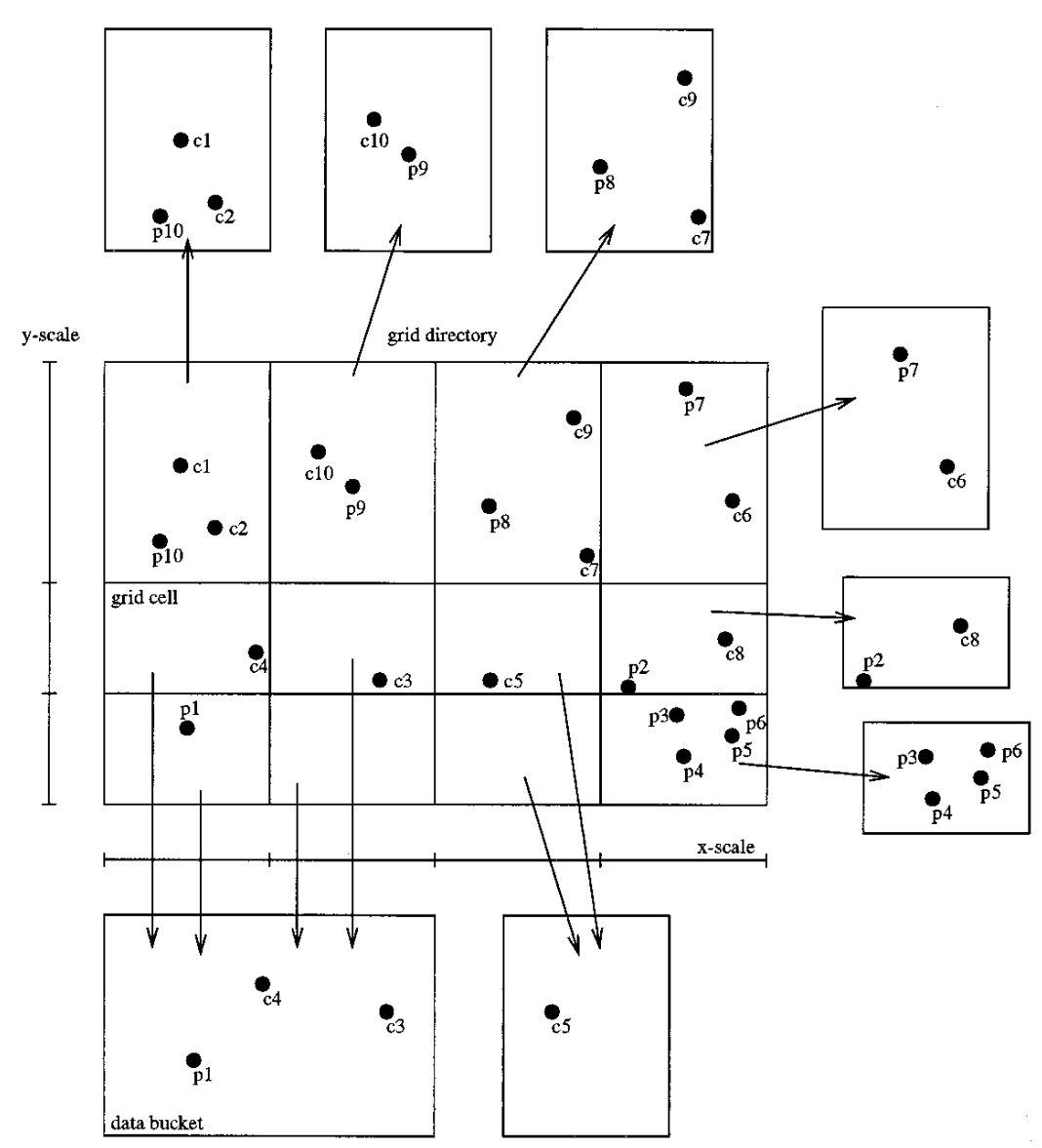

Figure 16. Grid file.

that objects located close to each other in original space should be likely to be stored close together on the disk. This could contribute substantially to minimizing the number of disk accesses per range query. We begin our presentation with several structures based on extendible hashing. Structures based on linear hashing are discussed in Section 4.1.5. The discussion of two hybrid methods, the BANG file and the buddy tree, is postponed until Section 4.2.

4.1.1 The Grid File (Nievergelt et al. 1981]. As a typical representative of an access method based on hashing, we first discuss the grid file and some of its variants. $^{2}$ The grid file superimposes a

2 See Hinrichs [1985], Ouksel [1985], Whang and Krishnamurthy [1985], Six and Widmayer [1988], and Blanken et al. [1990]. d-dimensional orthogonal grid on the universe. Because the grid is not necessarily regular, the resulting cells may be of different shapes and sizes. A grid directory associates one or more of these cells with data buckets, which are stored on one disk page each. Each cell is associated with one bucket, but a bucket may contain several adjacent cells. Since the directory may grow large, it is usually kept on secondary storage. To guarantee that data items are al ways found with no more than two disk accesses for exact match queries, the grid itself is kept in main memory, represented by d one-dimensional arrays called scales.

Figure 16 shows a grid file for the running example. We assume bucket capacity to be four data points. The center of the figure shows the directory with 
scales on the $x$ - and $y$-axes. The data points are displayed in the directory for demonstration purposes only; they are not, of course, stored there. In the lower left part, four cells are combined into a single bucket, represented by four pointers to a single page. There are thus four directory entries for the same page, which illustrates a well-known problem of the grid file: it suffers from a superlinear growth of the directory even for data that are uniformly distributed [Regnier 1985; Widmayer 1991]. The bucket region containing the point c5 could have been merged with one of the neighboring buckets for better storage utilization. We present various merging strategies later, when we discuss the deletion of data points.

To answer an exact match query, one first uses the scales to locate the cell containing the search point. If the appropriate grid cell is not in main memory, one disk access is necessary. The loaded cell contains a reference to the page where possibly matching data can be found. Retrieving this page may require another disk access. Altogether, no more than two page accesses are necessary to answer this query. For a range query, one must examine all cells that overlap the search region. After eliminating duplicates, one fetches the corresponding data pages into memory for more detailed inspection.

To insert a point, one first performs an exact match query to locate the cell and the data page $\nu_{\mathrm{i}}$ where the entry should be inserted. If there is sufficient space left on $\nu_{\mathrm{i}}$, the new entry is inserted. If not, we have to distinguish two cases, depending on the number of grid cells that point to the data page where the new data item is to be inserted. If there are several, one checks whether an existing hyperplane stored in the scales can be used for splitting the data page successfully. If so, a new data page is allocated and the data points are distributed accordingly among the data pages. If none of the existing hyperplanes is suitable, or if only one grid cell points to the data page in question, a splitting hyperplane $\mathrm{H}$ is introduced and a new data page $\nu_{\mathrm{j}}$ is allocated. The new entry and the entries of the original page $\nu_{\mathrm{i}}$ are redistributed among $\nu_{\mathrm{i}}$ and $\nu_{\mathrm{i}}$, depending on their location relative to $\mathrm{H}$. $\mathrm{H}$ is inserted into the corresponding scale; all cells that intersect $\mathrm{H}$ are split accordingly. Splitting is therefore not a local operation and can lead to superlinear directory growth even for uniformly distributed data [Regnier 1985; Freeston 1987; Widmayer 1991].

Deletion is not a local operation either. With the deletion of an entry, the storage utilization of the corresponding data page may drop below the given threshold. Depending on the current partitioning of space, it may then be possible to merge this page with a neighbor page and to drop the partitioning hyperplane from the corresponding scale. Depending on the implementation of the grid directory, merging may require a complete directory scan [Hinrichs 1985]. Hinrichs discusses several methods for finding candidates with which a given data bucket can merge, including the neighbor system and the multidimensional buddy system. The neighbor system allows merging two adjacent regions if the result is a rectangular region again. In the buddy system, two adjacent regions can be merged provided that the joined region can be obtained by a regular binary subdivision of the universe. Both systems are not able to eliminate completely the possibility of a deadlock, in which case no merging is feasible because the resulting bucket region would not be box-shaped [Hinrichs 1985; Seeger and Kriegel 1990].

For a theoretical analysis of the grid file and some of its variants, see Regnier [1985] and Becker [1992]. Regnier shows in particular that the grid file's average directory size for uniformly distributed data is $\Theta\left(\mathrm{n}^{1+(\mathrm{d}-1) /(\mathrm{db}+1)}\right)$, where $\mathrm{b}$ is bucket size. He also proves that the average space occupancy of the data buckets is about 69\% (In 2). 


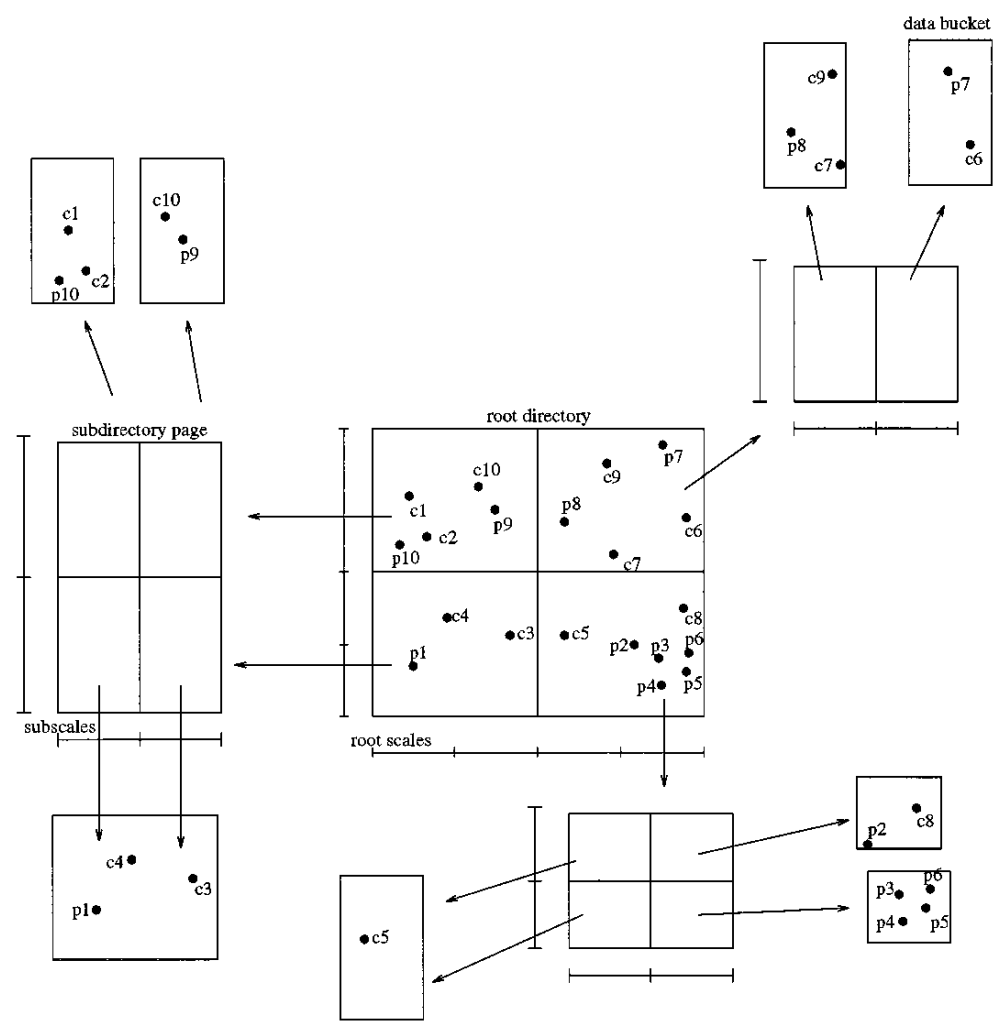

Figure 17. Two-level grid file.

4.1.2 EXCELL [Tamminen 1982]. Closely related to the grid file is the EXCELL method (Extendible CELL) proposed by Tamminen [1982]. In contrast to the grid file, where the partitioning hyperplanes may be spaced arbitrarily, the EXCELL method decomposes the universe regularly: all grid cells are of equal size. In order to maintain this property in the presence of insertions, each new split results in the halving of all cells and therefore in the doubling of the directory size. To alleviate this problem, Tamminen [1983] later suggested a hierarchical method, similar to the multilevel grid file of Whang and Krishnamurthy [1985]. Overflow pages are introduced to limit the depth of the hierarchy.

4.1.3 The Two-Level Grid File [Hinrichs 1985]. The basic idea of the twolevel grid file is to use a second grid file to manage the grid directory. The first of the two levels is called the root direc- tory, which is a coarsened version of the second level, the actual grid directory. Entries of the root directory contain pointers to the directory pages of the lower level, which in turn contain pointers to the data pages. By having a second level, splits are often confined to the subdirectory regions without affecting too much of their surroundings. Even though this modification leads to a slower directory growth, it does not solve the problem of superlinear directory size. Furthermore, Hinrichs implicitly assumes that the second level can be kept in main memory, so that the two-disk access principle still holds. Figure 17 shows a two-level grid file for the running example. Each cell in the root directory has a pointer to the corresponding entries in the subdirectory, which have their own scales in turn.

4.1.4 The Twin Grid File [Hutflesz et al. 1988b]. The twin grid file tries to increase space utilization compared to 

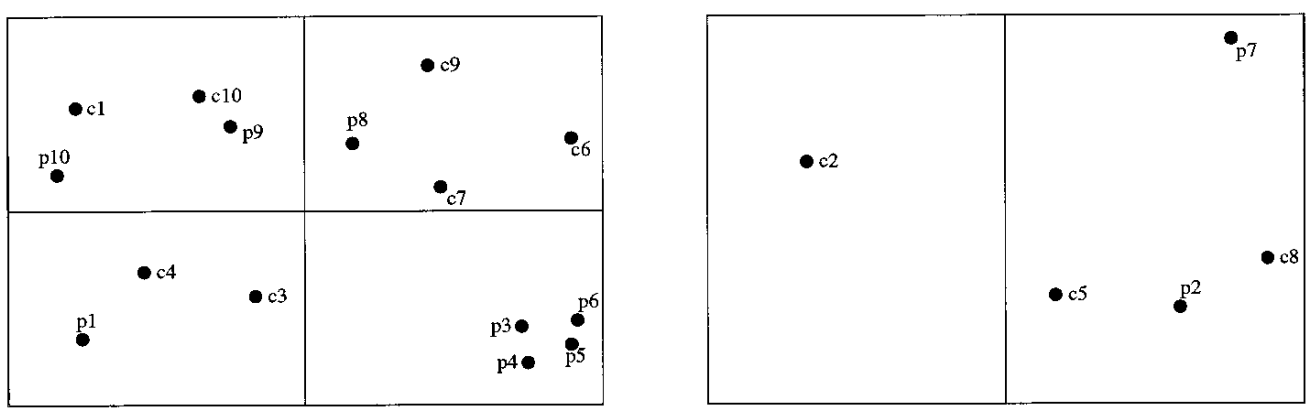

Figure 18. Twin grid file.

the original grid file by introducing a second grid file. As indicated by the name "twin," the relationship between these two grid files is not hierarchical, as in the case of the two-level grid file, but somewhat more balanced. Both grid files span the whole universe. The distribution of the data among the two files is performed dynamically. Hutflesz et al. [1988b] report an average occupancy of $90 \%$ for the twin grid file (compared to $69 \%$ for the original grid file) without substantial performance penalties.

To illustrate the underlying technique, consider the running example depicted in Figure 18. Let us assume that each bucket can accommodate four points. If the number of points in a bucket exceeds that limit, one possibility is to create a new bucket and redistribute the points among the two new buckets. Before doing this, however, the twin grid file tries to redistribute the points between the two grid files. A transfer of points from the primary file $\mathrm{P}$ to the secondary file $\mathrm{S}$ may lead to a bucket overflow in S. It may, however, also imply a bucket underflow in $\mathrm{P}$, which may in turn lead to a bucket merge and therefore to a reduction of buckets in P. The overall objective of the reshuffling is to minimize the total number of buckets in the two grid files $\mathrm{P}$ and $\mathrm{S}$. Therefore we shift points from $P$ to $S$ if and only if the resulting decrease in the number of buckets in $\mathrm{P}$ outweighs the increase in the number of buckets in $\mathrm{S}$. This strategy also favors points to be placed in the primary file in order to form large and empty buckets in the secondary file. Consequently, all points in $\mathrm{S}$ can be associated with an empty or a full bucket region of $P$. Note that there usually exists no unique optimum for the distribution of data points between the two files.

The fact that data points may be found in either of the two grid files requires search operations to visit the two files, which causes some overhead. Nevertheless, the performance results reported by Hutflesz et al. [1988b] indicate that the search efficiency of the twin grid file is competitive with the original grid file. Although the twin grid file is somewhat inferior to the original grid file for smaller query ranges, this changes for larger search spaces.

4.1.5 Multidimensional Linear Hashing. Unlike multidimensional extendible hashing, multidimensional linear hashing uses no or only a very small directory. It therefore occupies relatively little storage compared to extendible hashing, and it is usually possible to keep all relevant information in main memory.

Several different strategies have been proposed to perform the required address computation. Early proposals [Ouksel and Scheuermann 1983] failed to support range queries; however, Kriegel and Seeger [1986] later proposed a variant of linear hashing called multidimensional order-preserving linear hashing with partial expansions (MOLHPE). This structure is based on the idea of partially extending the buckets without expanding the file size at the same 
time. To this end, they use a d-dimensional expansion pointer referring to the group of pages to be expanded next. With this strategy, Kriegel and Seeger can guarantee a modest file growth, at least in the case of well-behaved data. According to their experimental results, MOLHPE outperforms its competitors for uniformly distributed data. It fails, however, for nonuniform distributions, mostly because the hashing function does not adapt gracefully to the given distribution.

To solve this problem, the same authors later applied a stochastic technique [Burkhard 1984] to determine the split points. Because of the name of that technique ( $\alpha$-quantiles), the access method was called quantile hashing [Kriegel and Seeger 1987, 1989]. The critical property of the division in quantile hashing is that the original data, which may have a nonuniform distribution, are transformed into uniformly distributed values for $\alpha$. These values are then used as input to the MOLHPE algorithms for retrieval and update. Since the region boundaries are not necessarily simple binary intervals, a small directory is needed. In exchange, skewed input data can be maintained as efficiently as uniformly distributed data. Piecewise linear order-preserving (PLOP) hashing was proposed by the same authors a year later [Kriegel and Seeger 1988]. Because this structure can also be used as an access method for extended objects, we delay its discussion until Section 5.2.7.

Another variant with better orderpreserving properties than MOLHPE has been reported by Hutflesz et al. [1988a]. Their dynamic z-hashing uses a space-filling technique called z-ordering [Orenstein and Merrett 1984] to guarantee that points located close to each other are also stored close together on the disk. Z-ordering is described in detail in Section 5.1.2. One disadvantage of z-hashing is that a number of useless data blocks will be generated, as in the interpolation-based grid file [Ouksel 1985]. On the other hand, z-hashing lets three to four buckets be read in a row on the average before a seek is required, whereas MOLHPE manages to read only one [Hutflesz et al. 1988a]. Widmayer [1991] later noted, however, that both z-hashing and MOLHPE are of limited use in practice, due to their inability to adapt to different data distributions.

\subsection{Hierarchical Access Methods}

In this section we discuss several PAMs that are based on a binary or multiway tree structure. Except for the BANG file and the buddy tree, which are hybrid structures, they perform no address computation. Like hashing-based methods, however, they organize the data points in a number of buckets. Each bucket usually corresponds to a leaf node of the tree (also called data node) and a disk page, which contains those points located in the corresponding bucket region. The interior nodes of the tree (also called index nodes) are used to guide the search; each of them typically corresponds to a larger subspace of the universe that contains all bucket regions in the subtree below. A search operation is then performed by a topdown tree traversal.

At this point, individual tree structures still dominate the field, although more generic concepts are gradually attracting more attention. The generalized search (GIST) tree by Hellerstein et al. [1995], for example, attempts to subsume many of these common features under a generic architecture.

Differences among individual structures are mainly based on the characteristics of the regions. Table 1 shows that in most PAMs the regions at the same tree level form a partitioning of the universe; that is, they are mutually disjoint, with their union being the complete space. For SAMs this is not necessarily true; as we show in Section 5, overlapping regions and partial coverage are important techniques to improve the search performance of SAMs. 


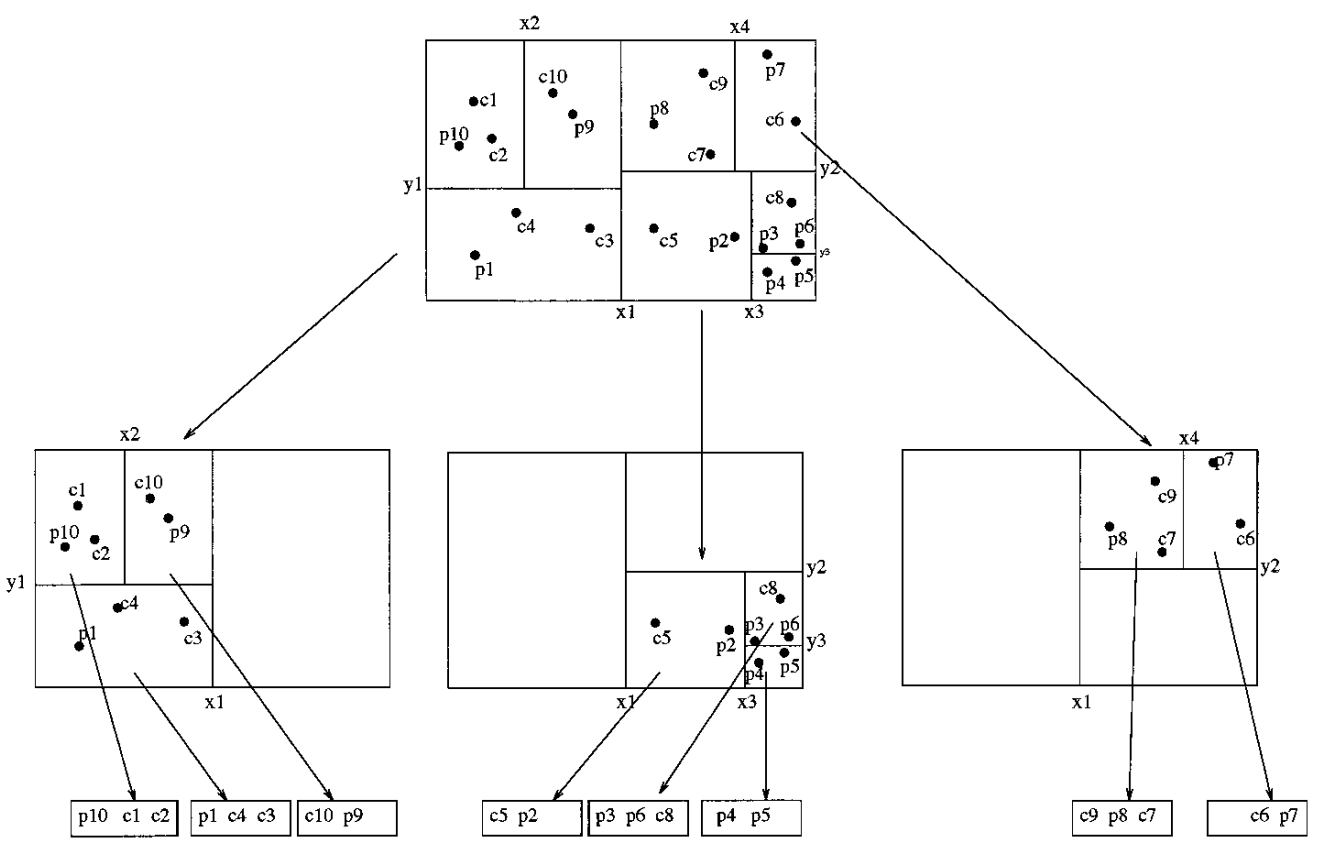

Figure 19. k-d-B-tree.

4.2.1 The k-d-B-Tree [Robinson 1981]. The k-d-B-tree combines some of the properties of the adaptive k-d-tree [Bentley and Friedman 1979] and the B-tree [Comer 1979] to handle multidimensional points. It partitions the universe in the manner of an adaptive k-dtree and associates the resulting subspaces with tree nodes. Each interior node corresponds to an intervalshaped region. Regions corresponding to nodes at the same tree level are mutually disjoint; their union is the complete universe. The leaf nodes store the data points that are located in the corresponding partition. Like the B-tree, the $\mathrm{k}$-d-B-tree is a perfectly balanced tree that adapts well to the distribution of the data. Other than for B-trees, however, no minimum space utilization can be guaranteed. A k-d-B-tree for the running example is sketched in Figure 19.

Search queries are answered in a straightforward manner, analogously to the k-d-tree algorithms. For the insertion of a new data point, one first performs a point search to locate the right bucket. If it is not full, the entry is inserted. Otherwise, it is split and about half the entries are shifted to the new data node. Various heuristics are available to find an optimal split [Robinson 1981]. If the parent index node does not have enough space left to accommodate the new entries, a new page is allocated and the index node is split by a hyperplane. The entries are distributed among the two pages depending on their position relative to the splitting hyperplane, and the split is propagated up the tree. The split of the index node may also affect regions at lower levels of the tree, which must be split by this hyperplane as well. Because of this forced split effect, it is not possible to guarantee a minimum storage utilization.

Deletion is straightforward. After performing an exact match query, the entry is removed. If the number of entries drops below a given threshold, the data node may be merged with a sibling data node as long as the union remains a d-dimensional interval. The procedure to find a suitable sibling node to merge with may involve several nodes. The union of data pages results in the deletion of at least one hyperplane in the 

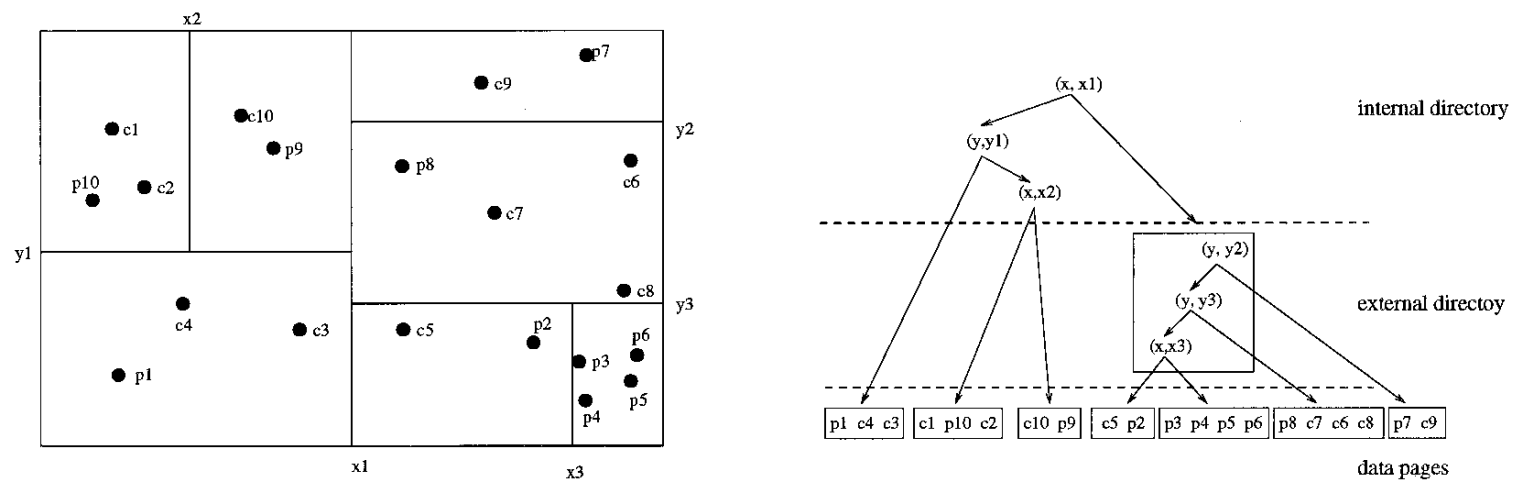

Figure 20. LSD-tree.

parent index node. If an underflow occurs, the deletion has to be propagated up the tree.

4.2.2 The LSD-Tree [Henrich et al. 1989]. We list the LSD (Local Split Decision) tree as a point access method although its inventors emphasize that the structure can also be used for managing extended objects. This claim is based on the fact that the LSD-tree adapts well to data that are nonuniformly distributed and that it is therefore well-suited for use in connection with the transformation technique; a more detailed discussion of this approach appears in Section 5.1.1.

The directory of the LSD-tree is organized as an adaptive k-d-tree, partitioning the universe into disjoint cells of various sizes. This results in a better adaption to the data distribution than the fixed binary partitioning. Although the k-d-tree may be arbitrarily unbalanced, the LSD-tree preserves the external balancing property; that is, the heights of its external subtrees differ at most by one. This property is maintained by a special paging algorithm. If the structure becomes too large to fit in main memory, this algorithm identifies subtrees that can be paged out such that the external balancing property is preserved. Although efficient, this special paging strategy is obviously a major impediment for the integration of the LSD-tree into a general-purpose database system. Figure 20 shows an LSD- tree for the running example with one external directory page.

As indicated previously, the split strategy of the LSD-tree does not assume the data to be uniformly distributed. On the contrary, it tries to accommodate skewed data by combining two split strategies:

- data-dependent $\left(\mathrm{SP}_{1}\right)$ : The choice of the split depends on the data and tries to achieve a most balanced structure; that is, there should be an equal number of objects on both sides of the split. As the name of the structure suggests, this split decision is made locally.

-distribution-dependent $\left(\mathrm{SP}_{2}\right)$ : The split is done at a fixed dimension and position. The given data are not taken into account because an underlying (known) distribution is assumed.

To determine the split position SP, one computes the linear combination of the split locations that would result from applying just one of those strategies:

$$
\mathrm{SP}=\alpha \mathrm{SP}_{1}+(1-\alpha) \mathrm{SP}_{2} .
$$

The factor $\alpha$ is determined empirically based on the given data; it can vary as objects are inserted and deleted from the tree.

Henrich [1995] presented two algorithms to improve the storage utilization of the LSD-tree by redistributing data entries among buckets. Since these 
strategies make the LSD-tree sensitive to the insertion sequence, the splitting strategy must be adapted accordingly. In order to improve the search performance for nonpoint data and range queries, Henrich and Möller [1995] suggest storing auxiliary information on the existing data regions along with the index entries of the LSD-tree.

4.2.3 The Buddy Tree [Seeger and Kriegel 1990]. The buddy tree is a dynamic hashing scheme with a treestructured directory. The tree is constructed by consecutive insertion, cutting the universe recursively into two parts of equal size with iso-oriented hyperplanes. Each interior node $\nu$ corresponds to a d-dimensional partition $\mathrm{P}^{\mathrm{d}}(\nu)$ and to an interval $\mathrm{I}^{\mathrm{d}}(\nu) \subseteq \mathrm{P}^{\mathrm{d}}(\nu)$. $\mathrm{I}^{\mathrm{d}}(\nu)$ is the MBB of the points or intervals below $\nu$. Partitions $\mathrm{P}^{\mathrm{d}}$ (and therefore intervals $\mathrm{I}^{\mathrm{d}}$ ) that correspond to nodes on the same tree level are mutually disjoint. As in all tree-based structures, the leaves of the directory point to the data pages. Other important properties of the buddy tree include:

(1) each directory node contains at least two entries;

(2) whenever a node $\nu$ is split, the MBBs $\mathrm{I}^{\mathrm{d}}\left(\nu_{\mathrm{i}}\right)$ and $\mathrm{I}^{\mathrm{d}}\left(\nu_{\mathrm{j}}\right)$ of the two resulting subnodes $\nu_{\mathrm{i}}$ and $\nu_{\mathrm{j}}$ are recomputed to reflect the current situation; and

(3) except for the root of the directory, there is exactly one pointer referring to each directory page.

Due to property 1 , the buddy tree may not be balanced; that is, the leaves of the directory may be on different levels. Property 2 tries to achieve a high selectivity at the directory level. Properties 1 and 3 make sure that the growth of the directory remains linear. To avoid the deadlock problem of the grid file, the buddy tree uses k-d-trees [Orenstein 1982] to partition the universe. Only a restricted number of buddies are admitted, namely, those that could have been obtained by some recursive halving of the universe. However, as shown by
Seeger and Kriegel [1990], the number of possible buddies is larger than in the grid file and other structures, which makes the buddy tree more flexible in the case of updates. Experiments by Kriegel et al. [1990] indicate that the buddy tree is superior to several other PAMs, including the hB-tree, the BANG file, and the two-level grid file. A buddy tree for the running example is shown in Figure 21.

Two older structures, the interpolation-based grid file by Ouksel [1985] and the balanced multidimensional extendible hash tree by Otoo [1986], are both special cases of the buddy tree that can be obtained by restricting the properties of the regions. Interpolationbased grid files avoid the excessive growth of the grid file directory by representing blocks explicitly, which guarantees that there is only one directory entry for each data bucket. The disadvantage of this approach is that empty regions have to be introduced in the case of skewed data input. Seeger [1991] later showed that the buddy tree can easily be modified to handle spatially extended objects by using one of the techniques presented in Section 5.

4.2.4 The BANG File [Freeston 1987]. To obtain a better adaption to the given data points, Freeston [1987] proposed a new structure, which he called the BANG (Balanced And Nested Grid) file-even though it differs from the grid file in many aspects. Similar to the grid file, it partitions the universe into intervals (boxes). What is different, however, is that in the BANG file bucket regions may intersect, which is not possible in the regular grid file. In particular, one can form nonrectangular bucket regions by taking the geometric difference of two or more intervals (nesting). To increase storage utilization, it is possible during insertion to redistribute points between different buckets. To manage the directory, the BANG file uses a balanced search tree structure. In combination with the hash-based partitioning of the universe, 


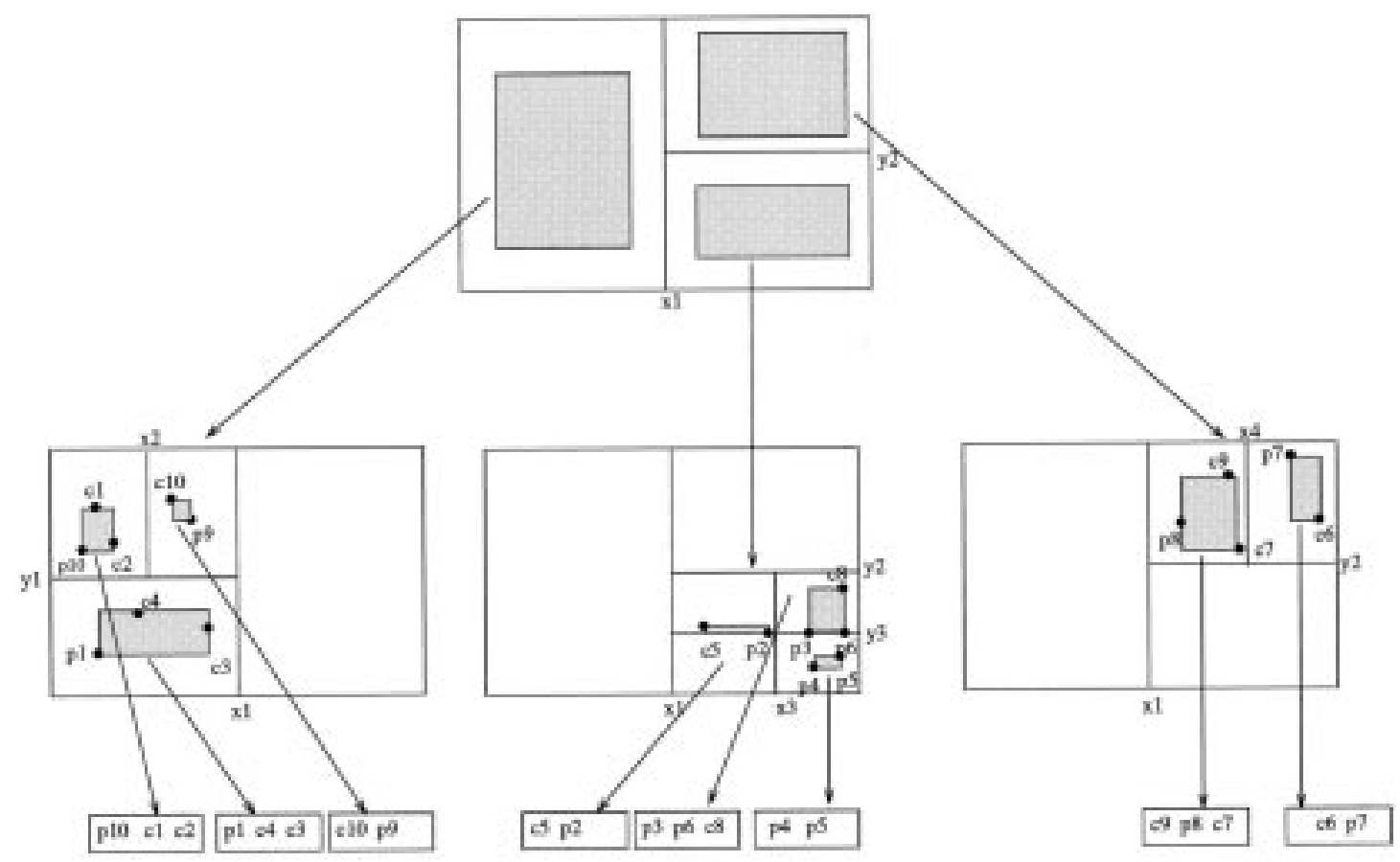

Figure 21. Buddy tree.

the BANG file can therefore be viewed as a hybrid structure.

Figure 22 shows the BANG file for the running example. Three rectangles have been cut out of the universe R1: R2, R5, and R6. In turn, the rectangles R3 and R4 are nested into R2 and R5, respectively. If one represents the resulting space partitioning as a tree using bit interleaving, one obtains the structure shown on the right-hand side of Figure 22. Here the asterisk represents the empty string, that is, the universe. A comparison with Figure 13 shows that the BANG file can in fact be regarded as a paginated version of the BD-tree discussed in Section 3.2.3.

In order to achieve a high storage utilization, the BANG file performs spanning splits that may lead to the displacement of parts of the tree. As a result, a point search may in the worst case require the traversal of the entire directory in a depth-first manner. To address this problem, Freeston [1989a] later proposed different splitting strategies, including forced splits as used by the k-d-B-tree. These strategies avoid the spanning problem at the possible expense of lower storage utilization. Kumar [1994a] made a similar proposal based on the BD-tree and called the resulting structure a G-tree (grid tree). The structure differs from the BD-tree in the way the partitions are mapped into buckets. To obtain a simpler mapping, the G-tree sacrifices the minimum storage utilization that holds for the BD-tree.

Although the data partitioning given in Figure 22 is feasible for the BD-tree and the original BANG file, it cannot be achieved with the BANG file using forced splits [Freeston 1989a]. For this variant, we would have to split the root and move, for example, entry c5 to the bucket containing the entries $\mathrm{p} 7$ and $c 6$.

Freeston [1989b] also proposed an extension to the BANG file to handle extended objects. As often found in PAM extensions, the centroid is used to determine the bucket in which to place a given object. To account for the object's spatial extension, the bucket regions are extended where necessary [Seeger and Kriegel 1988; Ooi 1990]. 

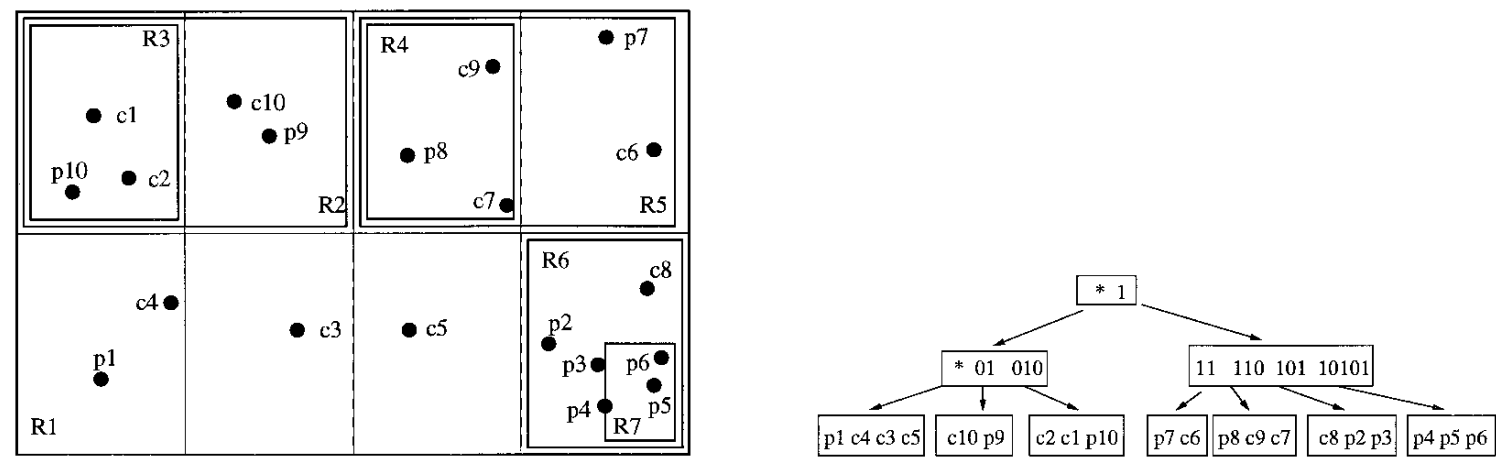

Figure 22. BANG file.

Ouksel and Mayer [1992] proposed an access method called a nested interpolation-based grid file that is closely related to the BANG file. The major difference concerns the way the directory is organized. In essence, the directory consists of a list of one-dimensional access methods (e.g., B-trees) storing the z-order encoding of the different data regions, along with pointers to the respective data buckets. By doing so, Ouksel and Mayer improved the worst-case bounds from $\mathrm{O}(\mathrm{n})$ (as in the case of the BANG file) to $O\left(\log _{b} n\right)$, where $b$ is bucket size.

4.2.5 The hB-Tree [Lomet and Salzberg 1989, 1990]. The hB-tree (holey brick tree) is related to the k-d-B-tree in that it utilizes $\mathrm{k}$-d-trees to organize the space represented by its interior nodes. One of the most noteworthy differences is that node splitting is based on multiple attributes. As a result, nodes no longer correspond to d-dimensional intervals but to intervals from which smaller intervals have been excised. Similar to the BANG file, the result is a somewhat fractal structure (a holey brick) with an external enclosing region and several cavities called extracted regions. As we show later, this technique avoids the cascading of splits that is typical for many other structures.

In order to minimize redundancy, the k-d-tree corresponding to an interior node can have several leaves pointing to the same child node. Strictly speaking, the hB-tree is therefore no longer a tree but a directed acyclic graph. With regard to the geometry, this corresponds to the union of the corresponding regions. Once again, the resulting region is typically no longer box-shaped. This peculiarity is illustrated in Figure 23, which shows an hB-tree for the running example. Here the root node contains two pointers to its left descendant node. Its corresponding region $u$ is the union of two rectangles: the one to the left of $x 1$ and the one above $y 1$. The remaining space (the right lower quadrant) is excluded from $u$, which is made explicit by the entry ext in the corresponding k-dtree. A similar observation applies to region G, which is again L-shaped: it corresponds to the NW, the SE, and the NE quadrants of the rectangle above $y 1$.

Searching is similar to the k-d-B-tree; each internal $\mathrm{k}$-d-tree is traversed as usual. Insertions are also carried out analogously to the k-d-B-tree until a leaf node reaches its capacity and a split is required. Instead of using just one single hyperplane to split the node, the $\mathrm{hB}$-tree split is based on more than one attribute and on the internal k-d-tree of the data node to be split. Lomet and Salzberg [1989] show that this policy guarantees a worst-case data distribution between the two resulting two nodes of $\frac{1}{3}: \frac{2}{3}$. This observation is not restricted to the hB-tree but generalizes to other access methods such as the BD-tree and the BANG file.

The split of the leaf node causes the introduction of an additional k-d-tree 

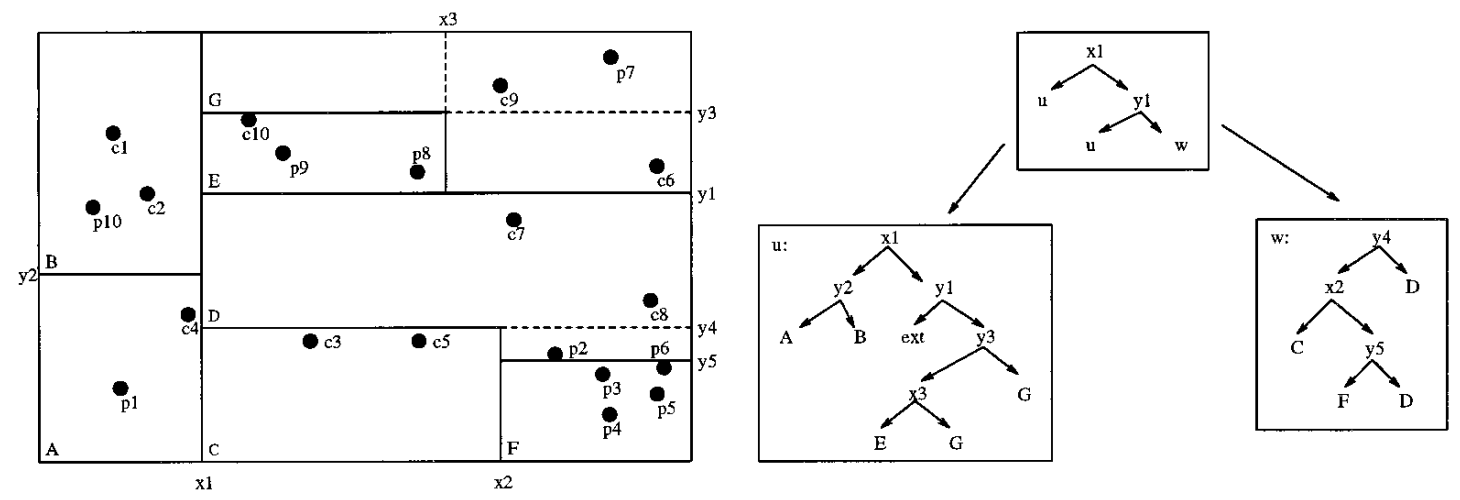

Figure 23. hB-tree.

node to describe the resulting subspace. This may in turn lead to the split of the ancestor node and its k-d-tree. Since k-d-trees are not height-balanced, splitting the tree at its root may lead to an unbalanced distribution of the nodes. The tree is therefore usually split at a lower level, which corresponds to the excision of a convex region from the space corresponding to the node to be split. The entries belonging to that subspace are extracted and moved to a new hB-tree node. To reflect the absence of the excised region, the hB-tree node is assigned an external marker, which indicates that the region is no longer a simple interval. With this technique the problem of forced splits is avoided. Splits are local and do not have to be propagated downwards.

In summary, the leaf nodes of the internal k-d-trees are used to

-reference a collection of data records; - reference other hB-tree nodes;

-indicate that a part of this tree has been extracted.

In a later Ph.D. thesis [Evangelidis 1994], the hB-tree is extended to allow for concurrency and recovery by modifying it in such a way that it becomes a special case of the П-tree [Lomet and Salzberg 1992]. Consequently, the new structure is called the $\mathrm{hB}^{\mathrm{II}}$-tree [Evangelidis et al. 1995]. As a result of these modifications, the new structure can immediately take advantage of the
II-tree node consolidation algorithm. The lack of such an algorithm has been one of the major weaknesses of the hBtree. Furthermore, the $\mathrm{hB}^{\Pi}$-tree corrects a flaw in the splitting/posting algorithm of the hB-tree that may occur for more than three index levels. The essential idea of the correction is to impose restrictions on the splitting/ posting algorithms, which in turn affects the space occupancy.

One minor problem remains: as mentioned, the hB-tree may store several references to the same child node. The number of nodes may in principle expose a growth behavior that is superlinear in the number of regions; however, this observation seems of mainly theoretical interest. According to the authors of the $\mathrm{hB}^{\Pi}$-tree [Evangelidis et al. 1995], it is quite rare that more than one leaf of the underlying k-d tree refers to any given child. In their experiments, more than $95 \%$ of the index nodes and all of the data nodes had only one such reference.

4.2.6 The BV-Tree [Freeston 1995]. The BV-tree represents an attempt to solve the d-dimensional B-tree problem, that is, to find a generic generalization of the B-tree to higher dimensions. The BV-tree is not meant to be a concrete access method, but rather a conceptual framework that can be applied to a variety of existing access methods, including the BANG file or the hB-tree.

Freeston's proposal is based on the 

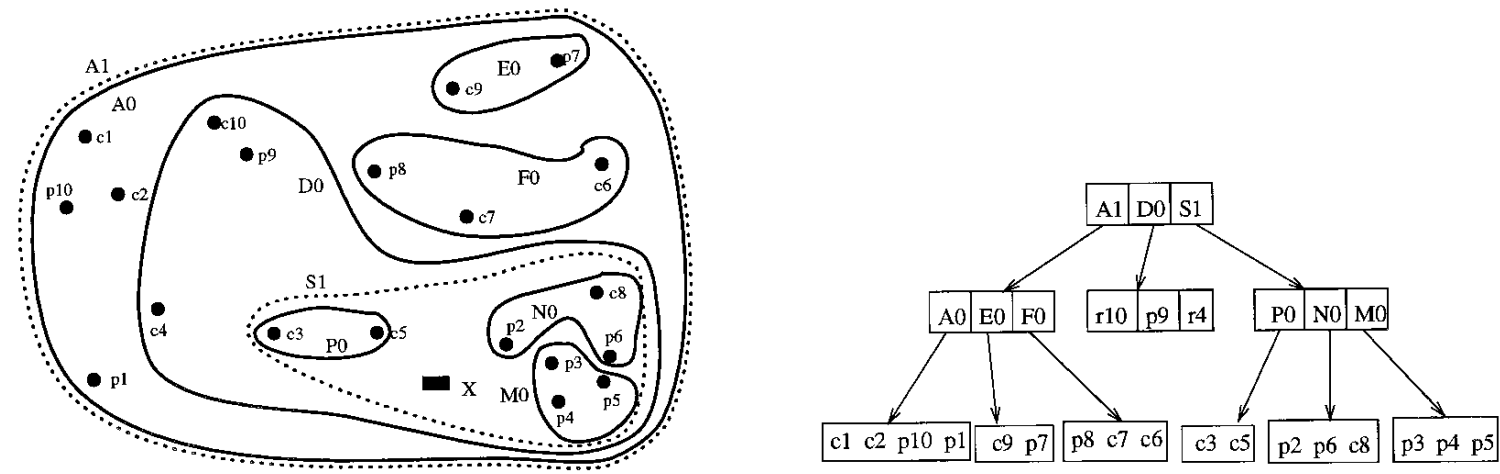

Figure 24. BV-tree.

conjecture that one can maintain the major strengths of the B-tree in higher dimensions, provided one relaxes the strict requirements concerning tree balance and storage utilization. The BVtree is not completely balanced. Furthermore, although the B-tree guarantees a worst-case storage utilization of $50 \%$, Freeston argues that such a comparatively high storage utilization cannot be ensured for higher dimensions for topological reasons. However, the BV-tree manages to achieve the 33\% lower bound suggested by Lomet and Salzberg [1989].

To achieve a guaranteed worst-case search performance, the BV-tree combines the excision concept [Freeston 1987] with a technique called promotion. Here, intervals from lower levels of the tree are moved up the tree, that is, closer to the root. To keep track of the resulting changes, with each promoted region we store a level number (called a guard) that denotes the region's original level.

The search algorithms are based on a notional backtracking technique. While descending the tree, we store possible alternatives (relevant guards of the different index levels) in a guard set. The entries of this set act as backtracking points and represent a single path from the root to the level currently inspected; for point queries, they can be maintained as a stack. To answer a point query, we start at the root and inspect all node entries to see whether the cor- responding regions overlap the search point. Among those entries inspected, we choose the best-matching entry to investigate next. We may possibly also store some guards in the guard set. At the next level this procedure is repeated recursively, this time taking the stored guards into account. Before following the best-matching entry down to the next level, the guard set is updated by merging the matching new guards with the existing ones. Two guards at the same level are merged by discarding the poorer match. This search continues recursively until we reach the leaf level. Note that for point queries, the length of the search path is equal to the height of the BV-tree because each region in space is represented by a unique node entry.

Figure 24 shows a BV-tree and the corresponding space partitioning for the running example. For illustration purposes we confine the grouped regions or objects not by a tight polyline, but by a loosely wrapped boundary. In this example, the region D0 acts as a guard. It is clear from the space partitioning that D0 originally belongs to the bottom index level (i.e., the middle level in the figure). Since it functions as a guard for the enclosed region S1, however, it has been promoted to the root level. Suppose we are interested in all objects intersecting the black rectangle $X$. Starting at the root, we place D0 in the guard set and investigate S1. Because inspection of S1 reveals that the search 
region is included neither in $\mathrm{PO}$ nor in $\mathrm{NO}$ or MO, we backtrack to DO and inspect the entries for D0. In our example, no entry satisfies the query.

In a later paper, Freeston [1997] discusses complexity issues related to updates of guards. In the presence of such updates, it is necessary to "downgrade" (demote) entries that are no longer guards, which may in turn affect the overall structure negatively. Freeston's conclusion is that the logarithmic access performance and the minimum storage utilization of the BV-tree can be preserved by postponing the demotion of such entries, which may lead to (very) large index nodes.

\subsection{Space-Filling Curves for Point Data}

We al ready mentioned the main reason why the design of multidimensional access methods is so difficult compared to the one-dimensional case: There is no total order that preserves spatial proximity. One way out of this dilemma is to find heuristic solutions, that is, to look for total orders that preserve spatial proximity at least to some extent. The idea is that if two objects are located close together in original space, there should at least be a high probability that they are close together in the total order, that is, in the one-dimensional image space. For the organization of this total order one could then use a one-dimensional access method (such as a $\mathrm{B}^{+}$-tree), which may provide good performance at least for point queries. Range queries are somewhat more complicated; a simple mapping from multidimensional to one-dimensional range queries often implies major performance penalties. Tropf and Herzog [1981] present a more sophisticated and efficient algorithm for this problem.

Research on the underlying mapping problem goes back well into the last century; see Sagan [1994] for a survey. With regard to its relevance for spatial searching, Samet [1990b] provides a good overview of the subject. One thing all proposals have in common is that they first partition the universe with a grid. Each of the grid cells is labeled with a unique number that defines its position in the total order (the spacefilling curve). The points in the given data set are then sorted and indexed according to the grid cell in which they are contained. Note that although the labeling is independent of the given data, it is obviously critical for the preservation of proximity in one-dimensional address space. That is, the way we label the cells determines how clustered adjacent cells are stored on secondary memory.

Figure 25 shows four common labelings. Figure 25a corresponds to a rowwise enumeration of the cells [Samet 1990b]. Figure 25b shows the cell enumeration imposed by the Peano curve [Morton 1966], also called quad codes [Finkel and Bentley 1974], N-trees [White 1981], locational codes [Abel and Smith 1983], or z-ordering [Orenstein and Merrett 1984]. Figure 25c shows the Hilbert curve [Faloutsos and Roseman 1989; J agadish 1990a], and Figure 25d depicts Gray ordering [Faloutsos 1986, 1988], which is obtained by interleaving the Gray codes of the $x$ - and $y$-coordinates in a bitwise manner. Gray codes of successive cells differ in exactly one bit.

Based on several experiments, Abel and Mark [1990] conclude that z-ordering and the Hilbert curve are most suitable as multidimensional access methods. J agadish [1990a] and Faloutsos and Rong [1991] all prefer the Hilbert curve of those two.

Z-ordering is one of the few spatial access methods that has found its way into commercial database products. In particular, Oracle [1995] has adapted the technique and offered it for some time as a product.

An important advantage of all spacefilling curves is that they are practically insensitive to the number of dimensions if the one-dimensional keys can be arbitrarily large. Everything is mapped into one-dimensional space, and one's favorite one-dimensional access method can 

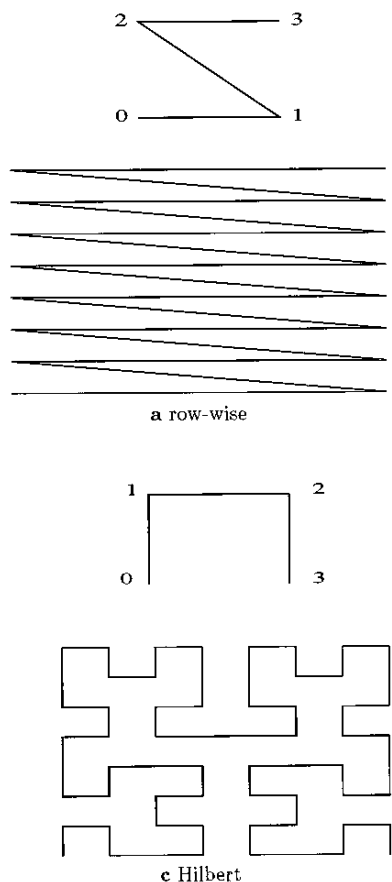
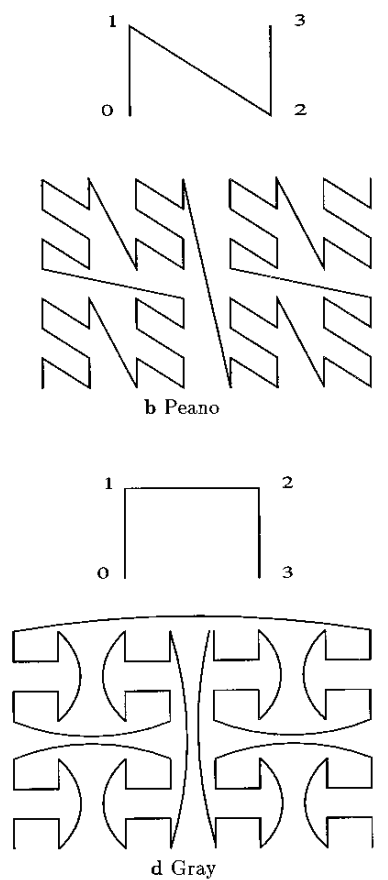

Figure 25. Four space-filling curves.

be applied to manage the data. An obvious disadvantage of space-filling curves is that incompatible index partitions cannot be joined without recomputing the codes of at least one of the two indices.

\section{SPATIAL ACCESS METHODS}

All multidimensional access methods presented in the previous section have been designed to handle sets of data points and support spatial searches on them. None of those methods is directly applicable to databases containing objects with a spatial extension. Typical examples include geographic databases, containing mostly polygons, or mechanical CAD data, consisting of three-dimensional polyhedra. In order to handle such extended objects, point access methods have been modified using one of the techniques:

(1) transformation (object mapping),

(2) overlapping regions (object bounding),

(3) clipping (object duplication), or

(4) multiple layers.
A simpler version of this classification was first introduced by Seeger and Kriegel [1988]. Later on, Kriegel et al. [1991] added another dimension to this taxonomy: a spatial access method's base type, that is, the spatial data type it supports primarily. Table 2 shows the resulting classification of spatial access methods. Note that most structures use the interval as a base type.

In the following sections, we present each of these techniques in detail, together with several SAMs based on it.

\subsection{Transformation}

One-dimensional access methods (Section 3.1) and PAMs (Section 4) can often be used to manage spatially extended objects, provided the objects are first transformed into a different representation. There are essentially two options: one can either transform each object into a higher-dimensional point [Hinrichs 1985; Seeger and Kriegel 1988], or transform it into a set of one-dimensional intervals by means of space-filling curves. We discuss the two techniques in turn. 
Table 2. Classification of SAMs [Kriegel et al. 1991]

\begin{tabular}{|c|c|c|c|c|}
\hline \multirow[t]{2}{*}{ technique } & \multicolumn{4}{|c|}{ base type } \\
\hline & grid cell & interval (box) & sphere & polyhedron \\
\hline transformation & $\begin{array}{l}\text { zkdB }{ }^{+} \text {-tree [Oren- } \\
\text { stein 1986], } \\
\text { BANG file [Free- } \\
\text { ston 1987], hB- } \\
\text { tree [Lomet and } \\
\text { Salzberg 1989] }\end{array}$ & $\begin{array}{l}\text { all PAMs described in Section } 4 \text { ex- } \\
\text { cept of the BANG file and the hB- } \\
\text { tree }\end{array}$ & & $\begin{array}{l}\text { P-tree [Jagadish } \\
\text { 1990b] }\end{array}$ \\
\hline $\begin{array}{l}\text { overlapping } \\
\text { regions }\end{array}$ & & $\begin{array}{l}\text { R-tree [Guttman 1984], R } \text { R }^{*} \text {-tree } \\
\text { [Beckmann et al. 1990], skd-tree } \\
\text { [Ooi et al. 1987], GBD-tree [Ohsawa } \\
\text { and Sakauchi 1990], Hilbert R-tree } \\
\text { [Kamel and Faloutsos 1994], buddy } \\
\text { tree with overlapping [Seeger 1991] }\end{array}$ & $\begin{array}{l}\text { sphere tree } \\
\text { [Oosterom 1990] }\end{array}$ & $\begin{array}{l}\text { P-tree } \\
\text { [Schiwietz 1993], } \\
\text { KD2B-tree [Oos- } \\
\text { terom 1990] }\end{array}$ \\
\hline clipping & & $\begin{array}{l}\text { EXCELL [Tamminen 1982], ex- } \\
\text { tended k-d-tree [Matsuyama et al. } \\
\text { 1984], R+-tree [Sellis et al. 1987], } \\
\text { buddy tree with clipping [Seeger } \\
\text { 1991] }\end{array}$ & & $\begin{array}{l}\text { cell tree [Günther } \\
\text { 1988] }\end{array}$ \\
\hline multiple layers & & $\begin{array}{l}\text { multi-layer grid file [Six and Wid- } \\
\text { mayer 1988], R-file [Hutflesz et al. } \\
\text { 1990] }\end{array}$ & & \\
\hline
\end{tabular}

5.1.1 Mapping to Higher-Dimensi onal Space. Simple geometric shapes can be represented as points in higher-dimensional space. For example, it takes four real numbers to represent a (two-dimensional) rectangle in $\mathrm{E}^{2}$. Those numbers may be interpreted as coordinates of a point in $E^{4}$. One possibility is to take the $x$ - and $y$-coordinates of two diagonal corners (endpoint transformation); another option is based on the centroid and two additional parameters for the extension of the object in the $\mathrm{x}$ - and $y$-direction (midpoint transformation). Any such transformation maps a database of rectangles onto a database of four-dimensional points, which can then be managed by one of the PAMs discussed in the previous section. Search operations can be expressed as point and region queries in this dual space.

If the original database contains more complex objects, they have to be approximated-for example, by a rectangle or a sphere-before transformation. In this case, the point access method can lead to only a partial solution (cf. Figure 2).

Figures 26 and 27 show the dual space equivalents of some common queries. Figure 26 uses the endpoint transformation and Figure 27 the midpoint transformation. For presentation purposes, the figure shows a mapping from intervals in $E^{1}$ to points in $E^{2}$. Figures $26 a$ and $27 a$ show the transformation result for the range query with search range $[I, u]$. In dual space this range query maps into a general region query. Any point in dual space that lies in the shaded areas corresponds to an interval in original space that overlaps the search interval $[\mathrm{I}, \mathrm{u}]$, and vice versa. Enclosure and containment queries with the interval $[I, u]$ as argument also map into general region queries (Figures 26b and 27b). A point query, finally, maps into a range query for the endpoint transformation (Fig. 26c) and a general region query for the midpoint transformation (Fig. 27c).

Notwithstanding its conceptual elegance, this approach has several disadvantages. First, as the preceding examples indicate, the formulation of point and range queries in dual space is usually much more complicated than in original space [Nievergelt and Hinrichs 1987]. Finite search regions may map into infinite search regions in dual space, and some more complex queries involving spatial predicates may no Ionger be expressible at all [Henrich et 

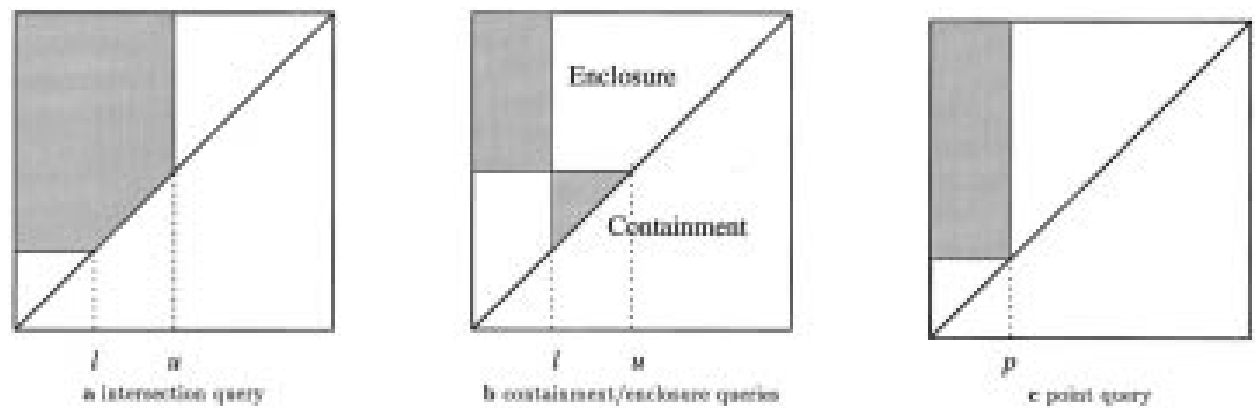

Figure 26. Search queries in dual space-endpoint transformation: (a) intersection query; (b) containment/enclosure queries; (c) point query.
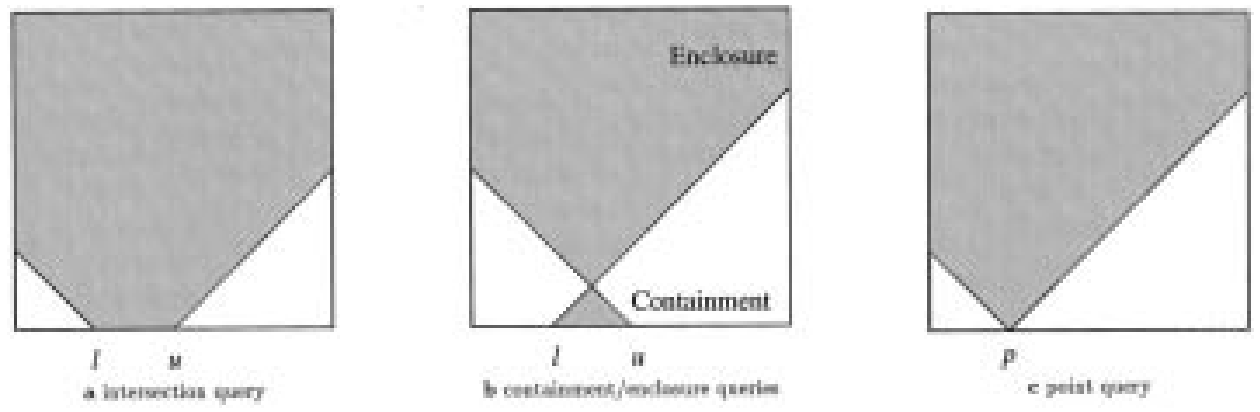

Figure 27. Search queries in dual space-midpoint transformation: (a) intersection query; (b) containment/enclosure queries; (c) point query.

al. 1989; Orenstein 1990; Pagel et al. 1993]. Second, depending on the mapping chosen, the distribution of points in dual space may be highly nonuniform even though the original data are uniformly distributed. With the endpoint transformation, for example, there are no image points below the main diagonal [Faloutsos et al. 1987]. Third, the images of two objects that are close in the original space may be arbitrarily far apart from each other in dual space.

To overcome some of these problems, Henrich et al. [1989], Faloutsos and Rong [1991], as well as Pagel et al. [1993] have proposed special transformation and split strategies. A structure designed explicitly to be used in connection with the transformation technique is the LSD-tree (cf. Section 4.2.2). Performance studies by Henrich and Six [1991] confirm the claim that the LSDtree adapts well to nonuniform distributions, which is of particular relevance in this context. It also contains a mecha- nism to avoid searching large empty query spaces, which may occur as a result of the transformation.

5.1.2 Space-Filling Curves for Extended Objects. Space-filling curves (cf. Section 4.3) are a very different type of transformation approach that seems to have fewer of the drawbacks listed in the previous section. Space-filling curves can be used to represent extended objects by a list of grid cells or, equivalently, a list of one-dimensional intervals that define the position of the grid cells concerned. In other words, a complex spatial object is approximated not by only one simpler object, but by the union of several such objects. There are different variations of this basic concept, including z-ordering [Orenstein and Merrett 1984], the Hilbert R-tree [Kamel and Faloutsos 1994], and the UB-tree [Bayer 1996]. As an example, we discuss z-ordering in more detail. 


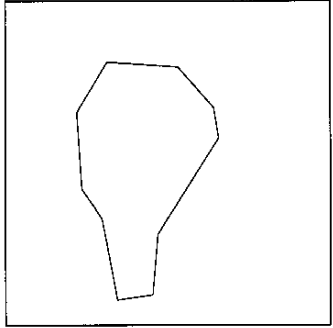

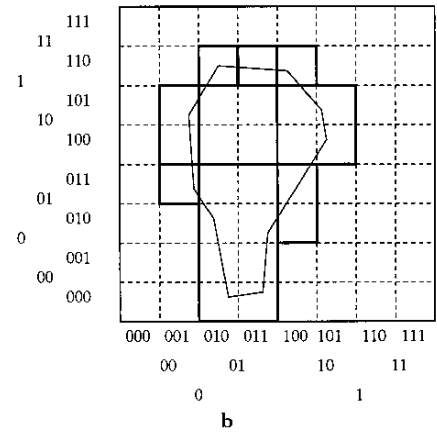

Figure 28. Z-ordering of a polygon.

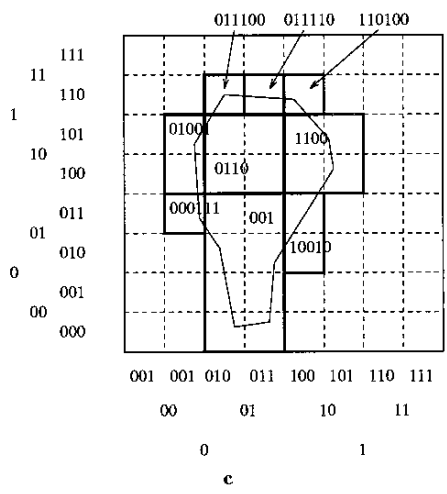

For a discussion of the Hilbert R-tree, see Section 5.2.1.

Z-ordering [Orenstein and Merrett 1984] is based on the Peano curve. A simple algorithm to obtain the z-ordering representation of a given extended object can be described as follows. Starting from the (fixed) universe containing the data object, space is split recursively into two subspaces of equal size by $(d-1)$-dimensional hyperplanes. As in the k-d-tree, the splitting hyperplanes are iso-oriented, and their directions alternate in fixed order among the d possibilities. The subdivision continues until one of the following conditions holds.

(1) The current subspace does not overlap the data object.

(2) The current subspace is fully enclosed in the data object.

(3) Some given level of accuracy has been reached.

The data object is thus represented by a set of cells, called Peano regions or z-regions. As shown in Section 3.2.3, each such Peano region can be represented by a unique bit string, called Peano code, ST_MortonNumber, z-value, or DZ-expression. Using those bit strings, the cells can then be stored in a standard one-dimensional index, such as a $\mathrm{B}^{+}$-tree.

Figure 28 shows a simple example. Figure 28a shows the polygon to be approximated, with the frame represent- ing the universe. After several splits, starting with a vertical split line, we obtain Figure 28b. Nine Peano regions of different shapes and sizes approximate the object. The labeling of each Peano region is shown in Figure 28c. Consider the Peano region $\bar{z}$ in the lower left part of the given polygon. It lies to the left of the first vertical hyperplane and below the first horizontal hyperplane, resulting in the first two bits being 00 . As we further partition the lower left quadrant, z lies on the left of the second vertical hyperplane but above the second horizontal hyperplane. The complete bit string accumulated so far is therefore 0001. In the next round of decompositions, $\bar{z}$ lies to the right of the third vertical hyperplane and above the third horizontal hyperplane, resulting in two additional 1s. The complete bit string describing $\bar{z}$ is therefore 000111.

Figures 28b and $28 \mathrm{c}$ also give some bit strings along the coordinate axes, which describe only the splits orthogonal to the given axis. The string 01 on the x-axis, for example, describes the subspace to the left of the first vertical split and to the right of the second vertical split. By bit-interleaving the bit strings that one finds when projecting a Peano region onto the coordinate axes, we obtain its Peano code. Note that if a Peano code $z_{1}$ is the prefix of some other Peano code $z_{2}$, the Peano region corresponding to $\mathrm{z}_{1}$ encloses the Peano re- 


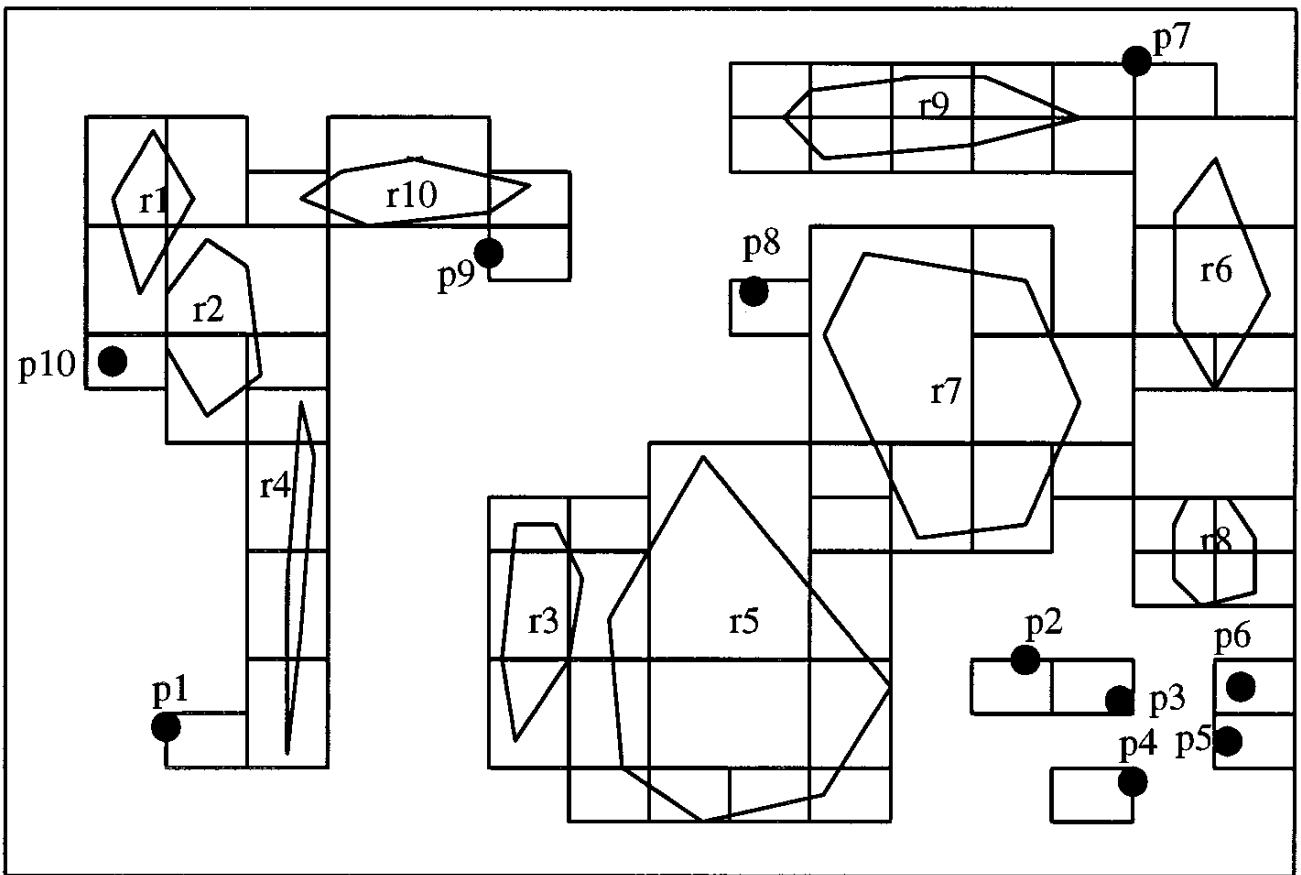

Figure 29. Z-ordering.

gion corresponding to $z_{2}$. The Peano region corresponding to 00 , for example, encloses the regions corresponding to 0001 and 000. This is an important observation, since it can be used for query processing [Gaede and Riekert 1994]. Figure 29 shows Peano regions for the running example.

As z-ordering is based on an underlying grid, the resulting set of Peano regions is usually only an approximation of the original object. The termination criterion depends on the accuracy or granularity (maximum number of bits) desired. More Peano regions obviously yield more accuracy, but they also increase the size and complexity of the approximation. As pointed out by Orenstein [1989b], there are two possibly conflicting objectives: the number of Peano regions to approximate the object should be small, since this results in fewer index entries; and the accuracy of the approximation should be high, since this reduces the expected number of false drops [Orenstein 1989a, b; Gaede 1995b]. Objects are thus paged in from secondary memory, only to find out that they do not satisfy the search predicate. A simple way to reduce the number of false drops is to add a single bit to the encoding that reflects for each Peano region whether it is completely enclosed in the original object [Gaede 1995a]. An advantage of z-ordering is that local changes of granularity lead to only local changes of the corresponding encoding.

\subsection{Overlapping Regions}

The key idea of the overlapping regions technique is to allow different data buckets in an access method to correspond to mutually overlapping subspaces. With this method we can assign any extended object directly and as a whole to one single bucket region. Consider, for instance, the k-d-B-tree for the running example, depicted in Figure 19 , and one of the polygons given in the scenario (Figure 9), say r10. r10 overlaps two bucket regions, the one containing p10, c1, and $c 2$, and the other one containing $\mathrm{c} 10$ and $\mathrm{p9}$. If we extend one of those regions to accommodate r10, this polygon could be stored in the 
corresponding bucket. Note, however, that this extension inevitably leads to an overlap of regions.

Search algorithms can be applied almost unchanged. The only differences are due to the fact that the overlap may increase the number of search paths we have to follow. Even a point query may require the investigation of multiple search paths because there may be several subspaces at any index level that include the search point. For range and region queries, the average number of search paths increases as well.

Hence, although functionality is not a problem when using overlapping regions, performance can be. This is particularly relevant when the spatial database contains objects whose size is large relative to the size of the universe. Typical examples are known from geographic applications where one must represent objects of widely varying size (such as buildings and states) in the same spatial database. Each insertion of a new data object may increase the overlap and therefore the average number of search paths to be traversed per query. Eventually, the overlap between subspaces may become large enough to render the index ineffective because one ends up searching most of the index for each single query. A well-known example where this degenerate behavior has been observed is the R-tree [Guttman 1984; Greene 1989]. Several modifications have been presented to mitigate these problems, including a technique to minimize the overlap [Roussopoul os and Leifker 1985]; see Section 5.2.1 for a detailed discussion.

A minor problem with overlapping regions concerns ambiguities during insertion. If we insert a new object, we could in principle enlarge any subspace to accommodate it. To optimize performance, there exist several strategies [Pagel et al. 1993]. For example, we could try to find the subspace that causes minimal additional overlap, or the one that requires the least enlargement. If it takes too long to compute the optimal strategy for every insertion, some heuristic may be used.

When a subspace needs to be split, one also tries to find a split that leads to minimal overall overlap. Guttman [1984], Greene [1989], and Beckmann et al. [1990] suggest some heuristics for this problem.

5.2.1 The R-Tree [Guttman 1984]. An R-tree corresponds to a hierarchy of nested d-dimensional intervals (boxes). Each node $\nu$ of the R-tree corresponds to a disk page and a d-dimensional interval $\mathrm{I}^{\mathrm{d}}(\nu)$. If $\nu$ is an interior node then the intervals corresponding to the descendants $\nu_{\mathrm{i}}$ of $\nu$ are contained in $\mathrm{I}^{\mathrm{d}}(\nu)$. Intervals at the same tree level may overlap. If $\nu$ is a leaf node, $\mathrm{I}^{\mathrm{d}}(\nu)$ is the d-dimensional minimum bounding box of the objects stored in $\nu$. For each object in turn, $\nu$ stores only its MBB and a reference to the complete object description. Other properties of the R-tree include the following.

- Every node contains between $\mathrm{m}$ and $M$ entries unless it is the root. The lower bound $\mathrm{m}$ prevents the degeneration of trees and ensures an efficient storage utilization. Whenever the number of a node's descendants drops bel ow $\mathrm{m}$, the node is deleted and its descendants are distributed among the sibling nodes (tree condensation). The upper bound $M$ can be derived from the fact that each tree node corresponds to exactly one disk page.

- The root node has at least two entries unless it is a leaf.

-The R-tree is height-balanced; that is, all leaves are at the same level. The height of an R-tree is at most $\left\lceil\log _{m}(N)\right\rceil$ for $N$ index records $(N>1)$.

Searching in the R-tree is similar to the B-tree. At each index node $\nu$, all index entries are tested to see whether they intersect the search interval $I_{s}$. We then visit all child nodes $\nu_{\mathrm{i}}$ with I ${ }^{\mathrm{d}}\left(\nu_{\mathrm{i}}\right) \cap$ $I_{s} \neq \emptyset$. Due to the overlapping region paradigm, there may be several intervals $\mathrm{I}^{\mathrm{d}}\left(\nu_{\mathrm{i}}\right)$ that satisfy the search predicate. In the worst case, one may have to 

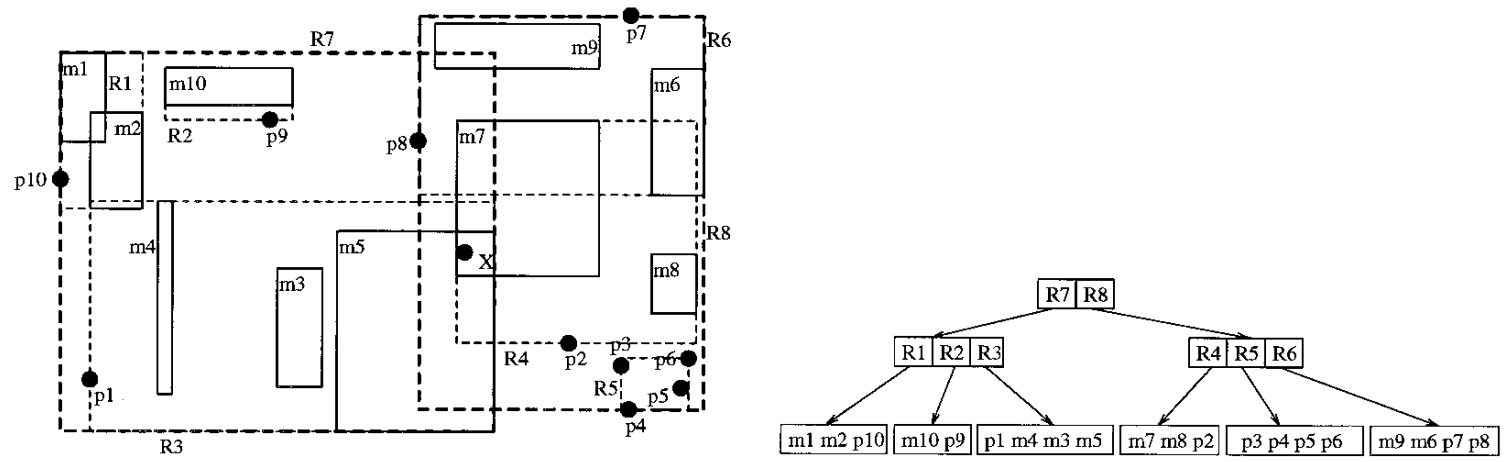

Figure 30. R-tree.

visit every index page. Figure 30 shows an R-tree for the running example. Remember that the mi denote the MBBs of the polygonal data objects ri. A point query with search point $\mathrm{X}$ results in two paths: $\mathrm{R} 8 \rightarrow \mathrm{R} 4 \rightarrow \mathrm{m} 7$ and R7 $\rightarrow \mathrm{R} 3 \rightarrow$ m5.

Because the R-tree only manages MBBs, it cannot solve a given search problem completely unless, of course, the actual data objects are intervalshaped. Otherwise the result of an Rtree query is a set of candidate objects, whose actual spatial extent has to be tested for intersection with the search space (cf. Fig. 2). This step, which may involve additional disk accesses and considerable computation, has not been taken into account in most published performance analyses [Guttman 1984; Greene 1989].

To insert an object 0 , we insert the minimum bounding interval $I^{\mathrm{d}}(0)$ and an object reference into the tree. In contrast to searching, we traverse only a single path from the root to the leaf. At each level we choose the child node $\nu$ whose corresponding interval $I^{\mathrm{d}}(\nu)$ needs the least enlargement to enclose the data object's interval $I^{\mathrm{d}}(\mathrm{o})$. If several intervals satisfy this criterion, Guttman proposes selecting the descendant associated with the smallest interval. As a result, we insert the object only once; that is, the object is not dispersed over several buckets. Once we have reached the leaf level, we try to insert the object. If this requires an enlargement of the corresponding bucket region, we adjust it appropriately and propagate the change upwards. If there is not enough space left in the leaf, we split it and distribute the entries among the old and the new page. Once again, we adjust each of the new intervals accordingly and propagate the split up the tree.

As for deletion, we first perform an exact match query for the object in question. If we find it in the tree, we delete it. If the deletion causes no underflow, we check whether the bounding interval can be reduced in size. If so, we perform this adjustment and propagate it upwards. If the deletion causes node occupation to drop below m, however, we copy the node content into a temporary node and remove it from the index. We then propagate the node removal up the tree, which typically results in the adjustment of several bounding intervals. Afterwards we reinsert all orphaned entries of the temporary node. Alternatively, we can merge the orphaned entries with sibling entries. In both cases, one may again have to adjust bounding intervals further up the tree.

In his original paper, Guttman [1984] discusses various policies to minimize the overlap during insertion. For node splitting, for example, Guttman suggests several algorithms, including a simpler one with linear time complexity and a more elaborate one with quadratic complexity. Later work by other 


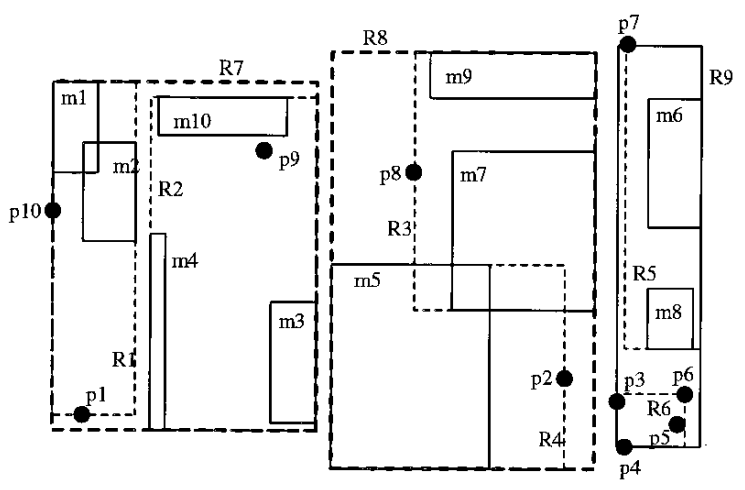

Figure 31. $\mathrm{R} *$-tree. researchers led to the development of more sophisticated policies. The packed R-tree [Roussopoulos and Leifker 1985], for example, computes an optimal partitioning of the universe and a corresponding minimal R-tree for a given scenario. However, it requires all data to be known a priori.

Other interesting variants of the $\mathrm{R}$ tree include the sphere tree by Oosterom [1990] and the Hilbert R-tree by Kamel and Faloutsos [1994]. The sphere tree corresponds to a hierarchy of nested d-dimensional spheres rather than intervals. The Hilbert R-tree combines the overlapping regions technique with space-filling curves (cf. Section 4.3). It first stores the Hilbert values of the data rectangles' centroids in a $\mathrm{B}^{+}$-tree, then enhances each interior $\mathrm{B}^{+}$-tree node by the MBB of the subtree below. This facilitates the insertion of new objects considerably. Together with a revised splitting policy, Kamel and Faloutsos report good performance results for both searches and updates. However, since their splitting policy takes only the objects' centroids into account, the performance of the structure is likely to deteriorate in the presence of large objects.

$\mathrm{Ng}$ and Kameda [1993] discuss how to support concurrency in R-trees by adopting the lock-coupling technique of B-trees [Bayer and Schkolnick 1977] to R-trees. Similarly, $\mathrm{Ng}$ and Kameda [1994] and Kornacker and Banks [1995] apply ideas of the B-link tree [Lehman and Yao 1981] to R-trees, yielding two structures both called the R-link tree. Kornacker and Banks empirically demonstrate that their R-link tree is superior to the R-tree using lock-coupling.

5.2.2 The $\mathrm{R} *$-Tree [Beckmann et al. 1990]. Based on a careful study of Rtree behavior under different data distributions, Beckmann et al. [1990] identified several weaknesses of the original algorithms. In particular, they confirmed the observation of Roussopoulos and Leifker [1985] that the insertion phase is critical for good search performance. The design of the $\mathrm{R}^{*}$-tree (see Figure 31) therefore introduces a policy called forced reinsert: If a node overflows, it is not split right away. Rather, $p$ entries are removed from the node and reinserted into the tree. The parameter $p$ may vary; Beckmann et al. suggest it should be about $30 \%$ of the maximal number of entries per page.

Another issue investigated by Beckmann et al. concerns the node-splitting policy. Although Guttman's R-tree algorithms tried only to minimize the area covered by the bucket regions, the $\mathrm{R}^{*}$ tree algorithms also take the following objectives into account.

-Overlap between bucket regions at the same tree level should be minimized. The less overlap, the smaller the probability that one has to follow multiple search paths.

-Region perimeters should be minimized. The preferred rectangle is the 
square, since this is the most compact rectangular representation.

-Storage utilization should be maximized.

The improved splitting algorithm of Beckmann et al. [1990] is based on the plane-sweep paradigm [Preparata and Shamos 1985]. In d dimensions, its time complexity is $\mathrm{O}(\mathrm{d} \cdot \mathrm{n} \cdot \log \mathrm{n})$ for a node with $\mathrm{n}$ intervals.

In summary, the $\mathrm{R}^{*}$-tree differs from the R-tree mainly in the insertion algorithm; del etion and searching are essentially unchanged. Beckmann et al. report performance improvements of up to $50 \%$ compared to the basic R-tree. Their implementation also shows that reinsertion may improve storage utilization. In broader comparisons, however, Hoel and Samet [1992] and Günther and Gaede [1997] found that the CPU time overhead of reinsertion can be substantial, especially for large page sizes; see Section 6 for further details.

One of the major insights of the $R^{*}$ tree is that node splitting is critical for the overall performance of the access method. Since a naive (exhaustive) approach has time complexity $\mathrm{O}\left(\mathrm{d} \cdot 2^{\mathrm{n}}\right)$ for $n$ given intervals, there is a need for efficient and optimal splitting policies. Becker et al. [1992] proposed a polynomial time algorithm that finds a balanced split, which also optimizes one of several possible objective functions (e.g., minimum sum of areas or minimum sum of perimeters). They assume in their analysis that the intervals are presorted in some specific order. More recently, Ang and Tan [1997] presented a new linear node splitting algorithm, based on a simple heuristic. According to the results reported, it outperforms its competitors.

Berchtold et al. [1996] proposed a modification of the R-tree called the $X$ tree that seems particularly well suited for indexing high-dimensional data. The X-tree reduces overlap among directory intervals by using a new organization: it postpones node splitting by introducing supernodes, that is, nodes larger than the usual block size. In order to find a suitable split, the $\mathrm{X}$-tree also maintains the history of previous splits.

5.2.3 The P-Tree [J agadish 1990c]. In many applications, intervals are not a good approximation of the data objects enclosed. In order to combine the flexibility of polygon-shaped containers with the simplicity of the R-tree, Jagadish [1990c] and Schiwietz [1993] independently proposed different variations of polyhedral trees or P-trees. To distinguish the two structures, we refer to the P-tree of J agadish [1990c] as the J P-tree and to the P-tree of Schiwietz [1993] as the SP-tree.

The J P-tree first introduces a variable number $\mathrm{m}$ of orientations in the $\mathrm{d}$-dimensional universe, where $\mathrm{m}>\mathrm{d}$. For instance, in two dimensions $(d=2)$ we may have four orientations $(m=4)$ : two parallel to the coordinate axes (i.e., iso-oriented) and two parallel to the two main diagonals. Objects are approximated by minimum bounding polytopes whose faces are parallel to these $m$ orientations. Clearly, the quality of the approximations is positively correlated with $m$. We can now map the original space into an m-dimensional orientation space, such that each (d-dimensional) approximating polytope $\mathrm{P}^{\mathrm{d}}$ turns into an m-dimensional interval $\mathrm{I}^{\mathrm{m}}$. Any point inside (outside) $\mathrm{P}^{\mathrm{d}}$ maps onto $\mathrm{a}$ point inside (outside) $\mathrm{I}^{\mathrm{m}}$, whereas the opposite is not necessarily true. To maintain the m-dimensional intervals, a large selection of SAMs is available; J agadish [1990c] suggests the R-tree or $\mathrm{R}^{+}$-tree (cf. Section 5.3.2) for this purpose.

An interesting feature of the J P-tree is the ability to add hyperplanes to the attribute space dynamically without having to reorganize the structure. By projecting the new intervals of the extended orientation space onto the old orientation space, it is still possible to use the old structure. Consequently, we can obtain an R-tree from a higherdimensional J P-tree structure by drop- 

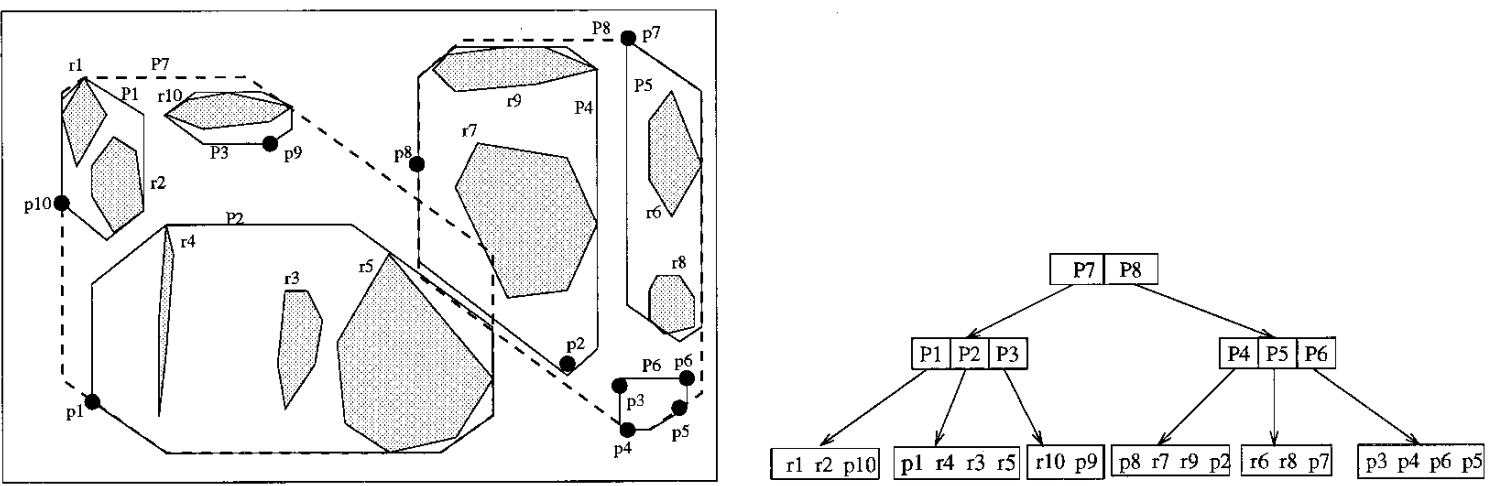

Figure 32. P-tree [J agadish 1990c].

ping all hyperplanes that are not isooriented.

The interior nodes of the J P-tree represent a hierarchy of nested polytopes, similar to the R-tree or the cell tree (cf. Section 5.3.3). Polytopes corresponding to different nodes at the same tree level may overlap. For search operations we first compute the minimum bounding polytope of the search region and map it onto an m-dimensional interval. The search efficiency then depends on the chosen PAM. The same applies for deletion.

The introduction of additional hyperplanes yields a better approximation, but it increases the size of the entries, thus reducing the fanout of the interior nodes. Experiments reported by J agadish [1990c] suggest that a 10-dimensional orientation space $(m=10)$ is a good choice for storing 2-dimensional lines $(d=2)$ with arbitrary orientation. This needs to be compared to a simple MBB approach. Although the latter technique may sometimes render poor approximations, the representation requires only four numbers per line. Storing a 10-dimensional interval, on the other hand, requires 20 numbers, that is, five times as many. Another drawback of the J P-tree is the fixed orientation of the hyperplanes. Figure 32 shows the running example for $\mathrm{m}=4$.

To overcome the problem of poor filtering, Brodsky et al. [1995] proposed methods for effectively computing a set of optimal axes for separating polyhe- dra. This work continues the line of work by J agadish [1990c] in the use of nonstandard axes for better filtering.

5.2.4 The P-Tree [Schi wietz 1993]. The P-Tree of Schiwietz, here called the SPtree, chooses a slightly different approach for storing polygonal objects that tries to combine the advantages of the cell tree and the $\mathrm{R}^{*}$-tree for the twodimensional case, while avoiding the drawbacks of both methods. Basically, the SP-tree is an R-tree whose interior nodes correspond to a nesting of polytopes rather than just rectangles. In general, the number of vertices (and ther efore the storage requirements) of a polytope are not bounded. Moreover, when used for approximating other objects, the accuracy of the approximation is positively correlated with the number of vertices of the approximating convex polygon. On the other hand, when used as index entries, there should be an upper bound in order to guarantee a minimum fanout of the interior nodes. To determine a reasonably good compromise between these conflicting objectives, extensive investigations have been conducted by Brinkhoff et al. [1993a] and Schiwietz [1993]. According to these studies, pentagons or hexagons seem to offer the best tradeoff between storage requirements and approximation quality.

If node splittings or insertions lead to additional vertices such that some bounding polygons have more vertices 

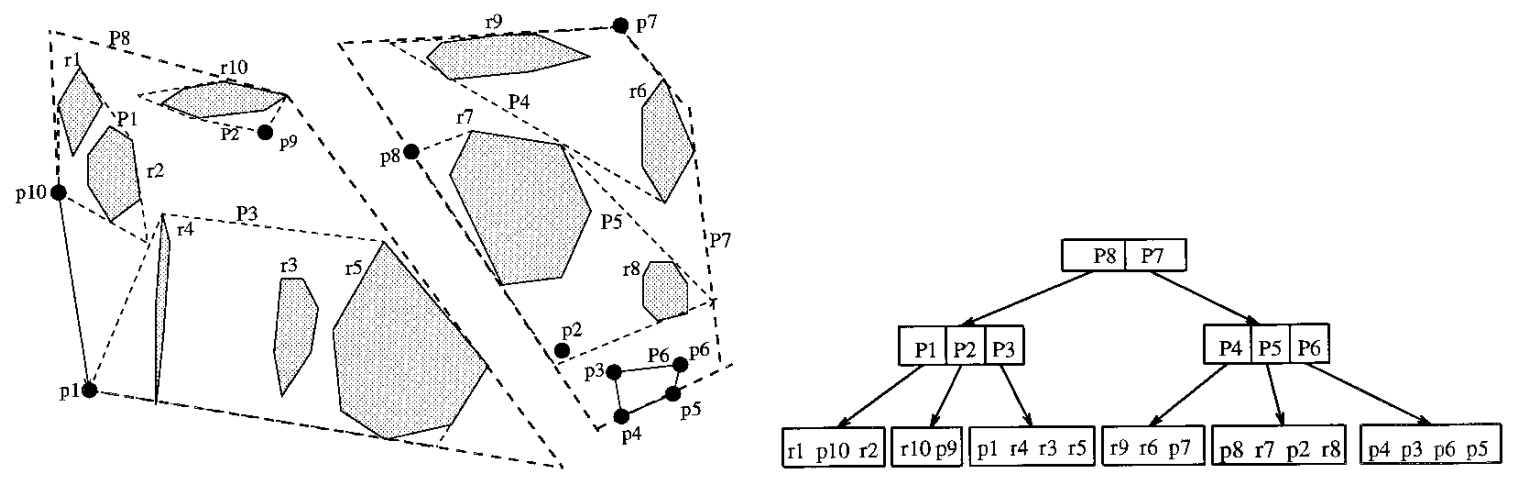

Figure 33. P-tree [Schiwietz 1993].

than the threshold, the surplus vertices are removed one by one. This leads to a larger area and therefore to a decrease of the quality of the approximation. To reduce overlap between the convex containers, Schiwietz suggests using a method similar to the $\mathrm{R}^{*}$-tree. Furthermore, in order to save storage space and to improve storage utilization, it is possible to restrict the number of orientations for the polygon edges (similar to the J P-tree).

Figure 33 shows the SP-tree for the running example. To our knowledge, no performance results have been reported so far for either of the two P-trees.

5.2.5 The SKD-Tree [Ooi et al. 1987; Ooi 1990]. A variant of the k-d-tree capable of storing spatially extended objects is the spatial k-d-tree or skd-tree. The skd-tree allows regions to overlap. To keep track of the mutual overlap, we store an upper and a lower bound with each discriminator, representing the maximal extent of the objects in the two subtrees. For example, consider the splitting hyperplane (discriminator) hxl depicted in Figure 34 and its upper and lower bounds bx1 and bx2, respectively. The solid lines are the splitting hyperplanes and the dashed lines represent the upper and lower bounds of the corresponding subtrees. $\mathrm{m} 3$ is the rectangle closest to hxl without crossing it, thus determining the maximum extent bx1 of the objects in the left (lower) subspace. Similarly, m5 determines the minimum extent bx2 for the right (up- per) subspace. If none of the objects placed in the corresponding subspace crosses the splitting hyperplane, the lower bound of the upper interval is greater than the discriminator, and the upper bound of the lower interval is less than $d_{k}$. Leaf nodes of the binary tree contain the minimal bounds (dotted lines) of the objects in the corresponding data page.

Prior to inserting an object $\mathrm{O}$, we determine its centroid and its MBB. By comparing the centroid with the stored discriminators, we determine the next child to be inspected. Note that there is no ambiguity. During insertion, we have to adjust the upper and lower bounds for extended objects accordingly. U pon reaching the data node level, we test whether there is enough space available to accommodate the object. If so, we insert the object; otherwise we split the data node and insert the new discriminator into the skd-tree. Likewise, the bounds of the new subspaces need to be adjusted.

As usual, searching starts at the root and corresponds to a top-down tree traversal. At each interior node we check the discriminator and the boundaries to decide which child(ren) to visit next.

Deleting an object starts with an exact match query to determine the correct leaf node. If a deletion causes an underflow, we insert the remaining entries into the sibling data node and remove the splitting hyperplane. If this insertion results in an overflow, we split 


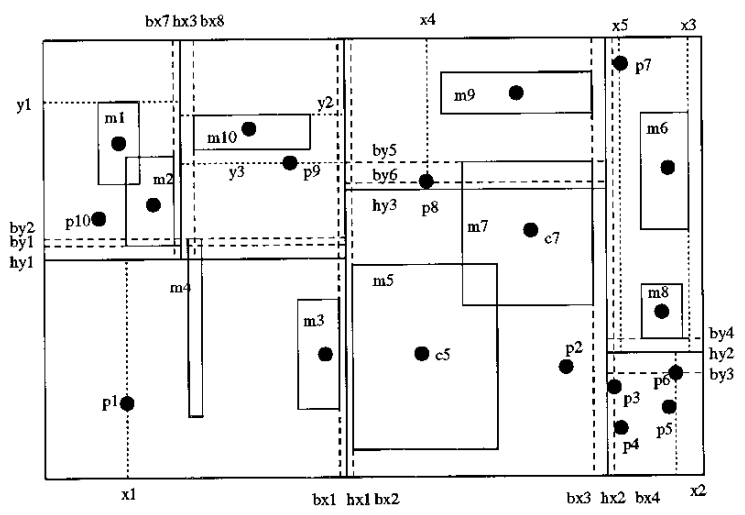

Figure 34. SKD-tree. the page and insert the new hyperplane into the skd-tree. If no merge with a sibling leaf node is possible, we delete that leaf and its parent node. By redirecting the reference of the latter to its sibling (interior) node, we extend the subspace of the sibling. All affected entries are reinserted.

According to the results reported in Ooi [1990] and Ooi et al. [1991], the skd-tree is competitive with the R-tree both in storage utilization and search efficiency.

5.2.6 The GBD-Tree [Ohsawa and Sakauchi 1990]. The GBD-tree (generalized BD-tree) is an extension of the BD-tree [Ohsawa and Sakauchi 1983] that allows for secondary storage management and supports the management of extended objects. The BD-tree is a binary tree, but the GBD-tree is a balanced multiway tree that stores spatial objects as a hierarchy of minimum bounding boxes. Each leaf node (bucket) stores the MBBs of those objects whose centroids are contained in the corresponding bucket region. Each interior node stores the MBB of the (usually overlapping) MBBs of its descendants. The intervals are encoded using the same DZ-expressions as described in Section 3.2.3.

The one advantage of the GBD-tree over the R-tree is that insertions and deletions may be processed more efficiently, due to the encoding scheme and the placement by centroid. The latter

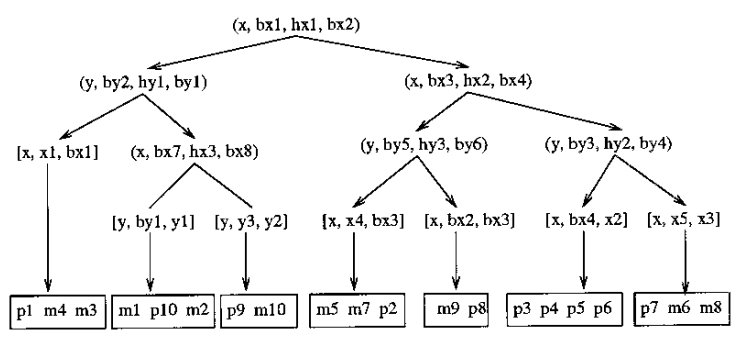

point enables the GBD-tree to perform an insertion along a single path from the root to a leaf. However, no apparent advantage is gained in search performance. The reported performance experiments [Ohsawa and Sakauchi 1990] compare only storage utilization and insertion performance with the R-tree. The most important comparison, that of search performance, is omitted.

Figure 35 depicts a GBD-tree for the running example. The partitioning on the left-hand side shows the minimum bounding boxes (dotted or dashed) and the underlying intervals (Peano regions).

Among the approaches similar to the GBD-tree are an extension of the buddy tree by Seeger [1991] and the extension of the BANG file to handle extended spatial objects [F reeston 1989b].

5.2.7 PLOP-Hashing [Kriegel and Seeger 1988; Seeger and Kriegel 1988]. Piecewise linear order-preserving (PLOP) hashing [Seeger and Kriegel 1988] is a variant of hashing that allows the storage of extended objects without transforming them into points. An earlier version of this structure [Kriegel and Seeger 1988] was only able to handle multidimensional point data.

PLOP-hashing partitions the universe in a similar way to the grid file: extended objects may span more than one directory cell. Hyperplanes extend al ong the axes of the data space. F or the organization of these hyperplanes, PLOP- 


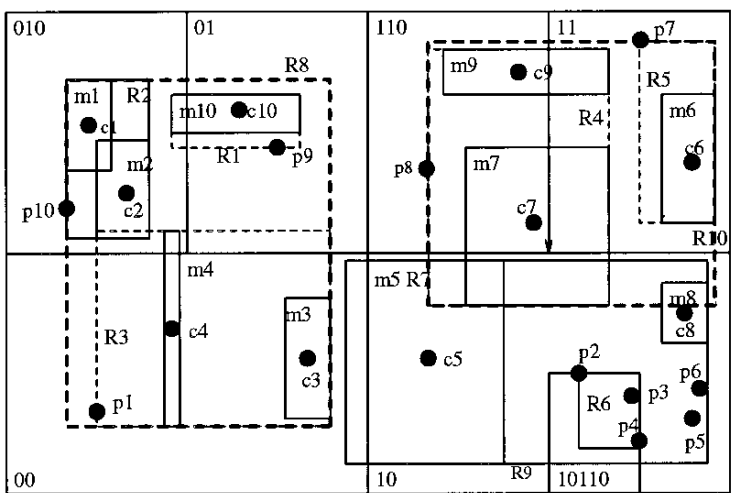

Figure 35.

hashing uses $d$ binary trees, where $d$ is the dimension of the universe. Each interior node of such a binary tree corresponds to a $(d-1)$-dimensional isooriented hyperplane. The leaf nodes represent d-dimensional subspaces forming slices of the universe.

Figure 36 depicts the binary trees for both axes together with the slices formed by them. By using the index entries that are stored in the leaf nodes, we can easily identify the data page for which we are looking. To do this efficiently, we have to keep the d binary trees in main memory, similar to the scales of the grid file. For further speedup, the leaf nodes of each binary tree are linked to each other. In Figure 36 this is suggested by the arrows attached to the leaves of the trees. To handle extended objects, we enlarge the storage representation of each slice by a lower and an upper bound. These bounds indicate the minimum and the maximum extension along the current dimension of all objects stored in the slice at hand.

Insertion is straightforward and similar to the grid file. To avoid ambiguities, PLOP-hashing uses the centroid of the object to determine the data bucket in which to place the object. In the case of node splitting and deletion we have to adjust the respective upper and lower bounds. It should be further noted that PLOP-hashing can be easily modified so that it supports clipping rather than overlapping regions.

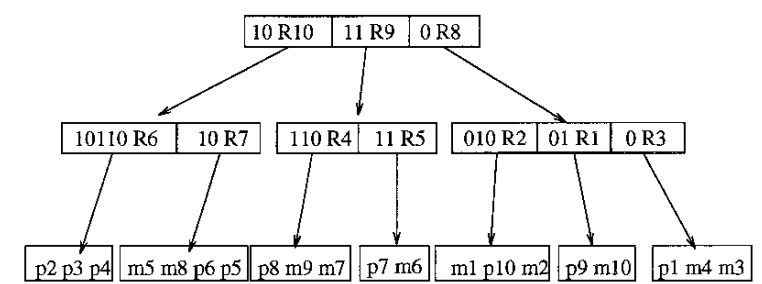

GBD-tree.

Analytical experiments indicate that PLOP-hashing is superior to the R-tree and $\mathrm{R}^{+}$-tree for uniform data distributions [Seeger and Kriegel 1988].

\subsection{Clipping}

Clipping-based schemes do not allow any overlaps between bucket regions; they have to be mutually disjoint. A typical access method of this kind is the $\mathrm{R}^{+}$-tree [Stonebraker et al. 1986; Sellis et al. 1987], a variant of the R-tree that allows no overlap between regions corresponding to nodes at the same tree level. As a result, point queries follow a single path starting at the root, which means efficient searches.

The main problems with clippingbased approaches relate to the insertion and deletion of data objects. During insertion, any data object that spans more than one bucket region has to be subdivided along the partitioning hyperplanes. Eventually, several bucket entries have to be created for the same object. Each bucket stores either the geometric description of the complete object (object duplication) or the geometric description of the corresponding fragment with an object reference. In any case, data about the object are dispersed among several data pages (spanning property). The resulting redundancy [Orenstein 1989a,b; Günther and Gaede 1997] may cause not only an increase in the average search time, but 

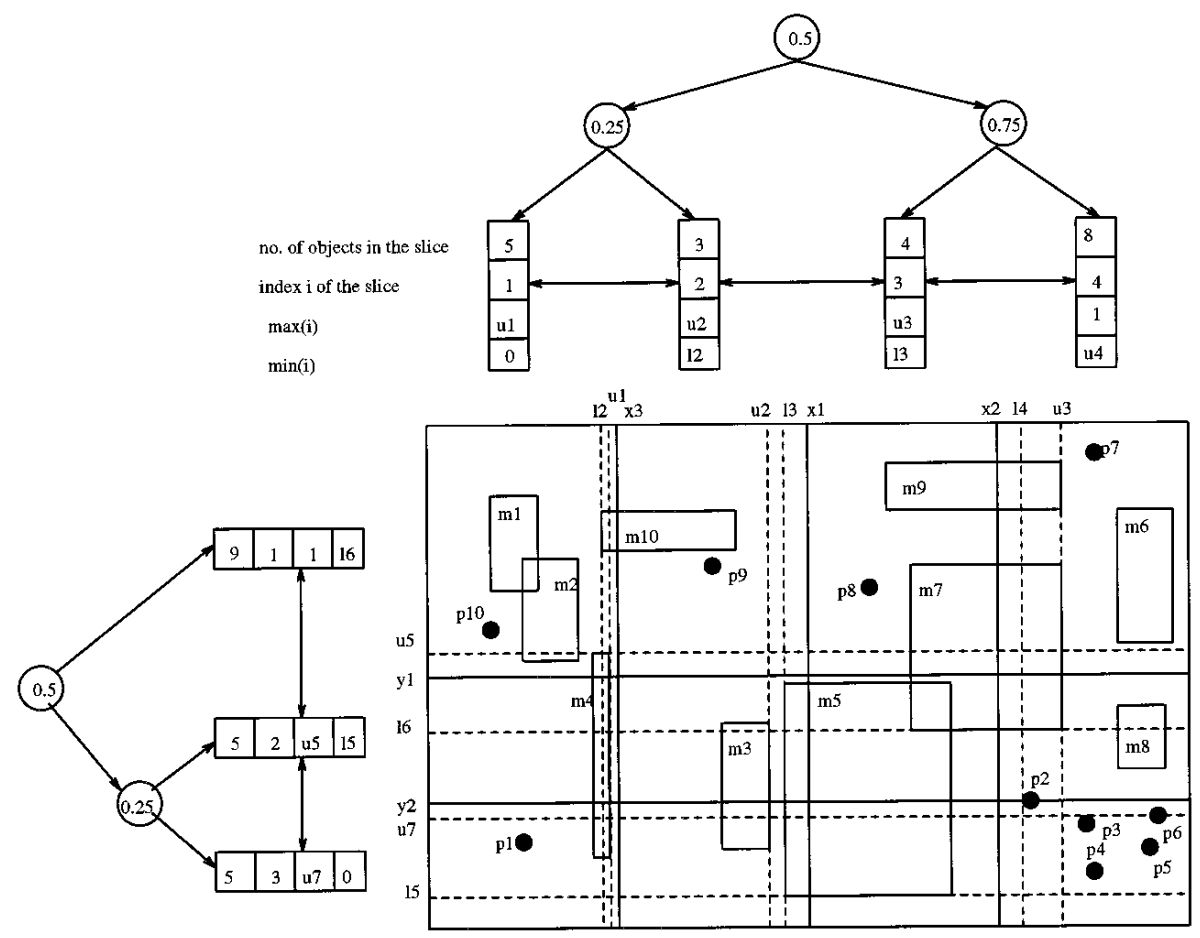

Figure 36. PLOP-hashing.

also an increase in the frequency of bucket overflows.

A second problem applies to clippingbased access methods that do not partition the complete data space. In that case, the insertion of a new data object may lead to the enlargement of several bucket regions. Whenever the object (or a fragment thereof) is passed down to a bucket (or, in the case of a tree structure, an interior node) whose region does not cover it, the region has to be extended. In some cases, such an enlargement is not possible without getting an overlap with other bucket regions; this is sometimes called the deadlock problem of clipping. Because overlap is not allowed, one has to compute a new region structure, which can become very complicated. It may in particular cause further bucket overflows and insertions, which can lead to a chain reaction and, in the worst case, a complete breakdown of the structure [Günther and Bilmes 1991]. Access methods partitioning the complete data space do not suffer from this problem.
A final problem concerns the splitting of buckets. There may be situations where a bucket (and its corresponding region) has to be split but there is no splitting hyperplane that splits none (or only a few) of the objects in that bucket. The split may then trigger other splits, which may become problematic with increasing size of the database. The more objects are inserted, the higher the probability of splits and the smaller the average size of the bucket regions. New objects are therefore split into a larger number of smaller fragments, which may in the worst case once again lead to a chain reaction. To alleviate these problems, Günther and Noltemeier [1991] suggest storing large objects (which are more likely to be split into a large number of fragments) in special buckets called oversize shelves, instead of inserting them into the structure.

5.3.1 The Extended k-d-Tree [Matsuyama et al. 1984]. One of the earliest extensions of the adaptive k-d-tree that could handle extended objects was the 

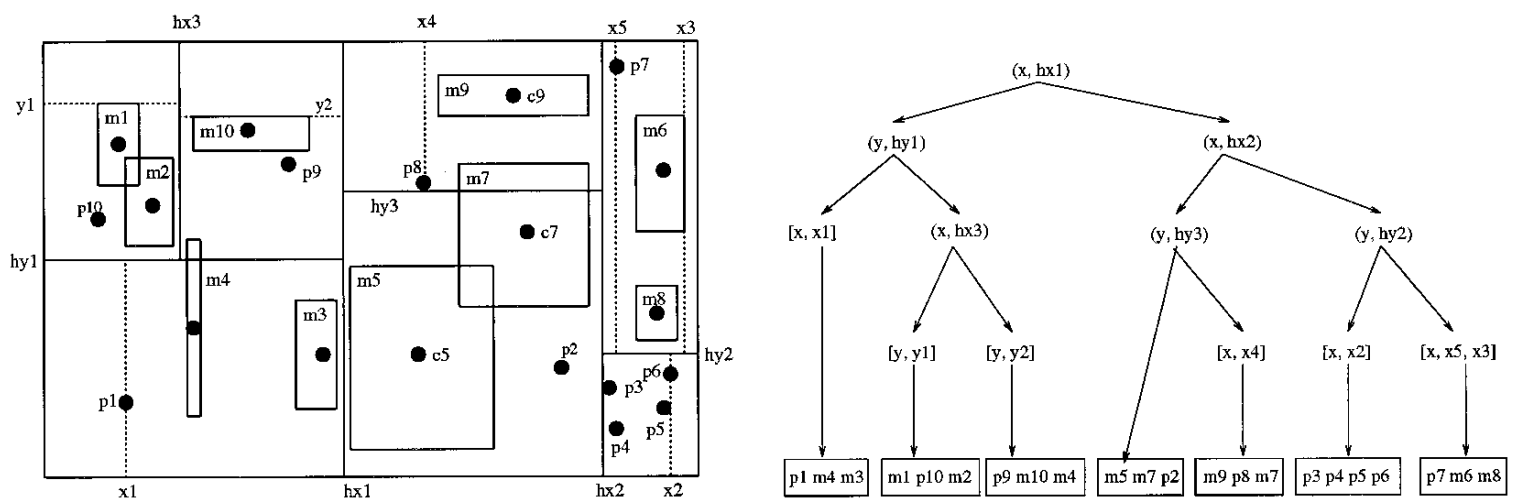

Figure 37. Extended k-d-tree.

extended k-d-tree. In contrast to the skd-tree (Section 5.2.5), the extended $\mathrm{k}$-d-tree is based on clipping. Each interior tree node corresponds to a $(d-1)$ dimensional partitioning hyperplane, represented by the dimension (e.g., $x$ or y) and the splitting coordinate (the discriminator). A leaf node corresponds to a rectangular subspace and contains the address of the data page describing that subspace. Data pages may be referenced by multiple leaf nodes.

To insert an object, we start at the root of the k-d-tree. At each interior node, we test for intersection with the stored hyperplane. Depending on the location of the object relative to the hyperplane, we either move on to the corresponding child node, or we clip the object by the hyperplane and follow both branches. This procedure guarantees that we insert the object in all overlapping bucket regions. If a data page cannot accommodate the additional object, we split the page by a new hyperplane. The splitting dimension is perpendicular to the dimension with the greatest extension. After distributing the entries of the data page among the two new pages, we insert the hyperplane into the k-d-tree. Note that this may in turn cause some objects to be split, which may lead to further page overflows. To delete an object, we have to visit all subspaces intersecting the object and delete the stored object identifier. If a data page is empty due to deletion, we remove it and mark all leaf nodes pointing to that page as NIL. No merging of sibling nodes is performed.

Figure 37 depicts an extended k-dtree for the running example. Rectangle $\mathrm{m} 7$ has been clipped and inserted into two nodes. Most partitions contain one or two additional bounding hyperplanes (dotted lines) to provide a better localization of the objects in the corresponding subspace.

5.3.2 The $\mathrm{R}^{+}$-Tree [Stonebraker et al. 1986; Sellis et al. 1987]. To overcome the problems associated with overlapping regions in the R-tree, Sellis et al. [1987] introduced an access method called the $\mathrm{R}^{+}$-tree. Unlike the R-tree, the $\mathrm{R}^{+}$-tree uses clipping; that is, there is no overlap between index intervals I $^{\mathrm{d}}$ at the same tree level. Objects that intersect more than one index interval have to be stored on several different pages. As a result of this policy, point searches in $\mathrm{R}^{+}$-trees correspond to single-path tree traversals from the root to one of the leaves. They therefore tend to be faster than the corresponding R-tree operation. Range searches usually lead to the traversal of multiple paths in both structures.

When inserting a new object 0 , we may have to follow multiple paths, depending on the number of intersections of the MBB $\mathrm{I}^{\mathrm{d}}(\mathrm{o})$ with index intervals. During the tree traversal, $I^{d}(0)$ may be split into $\mathrm{n}$ disjoint fragments $\mathrm{I}_{\mathrm{i}}^{\mathrm{d}}(\mathrm{o})$ $\left(\cup_{i=1}^{n} I_{i}^{d}(0)=I^{d}(0)\right)$. Each fragment is then placed in a different leaf node $\nu_{\mathrm{i}}$. 

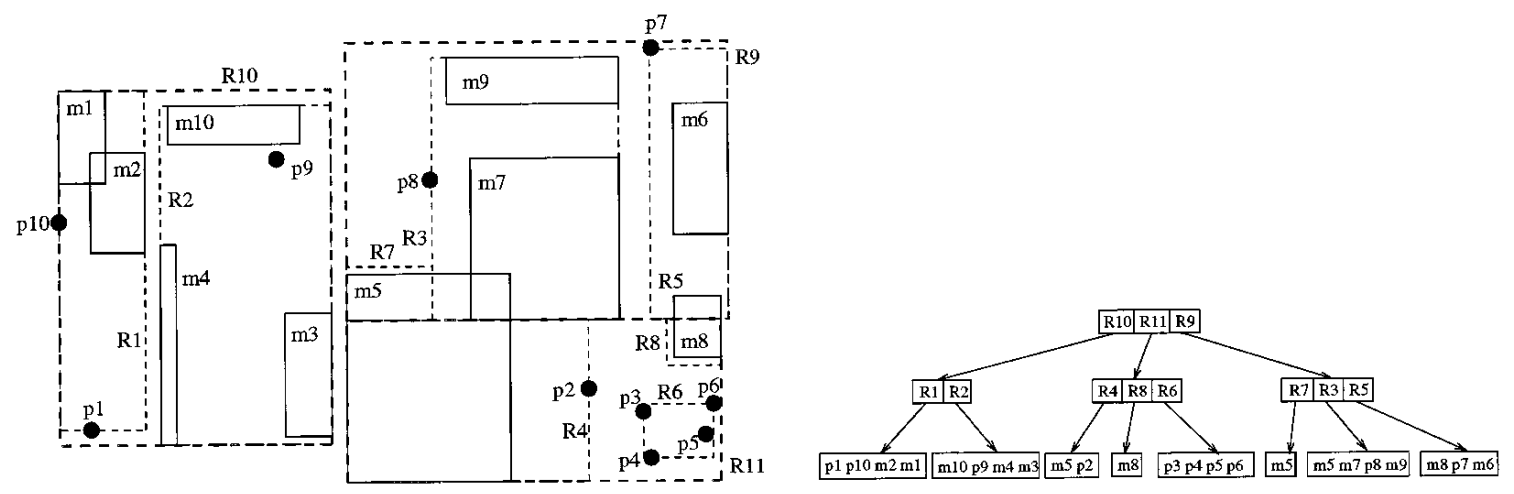

Figure 38. $\mathrm{R}^{+}$-tree.

Provided that there is enough space, the insertion is straightforward. If the bounding interval $\mathrm{I}^{\mathrm{d}}(\mathrm{o})$ overlaps space that has not yet been covered, we have to enlarge the intervals corresponding to one or more leaf nodes. Each of these enlargements may require considerable effort because overlaps must be avoided. In some rare cases, it may not be possible to increase the current intervals in such a way that they cover the new object without some mutual overlap [Günther 1988; Ooi 1990]. In case of such a deadlock, some data intervals have to be split and reinserted into the tree.

If a leaf node overflows it has to be split. Node splittings work similarly to the case of the R-tree. An important difference, however, is that splits may propagate not only up the tree, but also down the tree. The resulting forced split of the nodes below may lead to several complications, including further fragmentation of the data intervals; see, for example, the rectangles $\mathrm{m} 5$ and $\mathrm{m} 8$ in Figure 38.

For deletion, we first locate all the data nodes where fragments of the object are stored and remove them. If storage utilization drops below a given threshold, we try to merge the affected node with its siblings or to reorganize the tree. This is not always possible, which is the reason why the $\mathrm{R}^{+}$-tree cannot guarantee a minimum space utilization.
5.3.3 The Cell Tree [Günther 1988]. The main goal in the design of the cell tree [Günther 1988; 1989] was to facilitate searches on data objects of arbitrary shapes, that is, especially on data objects that are not intervals themselves. The cell tree uses clipping to manage large spatial databases that may contain polygons or higher-dimensional polyhedra. It corresponds to a decomposition of the universe into disjoint convex subspaces. The interior nodes correspond to a hierarchy of nested polytopes and each leaf node corresponds to one of the subspaces (Figure 39). Each tree node is stored on one disk page.

To avoid some of the disadvantages resulting from clipping, the convex polyhedra are restricted to be subspaces of a BSP (binary space partitioning). Therefore we can view the cell tree as a combination of a BSP- and an $\mathrm{R}^{+}$-tree, or as a BSP-tree mapped on paged secondary memory. In order to minimize the number of disk accesses that occur during a search operation, the leaf nodes of a cell tree contain all the information required for answering a given search query; we load no pages other than those containing relevant data. This is an important advantage of the cell tree over the R-tree and related structures.

Before inserting a nonconvex object, we decompose it into a number of convex components whose union is the orig- 


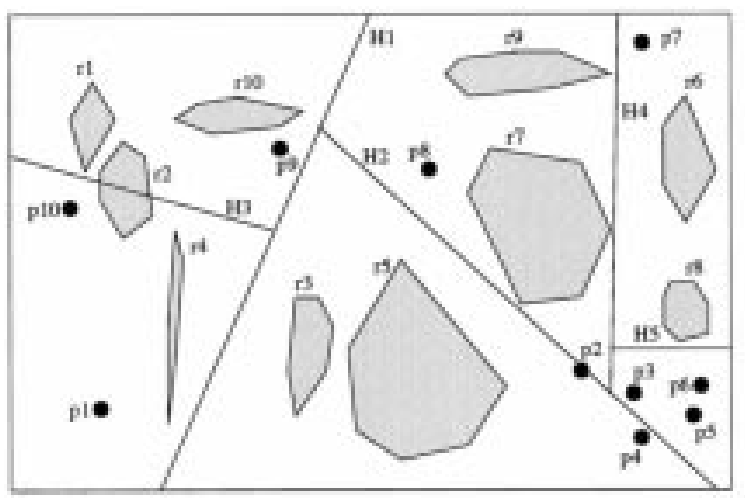

Figure 39. Cell tree. inal object. The components do not have to be mutually disjoint. All components are assigned the same object identifier and inserted into the cell tree one by one. Due to clipping, we may have to subdivide each component into several cells during insertion, because it overlaps more than one subspace. Each cell is stored in one leaf node of the cell tree. If an insertion causes a disk page to overflow, we have to split the corresponding subspace and cell tree node and distribute its descendants between the two resulting nodes. Each split may propagate up the tree.

For point searches, we start at the root of the tree. Using the underlying BSP partitioning, we identify the subspace that includes the search point and continue the search in the corresponding subtree. This step is repeated recursively until we reach a leaf node, where we examine all cells to see whether they contain the search point. The solution consists of those objects that contain at least one of the cells that qualify. A similar algorithm exists for range searches. A performance evaluation of the cell tree [Günther and Bilmes 1991] shows that it is competitive with other popular spatial access methods.

Figure 39 shows our running example with five partitioning hyperplanes, each of them stored in the interior nodes. Even though the partitioning by means of the BSP-tree offers more flexibility than rectilinear hyperplanes, clipping

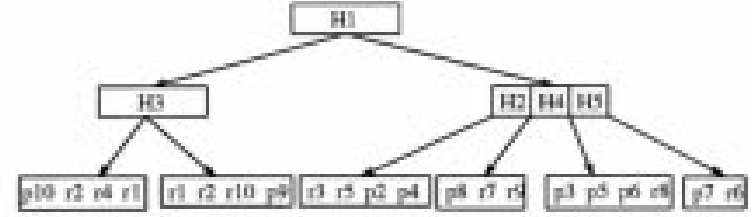

objects may be inevitable. In Figure 39, we had to split $r 2$ and insert the resulting cells into two pages.

As do all structures based on clipping, the cell tree has to cope with the fragmentation of space, which becomes increasingly problematic as more objects are inserted into the tree. After some time, most new objects will be split into fragments during insertion. To avoid the negative effects of this fragmentation, Günther and Noltemeier [1991] proposed the concept of oversize shelves. Oversize shelves are special disk pages attached to the interior nodes of the tree that accommodate objects which would have been split into too many fragments if they had been inserted regularly. The authors propose a dynamically adjusting threshold for choosing between placing a new object on an oversize shelf or inserting it regularly. Performance results of Günther and Gaede [1997] show substantial improvements compared to the cell tree without oversize shelves.

\subsection{Multiple Layers}

The multiple layer technique can be regarded as a variant of the overlapping regions approach, because data regions of different layers may overlap. However, there are several important differences. First, the layers are organized in a hierarchy. Second, each layer partitions the complete universe in a different way. Third, data regions within a 
layer are disjoint; that is, they do not overlap. Fourth, the data regions do not adapt to the spatial extensions of the corresponding data objects.

In order to get a better understanding of the multilayer technique, we discuss how to insert an extended object. First, we try to find the lowest layer in the hierarchy whose hyperplanes do not split the new object. If there is such a layer, we insert the object into the corresponding data page. If the insertion causes no page to overflow, we are done. Otherwise, we must split the data region by introducing a new hyperplane and distribute the entries accordingly. Objects intersecting the hyperplane have to be moved to a higher layer or an overflow page. As the database becomes populated, the data space of the lower layers becomes more and more fragmented. As a result, large objects keep accumulating on higher layers of the hierarchy or, even worse, it is no longer possible to insert objects without intersecting existing hyperplanes.

The multilayer approach seems to offer one advantage over the overlapping regions technique: a possibly higher selectivity during searching due to the restricted overlap of the different layers. However, there are al so several disadvantages: the multilayer approach suffers from fragmentation, which may render the technique inefficient for some data distributions; certain queries require the inspection of all existing layers; it is not clear how to cluster objects that are spatially close to each other but in different layers; and there is some ambiguity about the layer in which to place the object.

An early static multilayer access method is the MX-CIF quadtree [Kedem 1982; Abel and Smith 1983; Samet 1990b]. This structure stores each extended spatial object with the quadtree node whose associated quadrant provides the tightest fit without intersecting the object. Objects within a node are organized by means of binary trees.

Sevcik and Koudas [1996] later proposed a similar SAM based on multiple layers, called the filter tree. As in the $\mathrm{MX}-\mathrm{CIF}$ quadtree, each layer is the result of a regular subdivision of the universe. A new object is assigned to a unique layer, depending on the object's position and extension. Objects within one layer are first sorted by the Hilbert code of their center, then packed into data pages of a given size. Finally, the largest Hilbert code of each data page, together with its reference, is inserted into a B-tree.

We continue with a detailed description of two dynamic SAMs based on multiple layers.

5.4.1 The Multilayer Grid File [Six and Widmayer 1988]. Yet another variant of the grid file capable of handling extended objects is the multilayer grid file (not to be confused with the multilevel grid file of Whang and Krishnamurthy [1985]). The multilayer grid file consists of an ordered sequence of grid layers. Each of these layers corresponds to a separate grid file (with freely positionable splitting hyperplanes) that covers the whole universe. A new object is inserted into the first grid file in the sequence that does not imply any clipping of the object. This is an important difference from the twin grid file (see Section 4.1.4), where objects can be moved freely between the two layers. If one of the grid files is extended by adding another splitting hyperplane, those objects that would be split have to be moved to another layer. Figure 40 illustrates a multilayer grid file with two layers for the running example.

In the multilayer grid file, the size of the bucket regions typically increases within the sequence; that is, larger objects are more likely to find their final location in later layers. If a new object cannot be stored in any of the current layers without clipping, a new layer has to be allocated. An alternative is to allow clipping only for the last layer. Six and Widmayer claim that $\mathrm{d}+1$ layers are sufficient to store a set of d-dimen- 


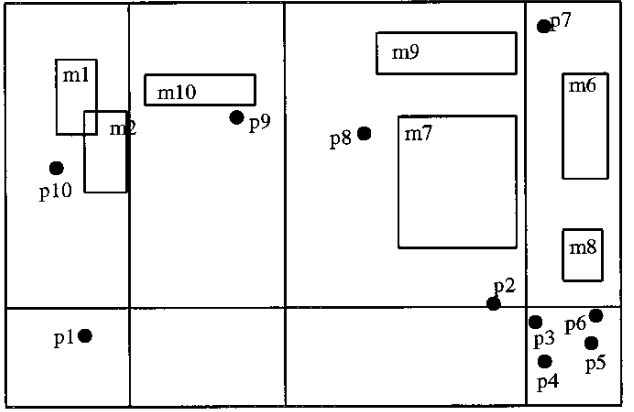

1st layer

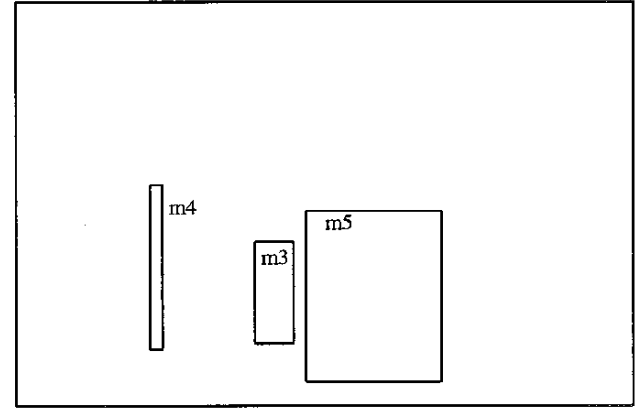

2nd layer

Figure 40. Multilayer grid file.

sional intervals without clipping if the hyperplanes are cleverly chosen.

For an exact match query, we can easily determine from the scales which grid file in the sequence is supposed to hold the search interval. Other search queries, in particular point and range queries, are answered by traversing the sequence of layers and performing a corresponding search on each grid file. The performance results reported by Six and Widmayer [1988] suggest that the multilayer grid file is superior to the conventional grid file, using clipping to handle extended objects. Possible disadvantages of the multilayer grid file include low storage utilization and expensive directory maintenance.

5.4.2 The R-File [Hutflesz et al. 1990]. To overcome some of the problems of the multilayer grid file, Hutflesz et al. [1990] proposed an alternative structure for managing sets of rectangles called the R-file; see Figure 41 for an example. In order to avoid the low storage utilization of the multilayer grid file, the R-file uses a single directory. The universe is partitioned similarly to the BANG file: splitting hyperplanes cut the universe recursively into equal parts, and z-ordering is used to encode the resulting bucket regions. In contrast to the BANG file, however, there are no excisions. Bucket regions may overlap, and there is no clipping. Each data interval is stored in the bucket with the smallest region that contains it entirely; overflow pages may be necessary in some cases.

An interesting feature of the R-file is its splitting algorithm. Rather than cutting a bucket region into two halves, we retain the original bucket region and create a new bucket for one of the two halves of that original region. Data intervals are then assigned to the new bucket if and only if they are completely contained in the corresponding region. The half is chosen in such a way that the distribution of data intervals between the two resulting buckets is most even. Once a region has been split, it may subsequently be split again, using the same algorithm. Since objects that are located near the middle of the universe are likely to intersect the partitioning hyperplanes, they are often assigned to the cell region corresponding to the whole universe. Thus objects in that cell tend to cluster near the splitting hyperplanes (cf. rectangle r 5 in Figure 41).

To avoid searching dead space, the R-file maintains minimum enclosing boxes of the stored objects, called search regions. As shown by Hutflesz et al. [1990], this feature, together with the z-encoding of the partitions, make the R-file competitive to the R-tree. One drawback of the R-file is the fact that it partitions the entire space, whereas the R-tree indexes only the part of the universe that contains objects. For data distributions that are nonuniform, the 

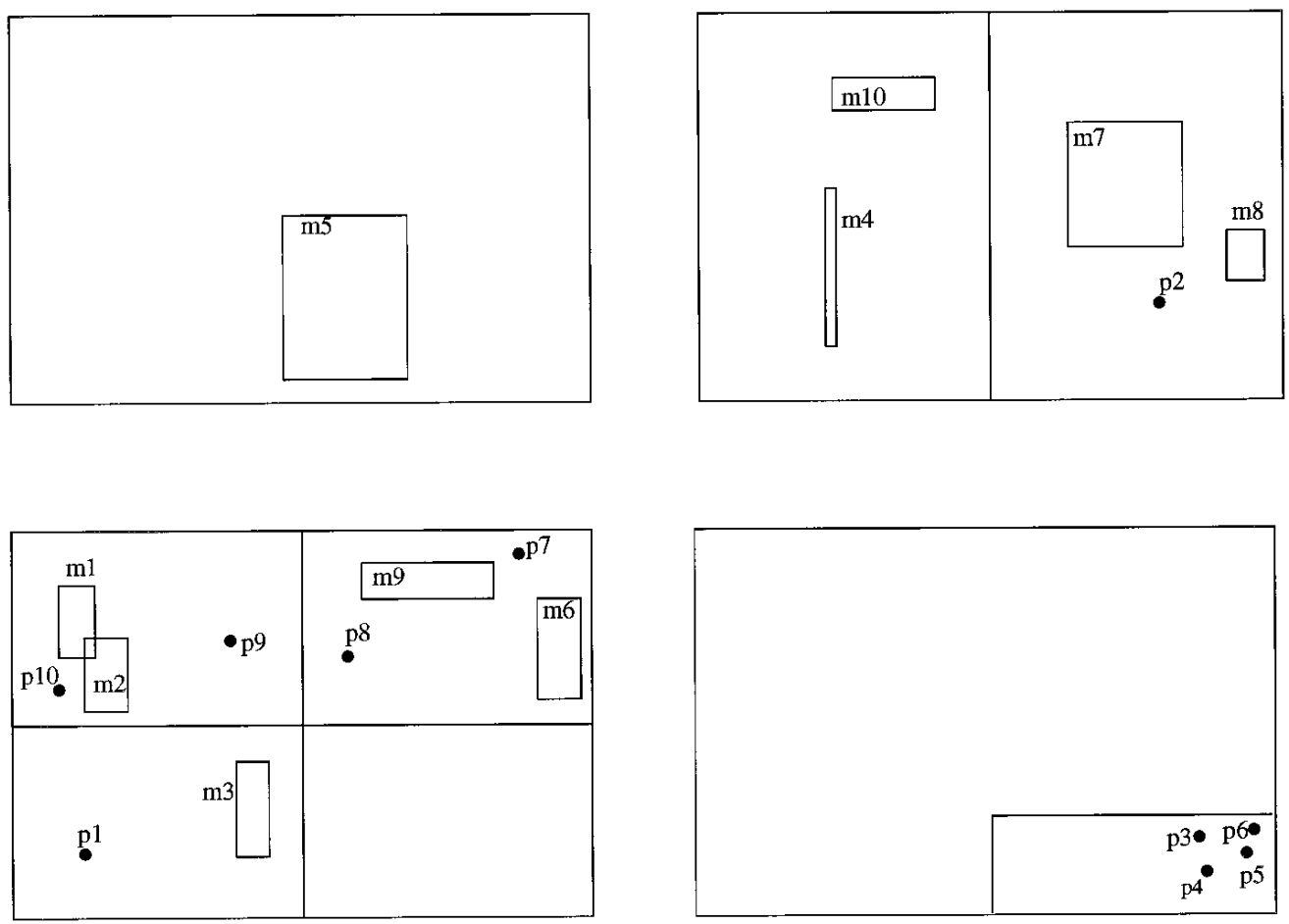

Figure 41. R-file.

R-file therefore often performs poorly. This disadvantage is something that the R-file shares with the grid file. Widmayer [1991] also notes that the R-file is "al gorithmically complicated."

\section{COMPARATIVE STUDIES}

In this section, we give a brief overview of theoretical and experimental results on the comparison of different access methods. Unfortunately, the number of such evaluations, especially theoretical analyses, is rather limited.

Greene [1989] compares the search performance of the R-tree, the k-d-Btree, and the $\mathrm{R}^{+}$-tree for 10,000 uniformly distributed rectangles of varying size. Query parameters include the size of the query rectangles and the page size. Greene's study shows that the k-dB-tree can never really compete with the two R-tree variants. On the other hand, there is not much difference between the $\mathrm{R}^{+}$-tree and the R-tree, even though the former is significantly more difficult to code. As expected, the $\mathrm{R}^{+}$- tree performs better when there is less overlap between the data rectangles.

Kriegel et al. [1990] present an extensive experimental study of accessmethod performance for a variety of point distributions. The study involves four point access methods: the hB-tree, the BANG file, the two-level grid file, and the buddy tree. The authors decided not to include PLOP-hashing since its performance suffers considerably for nonuniform data. The $\mathrm{zkdB}^{+}$-tree of Orenstein and Merrett [1984] was also not included since the authors considered both the BANG file and the hBtree as improvements of that strategy. Finally, Kriegel et al. did not include quantile hashing although they claim [Kriegel and Seeger 1987, 1989] that this structure is very efficient for nonuniform data.

According to the benchmarks, the buddy tree and, to some degree, the BANG file outperform all other structures. The reported results show in an impressive way how the performance of 
the access methods studied varies with different data distributions and query range sizes. For clustered data and a query range of size $10 \%$ of the size of the universe, there is almost no performance difference between the buddy tree and the BANG file. If the size of the query range drops to only $0.1 \%$ of the size of the universe; however, the buddy tree performs about twice as fast.

For extended objects, Kriegel et al. [1990] compared the R-tree and PLOPhashing with the buddy tree and the BANG file. The latter two techniques were enhanced by the transformation technique to handle rectangles. Once again, the buddy tree and the BANG file outperformed the other two access methods for nearly all data distributions. Note that the benchmarks measured only the number of page accesses but not the CPU time.

Beckmann et al. [1990] compared the $\mathrm{R}$ *-tree with several variants of the $\mathrm{R}$ tree for a variety of data distributions. Besides the performance of the different structures for point, intersection, and enclosure queries for varying query region sizes, they also compared spatial join performance. The $\mathrm{R}^{*}$-tree is the clear winner for all data distributions and queries, and it also has the best storage utilization and insertion times. A comparison for point data confirms these results. Similarly to previous performance measurements, only the number of disk accesses is measured. A related study by Kamel and Faloutsos [1994] finds even better search results for the Hilbert R-tree, whereas updates take about the same time as for the $\mathrm{R}^{*}$-tree. The impact of global clustering on the search performance of the $\mathrm{R} *$ tree was investigated by Brinkhoff and Kriegel [1994]. Kamel et al. [1996] use Hilbert codes for bulk insertion into dynamic $\mathrm{R} *$-trees.

Seeger [1991] studied the relative performance of clipping, overlapping regions, and transformation techniques implemented on top of the buddy tree. $\mathrm{He}$ also included the two-level grid file and the $\mathrm{R}^{*}$-tree in the comparison. The buddy tree with clipping and the grid file failed completely for certain distributions, since they produced unmanageably large files. The transformation technique supports fast insertions at the expense of low storage utilization. The $\mathrm{R}^{*}$-tree, on the other hand, requires fairly long insertion times but offers good storage utilization. For intersection and containment queries, the buddy tree combined with overlapping regions is continuously superior to the buddy tree with transformation. The performance advantage of the overlapping regions technique decreases for larger query regions, even though the buddy tree with transformation never outperforms the buddy tree with overlapping regions. When the data set contains uniformly distributed rectangles of varying size, the buddy tree with clipping outperforms the other techniques for intersection and enclosure queries. For some queries the buddy tree with overlapping performs slightly better than the $\mathrm{R}^{*}$-tree.

Ooi [1990] compares a static and a dynamic variant of the skd-tree with the packed R-tree described by Roussopoulos and Leifker [1985]. For large page sizes, the skd-tree clearly outperforms the R-tree in terms of page accesses per search operation. The space requirements of the skd-tree, however, are higher than those of the R-tree. Since the skd-tree stores the extended objects by their centroids, containment queries are answered more efficiently than by the R-tree. This behavior is clearly reflected in the performance results. A comparison with the extended k-d-tree, enhanced by overflow pages, suggests that the skd-tree is superior, although the extended k-d-tree (which is based on clipping) performs rather well for uniformly distributed data.

Günther and Bilmes [1991] compare the R-tree to two clipping-based access methods, the cell tree and the $\mathrm{R}^{+}$-tree. Unlike most studies, the data sets consist of convex polygons instead of just rectangles. The cell tree requires up to twice as much space as its competitors. 
However, the average number of page accesses per search operation is less than for the other two access methods. Moreover, this advantage tends to increase with the size of the database and the size of the query regions. Besides measurements on the number of page faults, CPU time measurements are also given.

Günther and Gaede [1997] compare the original cell tree as presented by Günther [1989] with the cell tree with oversize shelves [Günther and Noltemeier 1991], the $\mathrm{R}^{*}$-tree [Beckmann et al. 1990], and the hB-tree [Lomet and Salzberg 1989] for some real cartographic data. There is a slight performance advantage of the cell tree with oversize shelves compared to the $\mathrm{R}^{*}$ tree and the hB-tree, but a major difference from the original cell tree. An earlier comparison using artificially generated data can be found in Günther [1991]. Both studies suggest that oversize shelves may lead to significant improvements for access methods with clipping.

Oosterom [1990] compares the query times of his KD2B-tree and the sphere tree with the R-tree for different queries. The KD2B-tree is a paged version of the KD2-tree, which in turn is a variant of the BSP-tree. The two structures differ in two aspects: each interior node stores two iso-oriented lines to allow for overlap and gaps, and the corresponding partition lines do not clip; that is, an object is handled as a unit. The KD2B-tree outperforms the R-tree for all queries, whereas the sphere tree is inferior to the R-tree.

Hoel and Samet [1992] compare the performance of the PMR-quadtree [Nelson and Samet 1987], the R*-tree, and the $\mathrm{R}^{+}$-tree for indexing line segments. The $\mathrm{R}^{+}$-tree shows the best insertion performance, whereas the $\mathrm{R}^{*}$-tree occupies the least space. However, the insertion behavior of the $\mathrm{R}^{+}$-tree heavily depends on the page size, unlike the PMRquadtree. The performance of all structures compared is about the same, even though the PMR-quadtree shows some slight performance benefits. Although the $\mathrm{R}^{*}$-tree is more compact than the other structures, its search performance is not as good as that of the $\mathrm{R}^{+}$-tree for line segments. Unfortunately, Hoel and Samet do not report the overall performance times for the different queries.

Peloux et al. [1994] carried out a similar performance comparison of two quadtree variants, a variant of the $\mathrm{R}^{+}$. tree, and the $\mathrm{R}^{*}$-tree. What makes their study different is that all structures have been implemented on top of a commercial object-oriented system using the application programmer interface. A further difference to Hoel and Samet [1992] is that Peloux et al. used polygons rather than line segments as test data. Furthermore, they report the various times for index traversal, loading polygons, and the like. Besides showing that the $\mathrm{R}^{+}$-tree and a quadtree variant based on Hierarchical EXCELL [Tamminen 1983] outperform the $R^{*}$-tree for point queries, they clearly demonstrate that the database system must provide some means for physical clustering. Otherwise, reading a single index page may induce several page faults.

Smith and Gao [1990] compare the performance of a variant of the $\mathrm{zkdB}^{+}$tree, the grid file, the R-tree, and the $\mathrm{R}^{+}$-tree for insertions, deletions, and search operations. They also measured storage utilization. The conclusion of their experiments is that z-ordering and the grid file perform well for insertions and deletions but deliver poor search performance. $\mathrm{R}$ - and $\mathrm{R}^{+}$-trees, in contrast, offer moderate insertion and deletion performance but superior search performance. Although the $\mathrm{R}^{+}$-tree performs slightly better than the R-tree for search operations, the authors conclude that the $\mathrm{R}^{+}$-tree is not a good choice for general-purpose applications, due to its potentially poor space utilization.

Hutflesz et al. [1990] showed that the R-file has a 10 to $20 \%$ performance advantage over the R-tree on a data set containing 48,000 rectangles with a high degree of overlap (each point in the 
database was covered by 5.78 rectangles on the average).

Further experimental studies on the R-tree and related structures can be found in Frank and Barrera [1989], Kamel and Faloutsos [1992], and Kolovson and Stonebraker [1991].

Since the splitting of data buckets is an important operation in many structures, Henrich and Six [1991] studied several split strategies. Their theoretical analysis is verified by means of the LSD-tree. They also provide some performance results for the R-tree that uses their splitting strategy in comparison to the otherwise unchanged R-tree. An empirical performance comparison of the R-tree with an improved variant of z-hashing, called layered z-hashing or Iz-hashing [Hutflesz et al. 1988a], can be found in Hutflesz et al. [1991]. The proposed structure needs significantly less seek operations than the R-tree; average storage utilization is higher.

J agadish [1990a] studies the properties of different space-filling curves (zordering, Gray-coding, and Hilbertcurve). By means of theoretical considerations as well as by experimental tests, he concludes that the Hilbert mapping from multidimensional space to a line is superior to other spacefilling curves. These results are in accordance with those of Abel and Mark [1990].

When trying to summarize all those experimental comparisons, the following multidimensional access methods seem to be among the best-performing ones (in alphabetical order):

- buddy (hash) tree [Seeger and Kriegel 1990],

-cell tree with oversize shelves [Günther and Gaede 1997],

-Hilbert R-tree [Kamel and Faloutsos 1994],

-KD2B-tree [Oosterom 1990],

-PMR-quadtree [Nelson and Samet 1987],

$-\mathrm{R}^{+}$-tree [Sellis et al. 1987], and

- $\mathrm{R}^{*}$-tree [Beckmann et al. 1990]
It cannot be emphasized enough, however, that any such ranking needs to be used with great care. Clever programming can often make up for inherent deficiencies of an access method and vice versa. Other factors of unpredictable impact include the hardware used, the buffer size/page size, and the data sets. Note also that our list does not take into account access methods for which no comparative analyses have been published.

As the preceding discussion shows, although numerous experimental studies exist, they are hardly comparable. Theoretical studies may bring some more objectivity to this discussion. The problem with such studies is that they are usually very hard to perform if one wants to stick to realistic modeling assumptions. For that reason there are only a few theoretical results on the comparison of multidimensional access methods.

Regnier [1985] and Becker [1992] investigated the grid file and some of its variants. The most complete theoretical analysis of range trees can be found in Overmars et al. [1990] and Smid and Overmars [1990]. Günther and Gaede [1997] present a theoretical analysis of the cell tree. Recent analyses show that the theory of fractals seems to be particularly suitable for modeling the behavior of SAMs if the data distribution is nonuniform. ${ }^{3}$

Some more analytical work exists on the R-tree and related methods. A comparison of the $\mathrm{R}$-tree and the $\mathrm{R}^{+}$-tree has been published by Faloutsos et al. [1987]. Recently, Pagel et al. [1993a] presented an interesting probabilistic model of window query performance for the comparison of different access methods independent of implementation details. Among other things, their model reveals the importance of the perimeter as a criterion for node splitting, which has been intuitively anticipated by the

\footnotetext{
${ }^{3}$ See Faloutsos and Kamel [1994], Belussi and Faloutsos [1995], Faloutsos and Gaede [1996], and Papadopoulos and Manolopoulos [1997].
} 
inventors of the $\mathrm{R}$ *-tree [Beckmann et al. 1990]. The central formula of Pagel et al. [1993] to compute the number of disk accesses in an R-tree has been found independently by Kamel and $\mathrm{Fa}$ loutsos [1993]. Faloutsos and Kamel [1994] later refined this formula by using properties of the data set. More recently, Theodoridis and Sellis [1996] proposed a theoretical model to determine the number of disk accesses in an R-tree that requires only two parameters: the amount of data and the density in the data space. Their model also extends to nonuniform distributions.

In pursuit of an implementation-independent comparison criterion for access methods, Pagel et al. [1995] suggest using the degree of clustering. As a lower bound they assume the optimal clustering of the static situation, that is, if the complete data set has been exposed beforehand. Incidentally, the significance of clustering for access methods has been demonstrated in numerous empirical investigations as well. ${ }^{4}$

In the area of constraint database systems (see Gaede and Wallace [1997] for a recent survey) a number of interesting papers related to multidimensional access methods have been published. Kanellakis et al. [1993], for example, presented a semidynamic structure that guarantees certain worst-case bounds for space, search, and insertion. Subramanian and Ramaswamy [1995] and Hellerstein et al. [1997] complement this work by proving some important lower and upper bounds. Sexton [1997] and Stuckey [1997] look at indexing from a language point of view. Their work can be regarded as a generalization of work by Hellerstein et al. [1995], who proposed a generic framework for modeling hierarchical access methods.

\section{CONCLUSIONS}

Research in spatial database systems has resulted in a multitude of spatial

\footnotetext{
${ }^{4}$ See J agadish [1990a], Kamel and Faloutsos [1993], Brinkhoff and Kriegel [1994], Kumar [1994b], and Ng and Han [1994].
}

access methods. Even for experts it becomes more and more difficult to recognize their merits and weaknesses, since every new method seems to claim superiority to at least one access method previously published. This survey did not try to resolve this problem but rather to give an overview of the pros and cons of a variety of structures. It will come as no surprise to the reader that at present no access method has proven itself superior to all its competitors in whatever sense. Even if one benchmark declares one structure as the clear winner, another benchmark may prove the same structure inferior.

But why are such comparisons so difficult? Because there are so many different criteria to define optimality, and so many parameters that determine performance. Both time and space efficiency of an access method strongly depend on the data processed and the queries asked. An access method that performs reasonably well for iso-oriented rectangles may fail for arbitrarily oriented lines. Strongly correlated data may render an otherwise fast access method irrelevant for any practical application. An index that has been optimized for point queries may be highly inefficient for arbitrary region queries. Large numbers of insertions and deletions may deteriorate a structure that is efficient in a more static environment.

The initiative of Kriegel et al. [1990] to set up a standardized testbed for benchmarking and comparing access methods under different conditions was an important step in the right direction. The world wide web provides a convenient infrastructure to access and distribute such benchmarks [Günther et al. 1988]. Nevertheless, it remains far from easy to compare or rank different access methods. Experimental benchmarks need to be studied with care and can only be a first indicator for usability.

When it comes to technology transfer, that is, to the use of access methods in commercial products, most vendors resort to structures that are easy to un- 
derstand and implement. Quadtrees in SICAD [Siemens Nixdorf I nformationssysteme AG 1997] and Smallworld GIS [Newell and Doe 1997], R-trees in Informix [I nformix Inc. 1997], and Z-ordering in Oracle [Oracle Inc. 1995] are typical examples. Performance seems to be of minor importance in the selection, which comes as no surprise given the relatively small differences among methods in virtually all published analyses. Rather, the tendency is to take a structure that is simple and robust and to optimize its performance by a highly tuned implementation and tight integration with other system components.

Nevertheless, the implementation and experimental evaluation of access methods is essential as it often reveals deficiencies and problems that are not obvious from the design or a theoretical model. In order to make such comparative evaluations both easier to perform and easier to verify, it is essential to provide platform-independent access to the implementations of a broad variety of access methods. Some extensions of the World Wide Web, including our own MMM project [Günther et al. 1997], may provide the right technological base for such a paradigm change. Once every published paper includes a URL (uniform resource locator), that is, an Internet address that points to an implementation, possibly with a standardized user interface, transparency will increase substantially. Until then, most users will have to rely on general wisdom and their own experiments to select an access method that provides the best fit for their current application.

\section{ACKNOWLEDGMENTS}

While working on this survey, we had the pleasure of discussions with many colleagues. Special thanks go to D. Abel, A. Buchmann, C. Faloutsos, A. Frank, M. Freeston, J. C. Freytag, J. Hellerstein, C. Kolovson, H.-P. Kriegel, J . Nievergelt, J . Orenstein, P. Picouet, W.-F. Riekert, D. Rotem, J .-M. Saglio, B. Salzberg, H. Samet, M. Schiwietz, R. Schneider, M. Scholl, B. Seeger, T. Sellis, A. P. Sexton, and $\mathrm{P}$. Widmayer. We would also like to thank the referees for their detailed and insightful comments.

\section{REFERENCES}

ABEL, D. J . AND MARK, D. M. 1990. A comparative analysis of some two-dimensional orderings. Int. J . Geograph. Inf. Syst. 4, 1, 21-31.

ABEL, D. J. AND SMITH, J . L. 1983. A data structure and algorithm based on a linear key for a rectangle retrieval problem. Comput. Vis. 24, $1-13$.

ANG, C. AND TAN, T. 1997. New linear node splitting algorithm for R-trees. In Advances in Spatial Databases, M. Scholl and A. Voisard, Eds., LNCS, Springer-Verlag, Berlin/Heidelberg/New York.

Aref, W. G. And SAmet, H. 1994. The spatial filter revisited. In Proceedings of the Sixth International Symposium on Spatial Data Handling, 190-208.

BAYER, R. 1996. The universal B-tree for multidimensional indexing. Tech. Rep. I9639, Technische Universität München, Munich, Germany. http://www.leo.org/pub/comp/doc/ techreports/tum/informatik/report/1996/TUM I 9639.ps.gz.

BAyER, R. AND MCCREIGHT, E. M. 1972. Organization and maintenance of large ordered indices. Acta Inf. 1, 3, 173-189.

BAYER, R. AND SCHKOLNICK, M. 1977. Concurrency of operations on B-trees. Acta Inf. 9, 1-21.

Becker, B., Franciosa, P., Gschwind, S., Ohler, T., Thiem, F., AND Widmayer, P. 1992. Enclosing many boxes by an optimal pair of boxes. In Proceedings of STACS'92, A. Finkel and M. J antzen, Eds., LNCS 525, SpringerVerlag, Berlin/Heidel berg/New York, 475- 486.

BECKER, L. 1992. A new algorithm and a cost model for join processing with the grid file. Ph.D. thesis, Universität-Gesamthochschule Siegen, Germany.

BeckmanN, N., Kriegel, H.-P., Schneider, R., AND SEEgER, B. 1990. The R*-tree: An efficient and robust access method for points and rectangles. In Proceedings of ACM SIGMOD International Conference on Management of Data, 322-331.

Belussi, A. AND Faloutsos, C. 1995. Estimating the selectivity of spatial queries using the 'correlation' fractal dimension. In Proceedings of the 21st International Conference on Very Large Data Bases, 299-310.

BentLEY, J. L. 1975. Multidimensional binary search trees used for associative searching. Commun. ACM 18, 9, 509-517.

Bentley, J. L. 1979. Multidimensional binary search in database applications. IEEE Trans. Softw. Eng. 4, 5, 333-340.

Bentley, J. L. and Friedman, J. H. 1979. Data 
structures for range searching. ACM Comput. Surv. 11, 4, 397- 409.

Berchtold, S., Keim, D., and Kriegel, H.-P. 1996. The X-tree: An index structure for high-dimensional data. In Proceedings of the 22nd International Conference on Very Large Data Bases, (Bombay) 28-39.

Blanken, H., IJbema, A., Meek, P., And van den AKKER, B. 1990. The generalized grid file: Description and performance aspects. In Proceedings of the Sixth IEEE International Conference on Data Engineering, 380-388.

BRINKHOFF, T. 1994. Der spatial join in geodatenbanksystemen. Ph.D. Thesis, LudwigMaximilians-Universität München. Germany (in German).

BRINKHOFF, T. AND KRIEgEL, H.-P. 1994. The impact of global clustering on spatial database systems. In Proceedings of the Twentieth International Conference on Very Large Data Bases, 168-179.

Brinkhoff, T., Kriegel, H.-P., And Schneider, R. 1993a. Comparison of approximations of complex objects used for approximation-based query processing in spatial database systems. In Proceedings of the Ninth IEEE International Conference on Data Engineering, 4049.

Brinkhoff, T., KRiegel, H.-P., AND Seeger, B. 1993b. Efficient processing of spatial joins using R-trees. In Proceedings of ACM SIGMOD International Conference on Management of Data, 237-246.

BrinkhofF, T., KRIEGel, H.-P., Schneider, R., AND SEEGER, B. 1994. Multi-step processing of spatial joins. In Proceedings of the ACM SIGMOD International Conference on Management of Data, 197-208.

Brodsky, A., LASSEZ, C., LASSEZ, J .-L., AND MAHER, M.J. 1995. Separability of polyhedra for optimal filtering of spatial and constraint data. In Proceedings of the Fourteenth ACM SIGACT-SIGMOD-SIGART Symposium on Principles of Database Systems (San J ose, CA), 54- 64.

BURKHARD, W. 1984. Index maintenance for non-uniform record distributions. In Proceedings of the Third ACM SIGACT-SIGMOD Symposium on Principles of Database Systems, 173-180.

BURKHARD, W. A. 1983. Interpolation-based index maintenance. BIT 23, 274-294.

Chen, L., Drach, R., Keating, M., Louis, S., Rotem, D., And Shoshani, A. 1995. Access to multidimensional datasets on tertiary storage systems. Inf. Syst. 20, 2, 155- 183.

Comer, D. 1979. The ubiquitous B-tree. ACM Comput. Surv. 11, 2, 121-138.

Dandamudi, S. P. And Sorenson, P. G. 1986. Algorithms for BD-trees. Softw. Pract. Exper. 16, 2, 1077-1096.
Dandamudi, S. P. And Sorenson, P. G. 1991. I mproved partial-match search algorithms for BD-trees. Comput. J . 34, 5, 415- 422.

Egenhofer, M. 1989. Spatial query languages. Ph.D. Thesis, University of Maine, Orono, ME.

Egenhofer, M. 1994. Spatial SQL: A query and presentation language. IEEE Trans. Knowl. Data Eng. 6, 1, 86-95.

EvangelidIs, G. 1994. The hB ${ }^{\Pi}$-tree: A concurrent and recoverable multi-attribute index structure. Ph.D. Thesis, N ortheastern U niversity, Boston, MA.

Evangelidis, G., Lomet, D., and Salzberg, B. 1995. The $\mathrm{hB}^{\Pi}$-tree: A modified hB-tree supporting concurrency, recovery and node consolidation. In Proceedings of the 21st International Conference on Very Large Data Bases, 551-561.

Fagin, R., Nievergelt, J., Pippenger, N., AND Strong, R. 1979. Extendible hashing: A fast access method for dynamic files. ACM Trans. Database Syst. 4, 3, 315-344.

Faloutsos, C. 1986. Multiattribute hashing using Gray-codes. In Proceedings of the ACM SIGMOD International Conference on Management of Data, 227-238.

Faloutsos, C. 1988. Gray-codes for partial match and range queries. IEEE Trans. Softw. Eng. 14, 1381-1393.

Faloutsos, C. And Gaede, V. 1996. Analysis of n-dimensional quadtrees using the Hausdorff fractal dimension. In Proceedings of the 22nd International Conference on Very Large Data Bases, (Bombay), 40-50.

Faloutsos, C. And Kamel, I. 1994. Beyond uniformity and independence: Analysis of R-trees using the concept of fractal dimension. In Proceedings of the Thirteenth ACM SIGACTSIGMOD-SIGART Symposium on Principles of Database Systems, 4-13.

Faloutsos, C. And Rong, Y. 1991. DOT: A spatial access method using fractals. In Proceedings of the Seventh IEEE International Conference on Data Engineering, 152-159.

Faloutsos, C. And Roseman, S. 1989. Fractals for secondary key retrieval. In Proceedings of the Eighth ACM SIGACT-SIGMOD-SIGART Symposium on Principles of Database Systems, 247-252.

Faloutsos, C., Sellis, T., AND Roussopoulos, N. 1987. Analysis of object-oriented spatial access methods. In Proceedings of the ACM SIGMOD International Conference on Management of Data, 426-439.

Finkel, R. AND Bentley, J. L. 1974. Quad trees: A data structure for retrieval of composite keys. Acta Inf. 4, 1, 1-9.

FLAJ OLET, P. 1983. On the performance evaluation of extendible hashing and trie searching. Acta Inf. 20, 345-369. 
Frank, A. AND BARRERA, R. 1989. The fieldtree: A data structure for geographic information systems. In Design and Implementation of Large Spatial Database Systems, A. Buchmann, O. Günther, T.R. Smith, and Y.-F. Wang, Eds., LNCS 409, Springer-Verlag, Berlin/Heidel berg/New York, 29- 44.

Freeston, M. 1987. The BANG file: A new kind of grid file. In Proceedings of the ACM SIGMOD International Conference on Manage ment of Data,, 260-269.

FreEston, M. 1989a. Advances in the design of the BANG file. In Proceedings of the Third International Conference on Foundations of Data Organization and Algorithms, LNCS 367, Springer-Verlag, Berlin/Heidelberg/New York, 322-338.

FreEston, M. 1989b. A well-behaved structure for the storage of geometric objects. In Design and Implementation of Large Spatial Database Systems, A. Buchmann, O. Günther, T. R. Smith, and Y.-F. Wang, Eds., LNCS 409, Springer-Verlag, Berlin/Heidelberg/New York, 287-300.

FREESTON, M. 1995. A general solution of the n-dimensional B-tree problem. In Proceedings of the ACM SIGMOD International Conference on Management of Data, 80-91.

FreEston, M. 1997. On the complexity of BVtree updates. In Proceedings of CDB'97 and CP'96 Workshop on Constraint Databases and their Application, V. Gaede, A. Brodsky, O. Günther, D. Srivastava, V. Vianu, and M. Wallace, Eds., LNCS 1191, Springer-Verlag, Berlin/Heidel berg/New York, 282-293.

Fuchs, H., Abram, G. D., and Grant, E.D. 1983. Near real-time shaded display of rigid objects. Computer Graph. 17, 3, 65-72.

FuCHS, H., KEDEM, Z., AND NAYLOR, B. 1980. On visible surface generation by a priori tree structures. Computer Graph. 14, 3.

GAEDE, V. 1995a. Geometric information makes spatial query processing more efficient. In Proceedings of the Third ACM International Workshop on Advances in Geographic Information Systems (ACM-GIS'95) (Baltimore, MD) 45-52.

GAEDE, V. 1995b. Optimal redundancy in spatial database systems. In Advances in Spatial Databases, M. J . E genhofer and J . R. Herring, Eds., LNCS 951, Springer-Verlag, Berlin/Heidel berg/New York, 96-116.

GAede, V. AND RIEKERT, W.-F. 1994. Spatial access methods and query processing in the object-oriented GIS GODOT. In Proceedings of the AGDM'94 Workshop (Delft, The Netherlands), Netherlands Geodetic Commission, 40-52.

Gaede, V. AND Wallace, M. 1997. An informal introduction to constraint databases. In Proceedings of CDB'97 and CP'96 Workshop on Constraint Databases and their Application,
V. Gaede, A. Brodsky, O. Günther, D. Srivastava, V. Vianu, and M. Wallace, Eds., LNCS 1191, Springer-Verlag, Berlin/Heidelberg/ New York, 7-52.

Garg, A. K. And Gotlieb, C. C. 1986. Orderpreserving key transformation. ACM Trans. Database Syst. 11, 2, 213-234.

GreEnE, D. 1989. An implementation and performance analysis of spatial data access methods. In Proceedings of the Fifth IEEE International Conference on Data Engineering, 606-615.

GüNTHER, O. 1988. Efficient Structures for Geometric Data Management. LNCS 337, Springer-Verlag, Berlin/Heidelberg/N ew York.

GüNTHER, O. 1989. The cell tree: An object-oriented index structure for geometric databases. In Proceedings of the Fifth IEEE International Conference on Data Engineering, 598- 605.

GüNTHER, O. 1991. Evaluation of spatial access methods with oversize shelves. In Geographic Database Management Systems, G. Gambosi, M. Scholl, and H.-W. Six, Eds., Springer-Verlag, Berlin/Heidel berg/New Y ork, 177-193.

GüNTHER, O. 1993. Efficient computation of spatial joins. In Proceedings of the Ninth IEEE International Conference on Data Engineering, 50-59.

Günther, O. AND BILmes, J. 1991. Tree-based access methods for spatial databases: Implementation and performance evaluation. IEEE Trans. Knowl. Data Eng. 3, 3, 342-356.

Günther, O. ANd BuchmanN, A. 1990. Research issues in spatial databases. SIGMOD Rec. 19, 4, 61- 68

Günther, O. AND Gaede, V. 1997. Oversize shelves: A storage management technique for large spatial data objects. Int. J. Geog. Inf. Syst. 11, 1, 5-32.

Günther, O. And Noltemeier, H. 1991. Spatial database indices for large extended objects. In Proceedings of the Seventh IEEE International Conference on Data Engineering, 520526.

Günther, O., Müller, R., Schmidt, P., Bhargava, H., AND KRISHNAN, R. 1997. MMM: A WWW-based approach for sharing statistical software modules. IEEE I nternet Comput. 1, 3.

Günther, O., Oria, V., Picouet, P., Saglio, J.-M., AND SCHOLL, M. 1998. Benchmarking spatial joins à la carte. In Proceedings of the 10th International Conference on Scientific and Statistical Database Management. IEEE, New York.

GüTING, R. H. 1989. Gral: An extendible relational database system for geometric applications. In Proceedings of the Fifteenth I nternational Conference on Very Large Data Bases, 33- 44.

Güting, R. H. ANd Schneider, M. 1993. 
Realms: A foundation for spatial data types in database systems. In Advances in Spatial Databases, D. Abel and B. C. Ooi, Eds., LNCS 692, Springer-Verlag, Berlin/Heidelberg/New York.

Guttman, A. 1984. R-trees: A dynamic index structure for spatial searching. In Proceedings of the ACM SIGMOD International Conference on Management of Data, 47-54.

Hellerstein, J. M., Koutsoupias, E., And PapadIMITRIOU, C. H. 1997. Towards a theory of indexability. In Proceedings of the Sixteenth ACM SIGACT-SIGMOD-SIGART Symposium on Principles of Database Systems.

Hellerstein, J. M., Naughton, J. F., and PfefFER, A. 1995. Generalized search trees for database systems. In Proceedings of the 21st International Conference on Very Large Data Bases, 562-573.

HENRICH, A. 1995. Adapting the transformation technique to maintain multidimensional nonpoint objects in k-d-tree based access structures. In Proceedings of the Third ACM International Workshop on Advances in Geographic Information Systems (ACM-GIS'95) (Baltimore, MD) ACM Press, N ew York.

Henrich, A. AND Möller, J. 1995. Extending a spatial access structure to support additional standard attributes. In Advances in Spatial Databases, M. J . Egenhofer and J . R. Herring, Eds., LNCS 951, Springer-Verlag, Berlin/Hei del berg/New York, 132-151.

Henrich, A. And Six, H.-W. 1991. How to split buckets in spatial data structures. In Geographic Database Management Systems, G. Gambosi, M. Scholl, and H.-W. Six, Eds., Springer-Verlag, Berlin/Heidelberg/New York, 212-244.

HENRICH, A., SIX, H.-W., AND WIDMAYER, P. 1989. The LSD tree: Spatial access to multidimensional point and non-point objects. In Proceedings of the Fifteenth International Conference on Very Large Data Bases, 45-53.

HINRICHS, K. 1985. I mplementation of the grid file: Design concepts and experience. BIT 25, 569-592.

Hoel, E. G. and Samet, H. 1992. A qualitative comparison study of data structures for large segment databases. In Proceedings of the ACM SIGMOD International Conference on Management of Data, 205-214.

Hoel, E. G. And SAmet, H. 1995. Benchmarking spatial join operations with spatial output. In Proceedings of the 21st International Conference on Very Large Data Bases, 606618.

Hutflesz, A., Six, H.-W., And Widmayer, P. 1988a. Globally order preserving multidimensional linear hashing. In Proceedings of the Fourth IEEE International Conference on Data Engineering, 572-579.

Hutflesz, A., Six, H.-W., And Widmayer, P. 1988b. Twin grid files: Space optimizing access schemes. In Proceedings of the ACM SIGMOD International Conference on Management of Data, 183-190.

Hutflesz, A., Six, H.-W., and Widmayer, P. 1990. The R-file: An efficient access structure for proximity queries. In Proceedings of the Sixth IEEE International Conference on Data Engineering, 372-379.

Hutflesz, A., Widmayer, P., and Zimmermann, C. 1991. Global order makes spatial access faster. In Geographic Database Management Systems, G. Gambosi, M. Scholl, and H.-W. Six, Eds., Springer-Verlag, Berlin/Hei del berg/ New York, 161-176.

INFORMIX INC. 1997. The DataBlade architecture. URL http://www.informix.com.

J AGADISH, H. V. 1990a. Linear clustering of objects with multiple attributes. In Proceedings of the ACM SIGMOD International Conference on Management of Data, 332-342.

J AGADISH, H. V. 1990b. On indexing line segments. In Proceedings of the Sixteenth International Conference on Very Large Data Bases, 614-625.

J AGADISH, H. V. 1990c. Spatial search with polyhedra. In Proceedings of the Sixth IEEE International Conference on Data Engineering, 311-319.

Kamel, I. And Faloutsos, C. 1992. Parallel Rtrees. In Proceedings of the ACM SIGMOD International Conference on Management of Data, 195-204.

Kamel, I. And Faloutsos, C. 1993. On packing R-trees. In Proceedings of the Second International Conference on Information and Knowledge Management, 490- 499.

Kamel, I. And Faloutsos, C. 1994. Hilbert Rtree: An improved R-tree using fractals. In Proceedings of the Twentieth International Conference on Very Large Data Bases, 500 509.

Kamel, I., Khalil, M., and Kouramajian, V. 1996. Bulk insertion in dynamic R-trees. In Proceedings of the Seventh International Symposium on Spatial Data Handling (Delft, The Netherlands), 3B.31-3B.42.

Kanellakis, P. C., Ramaswamy, S., Vengroff, D. E., AND VITTER, J.S. 1993. I ndexing for data models with constraints and classes. In Proceedings of the Twelfth ACM SIGACTSIGMOD-SIGART Symposium on Principles of Database Systems, 233-243.

Kedem, G. 1982. The quad-CIF tree: A data structure for hierarchical on-line algorithms. In Proceedings of the Nineteenth Conference on Design and Automation, 352-357.

Kemper, A. And Wallrath, M. 1987. An analysis of geometric modeling in database systems. ACM Comput. Surv. 19, 1, 47-91.

KLINGER, A. 1971. Pattern and search statis- 
tics. In Optimizing Methods in Statistics, S. Rustagi, Ed., 303-337.

Knott, G. 1975. Hashing functions. Comput. J . $18,3,265-278$.

Kolovson, C. 1990. Indexing techniques for multi-dimensional spatial data and historical data in database management systems. Ph.D. Thesis, University of California at Berkeley.

Kolovson, C. and Stonebraker, M. 1991. Segment indexes: Dynamic indexing techniques for multi-dimensional interval data. In Proceedings of the ACM SIGMOD International Conference on Management of Data, 138-147.

KRIEGEL, H.-P. 1984. Performance comparison of index structures for multikey retrieval. In Proceedings of the ACM SIGMOD International Conference on Management of Data, 186-196.

Kriegel, H.-P., Heep, P., Heep, S., Schiwietz, M., AND SCHNEIDER, R. 1991. An access method based query processor for spatial database systems. In Geographic Database Manage ment Systems, G. Gambosi, M. Scholl, and H.-W. Six, Eds., Springer-Verlag, Berlin/Heidel berg/New York, 273-292.

Kriegel, H.-P., SchiWIETZ, M., SCHNeIder, R., AND SeEger, B. 1990. Performance comparison of point and spatial access methods. In Design and Implementation of Large Spatial Database Systems, A. Buchmann, O. Günther, T. R. Smith, and Y.-F. Wang, Eds., LNCS 409, Springer-Verlag, Berlin/Heidel berg/New York, 89-114.

Kriegel, H.-P. And Seeger, B. 1986. Multidimensional order preserving linear hashing with partial expansions. In Proceedings of the International Conference on Database Theory, LNCS 243, Springer-Verlag, Berlin/Heidelberg/New York.

Kriegel, H.-P. AND Seeger, B. 1987. Multidimensional quantile hashing is very efficient for non-uniform record distributions. In Proceedings of the Third IEEE International Conference on Data Engineering, 10-17.

Kriegel, H.-P. ANd Seeger, B. 1988. PlOPhashing: A grid file without directory. In Proceedings of the Fourth IEEE International Conference on Data Engineering, 369-376.

KRIEgel, H.-P. AND SEeger, B. 1989. Multidimensional quantile hashing is very efficient for non-uniform distributions. Inf. Sci. 48, 99- 117.

KORNACKER, M. AND BANKS, D. 1995. High-concurrency locking in R-trees. In Proceedings of the 21st International Conference on Very Large Data Bases, 134-145.

KUMAR, A. 1994a. G-tree: A new data structure for organizing multidimensional data. IEEE Trans. Knowl. Data Eng. 6, 2, 341-347.

KUMAR, A. 1994b. A study of spatial clustering techniques. In Proceedings of the Fifth Confer- ence on Database and Expert Systems Applications (DEXA'94), D. Karagiannis, Ed., LNCS 856, Springer-Verlag, Berlin/Heidelberg/New York, 57-70.

LARSON, P. A. 1980. Linear hashing with partial expansions. In Proceedings of the Sixth International Conference on Very Large Data Bases, 224-232.

LEHMAN, P. AND YAO, S. 1981. Efficient locking for concurrent operations on B-trees. ACM Trans. Database Syst. 6, 4, 650-670.

LIN, K.-I., JagADISH, H., AND Faloutsos, C. 1994. The TV-tree: An index structure for high-dimensional data. VLDB J. 3, 4, 517543.

LITWIN, W. 1980. Linear hashing: A new tool for file and table addressing. In Proceedings of the Sixth International Conference on Very Large Data Bases, 212-223.

Lo, M. AND RAVISHANKAR, C. 1994. Spatial joins using seeded trees. In Proceedings of the ACM SIGMOD International Conference on Management of Data, 209-220.

LOMET, D. B. 1983. Boundex index exponential hashing. ACM Trans. Database Syst. 8, 1, $136-165$.

LOMET, D. B. 1991. Grow and post index trees: Role, techniques and future potential. In Advances in Spatial Databases, O. Günther and H. Schek, Eds., LNCS 525, Springer-Verlag, Berlin/Heidel berg/New York, 183-206.

Lomet, D. B. and Salzberg, B. 1989. The hBtree: A robust multiattribute search structure. In Proceedings of the Fifth IEEE International Conference on Data Engineering, 296-304.

Lomet, D. B. and Salzberg, B. 1990. The hBtree: A multiattribute indexing method with good guaranteed performance. ACM Trans. Database Syst. 15, 4, 625-658. Reprinted in Readings in Database Systems, $M$. Stonebraker, Ed., Morgan-Kaufmann, San Mateo, CA, 1994.

Lomet, D. B. And SAlzberg, B. 1992. Access method concurrency with recovery. In Proceedings of the ACM SIGMOD International Conference on Management of Data, 351-360.

LU, H. AND OOI, B.-C. 1993. Spatial indexing: Past and future. IEEE Data Eng. Bull. 16, 3, 16-21.

Matsuyama, T., Hao, L. V., and Nagao, M. 1984. A file organization for geographic information systems based on spatial proximity. Int. J . Comput. Vis. Graph. I mage Process. 26, 3, 303-318.

MORTON, G. 1966. A computer oriented geodetic data base and a new technique in file se quencing. IBM Ltd.

Nelson, R. and Samet, H. 1987. A population analysis for hierarchical data structures. In Proceedings of the ACM SIGMOD Interna- 
tional Conference on Management of Data, 270-277.

Newell, R. G. And Doe, M. 1997. Discrete ge ometry with seamless topology in a GIS. URL http://www.smallworld-us.com.

NG, R. T. AND HAN, J. 1994. Efficient and effective clustering methods for spatial data mining. In Proceedings of the Twentieth International Conference on Very Large Data Bases, 144-154.

NG, V. AND KamedA, T. 1993. Concurrent accesses to R-trees. In Advances in Spatial Databases, D. Abel and B. C. Ooi, Eds., LNCS 692, Springer-Verlag, Berlin/Heidelberg/New York, 142-161.

NG, V. And Kameda, T. 1994. The R-link tree: A recoverable index structure for spatial data. In Proceedings of the Fifth Conference on Database and Expert Systems Applications (DEXA'94), D. Karagiannis, Ed., LNCS 856, Springer-Verlag, Berlin/Heidelberg/New York, 163-172.

Nievergelt, J . 1989. 7 \pm 2 criteria for assessing and comparing spatial data structures. In De sign and I mplementation of Large Spatial Database Systems, A. Buchmann, O. Günther, T. R. Smith, and Y.-F. Wang, Eds., LNCS 409, Springer-Verlag, Berlin/Heidel berg/New York, 3-27.

Nievergelt, J. and Hinrichs, K. 1987. Storage and access structures for geometric data bases. In Proceedings of the International Conference on Foundations of Data Organization, S. Ghosh, Y. Kambayashi, and K. Tanaka, Eds., Plenum, New York.

Nievergelt, J ., Hinterberger, H., And Sevcik, K. 1981. The grid file: An adaptable, symmetric multikey file structure. In Proceedings of the Third $\mathrm{ECl}$ Conference, $\mathrm{A}$. Duijvestijn and $\mathrm{P}$. Lockemann, Eds., LNCS 123, Springer-Verlag, Berlin/Heidel berg/New York, 236-251.

Nievergelt, J., Hinterberger, H., And Sevcik, K.C. 1984. The grid file: An adaptable, symmetric multikey file structure. ACM Trans. Database Syst. 9, 1, 38-71.

Ohsawa, Y. ANd SaKauchi, M. 1983. BD-tree: A new $n$-dimensional data structure with efficient dynamic characteristics. In Proceedings of the Ninth World Computer Congress, IFIP 1983, 539-544.

Ohsawa, Y. and Sakauchi, M. 1990. A new tree type data structure with homogeneous node suitable for a very large spatial database. In Proceedings of the Sixth IEEE International Conference on Data Engineering, 296-303.

OoI, B. C. 1990. Efficient Query Processing in Geographic Information Systems. LNCS 471, Springer-Verlag, Berlin/Heidel berg/New York.

OoI, B. C., McDonell, K. J., And Sacks-Davis, R. 1987. Spatial kd-tree: An indexing mechanism for spatial databases. In Proceed- ings of the IEEE Computer Software and Applications Conference, 433- 438.

OoI, B. C., SAcks-Davis, R., AND McDonelL, K.J. 1991. Spatial indexing by binary decomposition and spatial bounding. Inf. Syst. J. 16, 2, 211-237.

Oosterom, P. 1990. Reactive data structures for geographic information systems. Ph.D. Thesis, University of Leiden, The Netherlands.

ORACLE INC. 1995. Oracle 7 multidimension: Advances in relational database technology for spatial data management. White paper.

ORENSTEIN, J. 1982. Multidimensional tries used for associative searching. Inf. Process. Lett. 14, 4, 150-157.

OREnSTEIN, J . 1983. A dynamic file for random and sequential accessing. In Proceedings of the Ninth International Conference on Very Large Data Bases, 132-141.

Orenstein, J. 1989a. Redundancy in spatial databases. In Proceedings of the ACM SIGMOD International Conference on Manage ment of Data, 294-305.

ORENSTEIN, J . 1989b. Strategies for optimizing the use of redundancy in spatial databases. In Design and Implementation of Large Spatial Database Systems, A. Buchmann, O. Günther, T. R. Smith, and Y.-F. Wang, Eds., LNCS 409, Springer-Verlag, Berlin/Heidel berg/New York, 115- 134.

Orenstein, J. 1990. A comparison of spatial query processing techniques for native and parameter space. In Proceedings of the ACM SIGMOD International Conference on Management of Data, 343-352.

Orenstein, J. And Merrett, T. H. 1984. A class of data structures for associative searching. In Proceedings of the Third ACM SIGACTSIGMOD Symposium on Principles of Database Systems, 181- 190.

OREnStein, J. A. 1986. Spatial query processing in an object-oriented database system. In Proceedings of the ACM SIGMOD International Conference on Management of Data, 326-333.

Oтоо, E.J. 1984. A mapping function for the directory of a multidimensional extendible hashing. In Proceedings of the Tenth International Conference on Very Large Data Bases, 493-506.

Отоо, Е. J. 1985. Symmetric dynamic index maintenance scheme. In Proceedings of the International Conference on Foundations of Data Organization, Plenum, New York, 283296.

Отоо, E. J. 1986. Balanced multidimensional extendible hash tree. In Proceedings of the Fifth ACM SIGACT-SIGMOD Symposium on Principles of Database Systems, 100-113.

OUKSEL, M. 1985. The interpolation based grid 
file. In Proceedings of the Fourth ACM SIGACT-SIGMOD Symposium on Principles of Database Systems, 20-27.

Ouksel, M. And Scheuermann, P. 1983. Storage mappings for multidimensional linear dynamic hashing. In Proceedings of the Second ACM SIGACT-SIGMOD Symposium on Principles of Database Systems, 90-105.

Ouksel, M. A. And Mayer, O. 1992. A robust and efficient spatial data structure. Acta Inf. 29, 335-373.

Overmars, M. H., Smid, M. H., Berg, T., AND VAn KREVELD, M.J. 1990. Maintaining range trees in secondary memory: Part I: Partitions. Acta Inf. 27, 423- 452.

Pagel, B. U., Six, H.-W., and Toben, H. 1993 . The transformation technique for spatial objects revisited. In Advances in Spatial Databases, D. Abel and B. C. Ooi, Eds., LNCS 692, Springer-Verlag, Berlin/Heidel berg/New York, 73- 88.

Pagel, B. U., SiX, H.-W., AND Winter, M. 1995. Window query optimal clustering of spatial objects. In Proceedings of the Fourteenth ACM SIGACT-SIGMOD-SIGART Symposium on Principles of Database Systems, 86-94.

Pagel, B. U., Six, H.-W., Toben, H., AND WidMAYER, P. 1993b. Towards an analysis of range query performance in spatial data structures. In Proceedings of the Twelfth ACM SIGACT-SIGMOD-SIGART Symposium on Principles of Database Systems, 214-221.

Papadias, D., Theodoridis, Y., Sellis, T., AND EGENHOFER, M.J. 1995. Topological relations in the world of minimum bounding rectangles: A study with R-trees. In Proceedings of the ACM SIGMOD International Conference on Management of Data, 92-103.

Papadopoulos, A. And Manolopoulos, Y. 1997. Performance of nearest neighbor queries in R-trees. In Proceedings of the International Conference on Database Theory (ICDT'97), F. Afrati and P. Kolaitis, Eds., LNCS 1186, Springer-Verlag, Berlin/Heidel berg/New York, 394- 408.

Peloux, J., Reynal, G., And Scholl, M. 1994. Evaluation of spatial indices implemented with the $\mathrm{O}_{2}$ DBMS. Ingénièrie des Systèmes d'I nformation 6.

Preparata, F. P. and Shamos, M. I. 1985. Computational Geometry. Springer-Verlag, New York.

REGNIER, M. 1985. Analysis of the grid file algorithms. BIT 25, 335-357.

RoBinson, J.T. 1981. The K-D-B-tree: A search structure for large multidimensional dynamic indexes. In Proceedings of the ACM SIGMOD International Conference on Management of Data, 10-18.

Rotem, D. 1991. Spatial join indices. In Pro- ceedings of the Seventh IEEE International Conference on Data Engineering, 10-18.

Roussopoulos, N. AND LEIFKER, D. 1984. An introduction to PSQL: A pictorial structured query language. In Proceedings of the IEEE Workshop on Visual Languages.

Roussopoulos, N. AND LEIFKER, D. 1985. Direct spatial search on pictorial databases using packed R-trees. In Proceedings of the ACM SIGMOD International Conference on Management of Data, 17-31.

Sagan, H. 1994. Space-Filling Curves. Springer-Verlag, Berlin/Heidelberg/N ew York.

SAMET, H. 1984. The quadtree and related hierarchical data structure. ACM Comput. Surv. $16,2,187-260$.

SAMET, H. 1988. Hierarchical representation of collections of small rectangles. ACM Comput. Surv. 20, 4, 271-309.

SAmet, H. 1990a. Applications of Spatial Data Structures. Addison-Wesley, Reading, MA.

SAmet, H. 1990b. The Design and Analysis of Spatial Data Structures. Addison-Wesley, Reading, MA.

Samet, H. And Webber, R. E. 1985. Storing a collection of polygons using quadtrees. ACM Trans. Graph. 4, 3, 182-222.

SCHIWIETZ, M. 1993. Speicherung und anfragebearbeitung komplexer geo-objekte. Ph.D. The sis, Ludwig-Maximilians-Universität München, Germany (in German).

Schneider, R. AND KRIEgel, H.-P. 1992. The TR*-tree: A new representation of polygonal objects supporting spatial queries and operations. In Proceedings of the Seventh Workshop on Computational Geometry, LNCS 553, Springer-Verlag, Berlin/Heidelberg/New York, 249-264.

SCHOLl, M. AND VoISARd, A. 1989. Thematic map modeling. In Design and Implementation of Large Spatial Database Systems, A. Buchmann, O. Günther, T.R. Smith, and Y.-F. Wang, Eds., LNCS 409, Springer-Verlag, Berlin/Heidel berg/New York.

SeEger, B. 1991. Performance comparison of segment access methods implemented on top of the buddy-tree. In Advances in Spatial Databases, O. Günther and H. Schek, Eds., LNCS 525, Springer-Verlag, Berlin/Heidelberg/New York, 277-296.

Seeger, B. AND Kriegel, H.-P. 1988. Techniques for design and implementation of spatial access methods. In Proceedings of the Fourteenth International Conference on Very Large Data Bases, 360-371.

Seeger, B. And Kriegel, H.-P. 1990. The buddy-tree: An efficient and robust access method for spatial data base systems. In Proceedings of the Sixteenth International Conference on Very Large Data Bases, 590-601.

Sellis, T., Roussopoulos, N., and Faloutsos, C. 


\section{Multidimensional Access Methods}

1987. The $\mathrm{R}^{+}$-tree: $\mathrm{A}$ dynamic index for multi-dimensional objects. In Proceedings of the Thirteenth International Conference on Very Large Data Bases, 507-518.

Sevcik, K. And Koudas, N. 1996. Filter trees for managing spatial data over a range of size granularities. In Proceedings of the 22th International Conference on Very Large Data Bases (Bombay), 16-27.

Sexton, A. P. 1997. Querying indexed files. In Proceedings of the CDB'97 and CP'96 Workshop on Constraint Databases and Their Application, V. Gaede, A. Brodsky, O. Günther, D. Srivastava, V. Vianu, and M. Wallace, Eds., LNCS 1191, Springer-Verlag, Berlin/ Heidel berg/N ew York, 263-281.

SHEKHAR, S. AND LIU, D.-R. 1995. CCAM: A connectivity-clustered access method for aggregate queries on transportation networks: A summary of results. In Proceedings of the Eleventh IEEE International Conference on Data Engineering, 410-419.

Siemens Nixdorf Informationssysteme AG 1997. URL http://www.sni.de.

SIX, H. AND WIDMAYER, P. 1988. Spatial searching in geometric databases. In Proceedings of the Fourth IEEE International Conference on Data Engineering, 496-503.

Smid, M. H. And Overmars, M. H. 1990. Maintaining range trees in secondary memory part II: Lower bounds. Acta Inf. 27, 453- 480.

Smith, T. R. And GAo, P. 1990. Experimental performance evaluations on spatial access methods. In Proceedings of the Fourth International Symposium on Spatial Data Handling (Zürich), 991-1002.

Stonebraker, M. (Ed.) 1994. Readings in Database Systems. Morgan-Kaufmann, San Mateo, CA.

Stonebraker, M., Sellis, T., and Hanson, E. 1986. An analysis of rule indexing implementations in data base systems. In Proceed- ings of the First International Conference on Expert Data Base Systems.

StuCKey, P. 1997. Constraint search trees. In Proceedings of the International Conference on Logic Programming (CLP'97), L. Naish, Ed., MIT Press, Cambridge, MA.

Subramanian, S. And Ramaswamy, S. 1995. The P-range tree: A new data structure for range searching in secondary memory. In Proceedings of the ACM-SIAM Symposium on Discrete Algorithms (SODA'95).

TAmminen, M. 1982. The extendible cell method for closest point problems. BIT 22, 27-41.

TAMminen, M. 1983. Performance analysis of cell based geometric file organisations. Int. J . Comp. Vis. Graph. Image Process. 24, $160-$ 181.

TAmminen, M. 1984. Comment on quad- and octrees. Commun. ACM 30, 3, 204-212.

Theodoridis, Y. AND Sellis, T. K. 1996. A model for the prediction of R-tree performance. In Proceedings of the Fifteenth ACM SIGACT-SIGMOD-SIGART Symposium on Principles of Database Systems, 161-171.

Tropf, H. AND Herzog, H. 1981. Multidimensional range search in dynamically balanced trees. Angewandte Informatik 2, 71-77.

WhANG, K.-Y. AND KRISHNAMURTHY, R. 1985. Multilevel grid files. IBM Research Laboratory, Yorktown Heights, NY.

White, M. 1981. N-trees: Large ordered indexes for multi-dimensional space. Tech. Rep., Application Mathematics Research Staff, Statistical Research Division, US Bureau of the Census.

WidMAYER, P. 1991. Datenstrukturen für Geodatenbanken. In Entwicklungstendenzen bei Datenbank-Systemen, G. Vossen and K.-U. Witt, Eds., Oldenbourg-Verlag, Munich, Chapter 9, 317-361 (in German).

Received August 1995; revised August 1997; accepted J anuary 1998 